\title{
Enantioselective One-pot Three-component Synthesis of Propargylamines
}

\author{
Alakesh Bisai and Vinod K. Singh* \\ Department of Chemistry, Indian Institute of Technology, Kanpur, \\ India, 208016. \\ vinodks@iitk.ac.in
}

\section{Supporting Information}

Experimental and General Procedure for the Enantioselective three Component Synthesis of Propargylamines

Spectral data for compound (Table 1, Entry 9) and compound (Table 2, Entry 1) S4

Spectral data for compound (Table 2, Entry 1)

Spectral data for compound (Table 2, Entry 2)

Spectral data for compound (Table 2, Entry 3)

S7

Spectral data for compound (Table 2, Entry 4)

S8

Spectral data for compound (Table 2, Entry 4)

S9

Spectral data for compound (Table 2, Entry 5)

$\mathrm{S} 10$

Spectral data for compound (Table 2, Entry 6)

$\mathrm{S} 11$

Spectral data for compound (Table 2, Entry 7)

$\mathrm{S} 12$

Spectral data for compound (Table 2, Entry 8)

$\mathrm{S} 13$

Spectral data for compound (Table 2, Entry 8)

S14

Spectral data for compound (Table 2, Entry 9)

$\mathrm{S} 15$

Spectral data for compound (Table 2, Entry 9)

S16

Spectral data for compound (Table 3, Entry 2)

$\mathrm{S} 17$

Spectral data for compound (Table 3, Entry 2)

S18

Spectral data for compound (Table 3, Entry 3)

S19

Spectral data for compound (Table 3, Entry 4)

S20

Spectral data for compound (Table 4, Entry 4)

S21

Spectral data for compound (Table 3, Entry 6)

S22

Spectral data for compound (Table 3, Entry 6)

S23

Spectral data for compound (Table 5, Entry 5)

S24

Spectral data for compound (Table 5, Entry 5)

S25

Spectral data for compound (Table 5, Entry 6)

S26

Spectral data for compound (Table 4, Entry 1)

S27

Spectral data for compound (Table 4, Entry 1)

S28

Spectral data for compound (Table 4, Entry 2)

S29

Spectral data for compound (Table 4, Entry 2)

S30

Spectral data for compound (Table 4, Entry 3)

S31

Spectral data for compound (Table 4, Entry 4)

S32

Spectral data for compound (Table 4, Entry 4)

S33 
Spectral data for compound (Table 4, Entry 5) $\quad$ S34

Spectral data for compound (Table 4, Entry 5)

Spectral data for compound (Table 4, Entry 6) $\quad$ S36

$\begin{array}{ll}\text { Spectral data for compound (Table 4, Entry 6) } & \text { S37 }\end{array}$

Spectral data for compound (Table 4, Entry 7) S38

Spectral data for compound (Table 4, Entry 7) $\quad$ S39

Spectral data for compound (Table 4, Entry 8) $\quad$ S40

Spectral data for compound (Table 4, Entry 8) $\quad$ S41

Spectral data for compound (Table 4, Entry 9) $\quad$ S42

Spectral data for compound (Table 4, Entry 9) $\quad$ S43

Spectral data for compound (Table 4, Entry 10) $\quad$ S44

Spectral data for compound (Table 4, Entry 10)

Spectral data for compound (Table 4, Entry 11) S46

Spectral data for compound (Table 4, Entry 11) $\quad$ S47

Spectral data for compound (Table 4, Entry 12) $\quad$ S48

$\begin{array}{ll}\text { Spectral data for compound (Table 4, Entry 12) } & \text { S49 }\end{array}$

Spectral data for compound (Table 4, Entry 13) S50

Spectral data for compound (Table 4, Entry 14) S51

Spectral data for compound (Table 4, Entry 14) S52

Spectral data for compound (Table 5, Entry 2) S53

Spectral data for compound (Table 5, Entry 3) $\quad$ S54

Spectral data for compound (Table 5, Entry 4) $\quad$ S55

Spectral data for compound (Table 5, Entry 4) S56

$\begin{array}{ll}\text { References } & \text { S57 }\end{array}$ 


\section{Experimental}

\section{General}

All reactions were carried out under an atmosphere of nitrogen or argon in oven dried glassware with magnetic stirring. All the aldehydes, aromatic amines and terminal alkynes were purchased from Lancaster and used as received. ${ }^{1} \mathrm{H}$ and ${ }^{13} \mathrm{C}$ NMR spectra were recorded on Joel PMX (400 MHz) NMR spectrometer in $\mathrm{CDCl}_{3}$. Chemical shifts are reported in delta $(\delta)$ units, in parts per million $(\mathrm{ppm})$ downfield from tetramethylsilane. $\mathrm{CDCl}_{3}$ was used as internal standard (77.0 ppm) for ${ }^{13} \mathrm{C}$ NMR. Coupling constants were reported in Hz. Splitting patterns are designated as s for singlet; $\mathrm{d}$ for doublet; $\mathrm{t}$ for triplet; $\mathrm{q}$ for quartet; $\mathrm{m}$ for multiplet, bs for broad singlet. IR spectra were measured with Bruker FT/IR Vector 22 spectrometer. Routine monitoring of reactions were performed using precoated silicagel tlc plates from E-Merck. All the chromatographic separations were carried out by using silica gel (Acme's, 100-200 mesh). Petroleum ether used was of boiling range $60-80^{\circ} \mathrm{C}$. Melting points were determined by using Perfit apparatus and are uncorrected. The enantiomeric excess was determined by Diacel Chiralcel OD-H or Chiralpak AD-H column using iso-propanol and n-hexane as eluents at $25^{\circ} \mathrm{C}$.

\section{General Procedure for the enantioselective three component synthesis of Propargylamines catalyzed by $\mathrm{Cu}(\mathrm{I})-(S, S)-1 \mathrm{~b}$ Complex:}

A solution of a ligand $\mathbf{1 b}(0.012 \mathrm{mmol}, 6 \mathrm{~mol} \%)$ and $\mathrm{Cu}(\mathrm{I})(\mathrm{MeCN})_{4} \mathrm{PF}_{6}(0.01$ $\mathrm{mmol}, 5 \mathrm{~mol} \%)$ in dry chloroform $(2 \mathrm{~mL})$ was stirred at room temperature for $30 \mathrm{~min}$. An aldehyde $(0.2 \mathrm{mmol})$ and an aromatic amine $(0.2 \mathrm{mmol})$ were added and the whole mixture was stirred for additional $15 \mathrm{~min}$ at the same temperature. The reaction mixture was cooled to $0^{\circ} \mathrm{C}$ and an alkyne $(0.3 \mathrm{mmol})$ was added and the reaction mixtutre was allowed to warm to $20-25^{\circ} \mathrm{C}$. After completion of the reaction (monitoring by TLC), the mixture was concentrated in vacuo and purified over silica gel by column chromatography (2-10\% EtOAc in hexane) yielding pure propargylamine. 


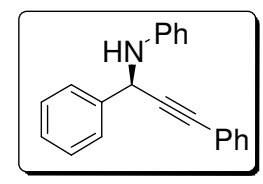

(R)-(1, 3-Diphenyl-prop-2-ynyl)-phenyl amine (Table 1, Entry 9).

$96 \%$ ee; isolated in $98 \%$ yield as colorless solid; $\mathrm{MP}=88-90^{\circ} \mathrm{C} ; \mathrm{R}_{\mathrm{f}}=0.57$ (EtOAc:

petroleum ether = 1:19); HPLC conditions: Diacel chiralcel OD-H $(4.6 \mathrm{~cm} \mathrm{I.D.} \mathrm{x} 25 \mathrm{~cm})$, 99:1 hexanes: ${ }^{i}-\mathrm{PrOH}, 0.5 \mathrm{~mL} / \mathrm{min}$ flow rate, $\lambda=254 \mathrm{~nm}, \mathrm{~T}_{\mathrm{R}}$ (major) $=24.48 \mathrm{~min}$ and $\mathrm{T}_{\mathrm{R}}$ $($ minor $)=29.52$ min; Optical Rotation: $[\alpha]^{25}+114.3\left(\mathrm{c} 2.0, \mathrm{CHCl}_{3}\right) .{ }^{1,2}$
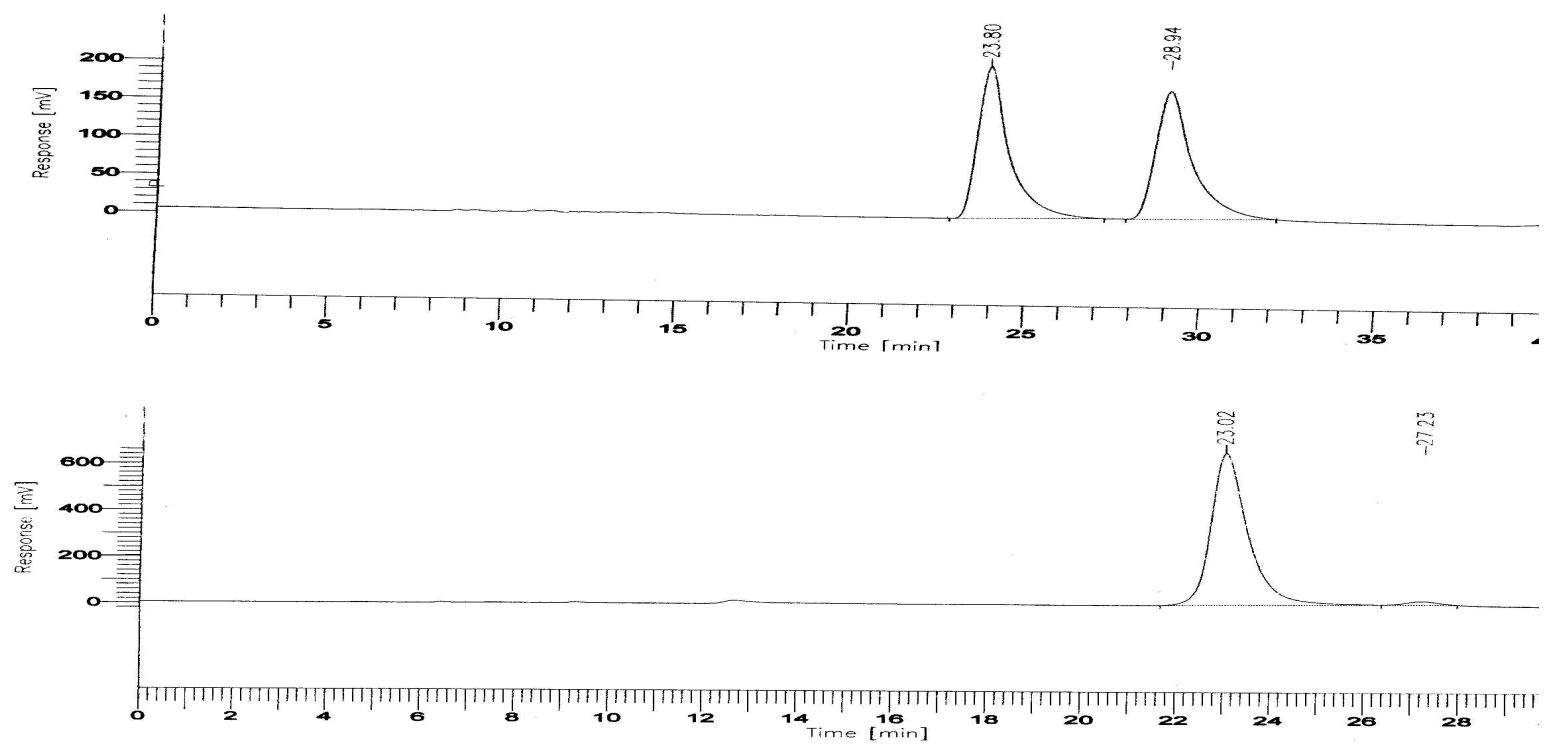

HPLC graph for racemic and enanrioenriched compound (Table 1, Entry 9).

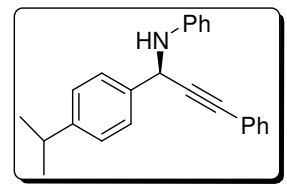

(R)-\{1-(4-Isopropyl-phenyl)-3-phenyl-prop-2-ynyl\}-phenyl amine

(Table 2, Entry 1). 95\% ee; isolated in $89 \%$ yield as colorless gel; $R_{\mathrm{f}}=0.58$ (EtOAc: petroleum ether = 1:19); HPLC conditions: Diacel chiralpak AD-H $(4.6 \mathrm{~cm}$ I.D. x $25 \mathrm{~cm})$, 95:5 hexanes: ${ }^{i}$ - $\mathrm{PrOH}, 0.5 \mathrm{~mL} / \mathrm{min}$ flow rate, $\lambda=254 \mathrm{~nm}, \mathrm{~T}_{\mathrm{R}}$ (major) $=15.52 \mathrm{~min}$ and $\mathrm{T}_{\mathrm{R}}$ $($ minor $)=14.62 \mathrm{~min}$; IR (thin film): 3377, 3019, 2920, 1596, 1492, 1288, 1245, 1057, 982, 846, $753 \mathrm{~cm}-1 ;{ }^{1} \mathrm{H} \mathrm{NMR}\left(\mathrm{CDCl}_{3}, 400 \mathrm{MHz}\right): \delta 7.56(\mathrm{~d}, J=8.04 \mathrm{~Hz}, 2 \mathrm{H}), 7.38-7.40$ $(\mathrm{m}, 2 \mathrm{H}), 7.15-7.25(\mathrm{~m}, 7 \mathrm{H}), 6.75-6.78(\mathrm{~m}, 3 \mathrm{H}), 5.45(\mathrm{~s}, 1 \mathrm{H}), 2.91(\mathrm{~m}, 1 \mathrm{H}), 1.25(\mathrm{~d}, J=$ $6.84 \mathrm{~Hz}, 6 \mathrm{H}) ;{ }^{13} \mathrm{C}\left(\mathrm{CDCl}_{3}, 100 \mathrm{MHz}\right): \delta 148.8,146.6,137.0,131.7,129.1,128.1,127.3$, 126.8, 122.8, 118.4, 113.9, 88.7, 84.8, 50.3, 33.8, 23.9; Anal. cacld. for $\mathrm{C}_{24} \mathrm{H}_{23} \mathrm{~N}$ : C, 
88.57; H, 7.12; N, 4.30. Found: C, 88.75; H, 7.10; N, 4.36; Optical Rotation: $[\alpha]^{25}$ $+82.4\left(\mathrm{c} 0.6, \mathrm{CHCl}_{3}\right)$.
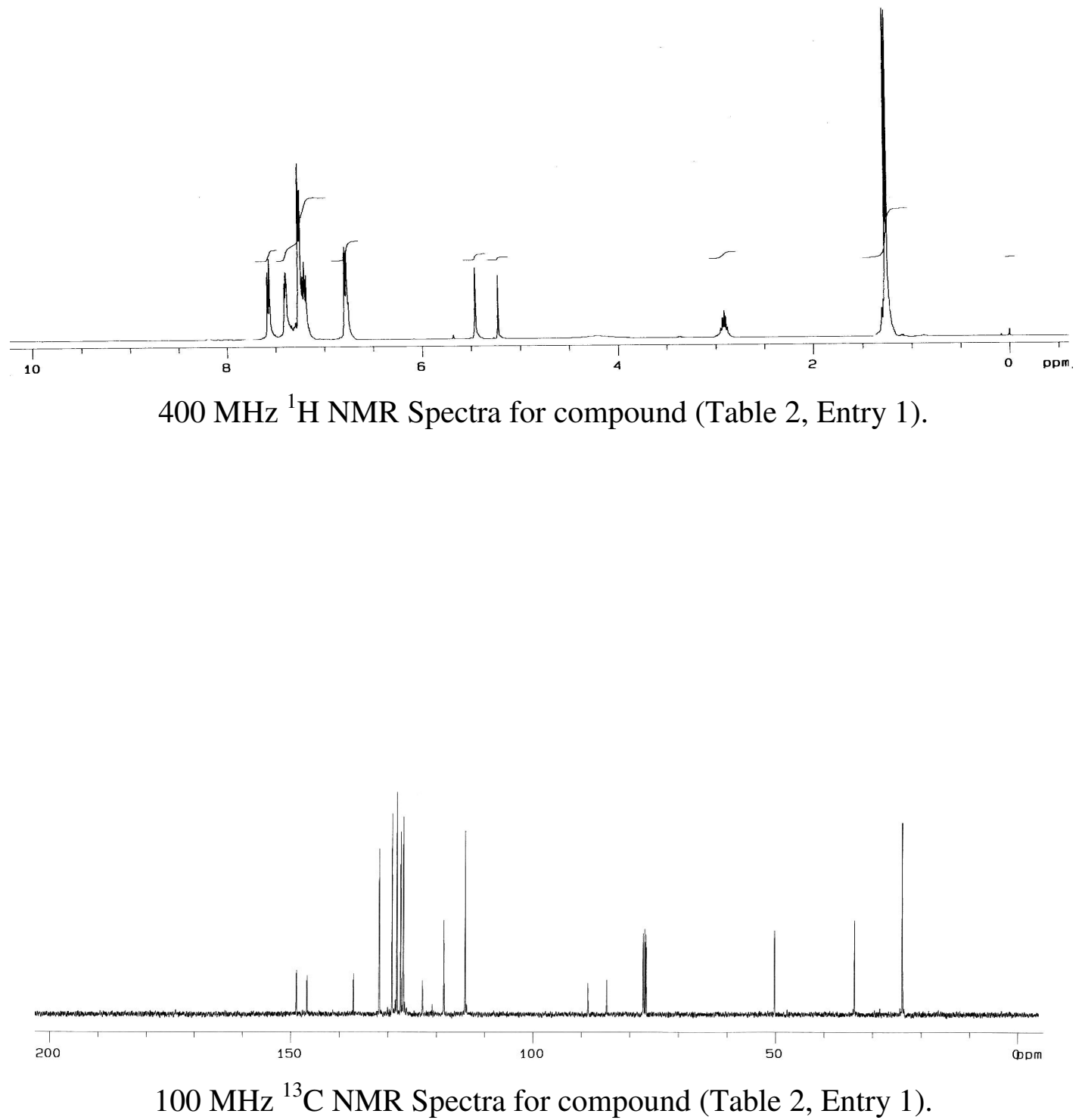

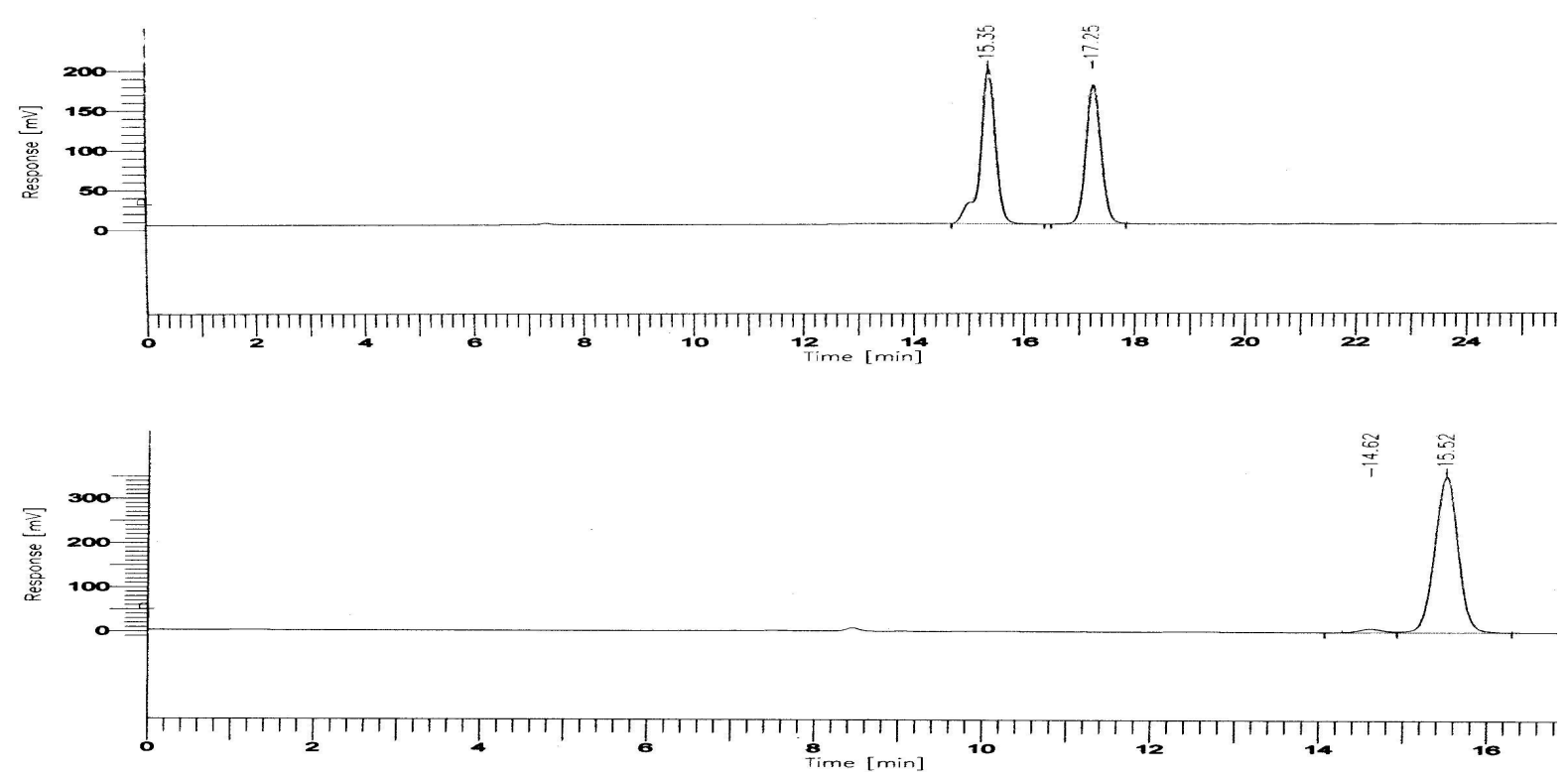

HPLC graph for racemic and enanrioenriched compound (Table 2, Entry 1).

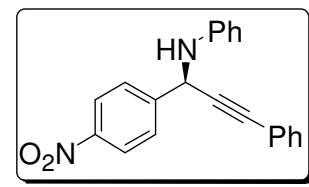

(R)-\{1-(4-Nitro-phenyl)-3-phenyl-prop-2-ynyl\}-phenyl amine

(Table 2, Entry 2). $94.0 \%$ ee; isolated in $82 \%$ yield as yellow solid; $\mathrm{MP}=128-130{ }^{\circ} \mathrm{C}$; $\mathrm{R}_{\mathrm{f}}=0.41$ (EtOAc: petroleum ether $\left.=1: 9\right)$; HPLC conditions: Diacel chiralpak AD-H (4.6 cm I.D. x $25 \mathrm{~cm}$ ), 90:10 hexanes: ${ }^{i}-\mathrm{PrOH}, 0.5 \mathrm{~mL} / \mathrm{min}$ flow rate, $\lambda=254 \mathrm{~nm}, \mathrm{~T}_{\mathrm{R}}$ (major) $=46.20 \mathrm{~min}$ and $\mathrm{T}_{\mathrm{R}}$ (minor) $=40.70 \mathrm{~min}$; Optical Rotation: $[\alpha]_{\mathrm{D}}^{25}+81.1\left(\mathrm{c} 0.7, \mathrm{CHCl}_{3}\right){ }^{2}$
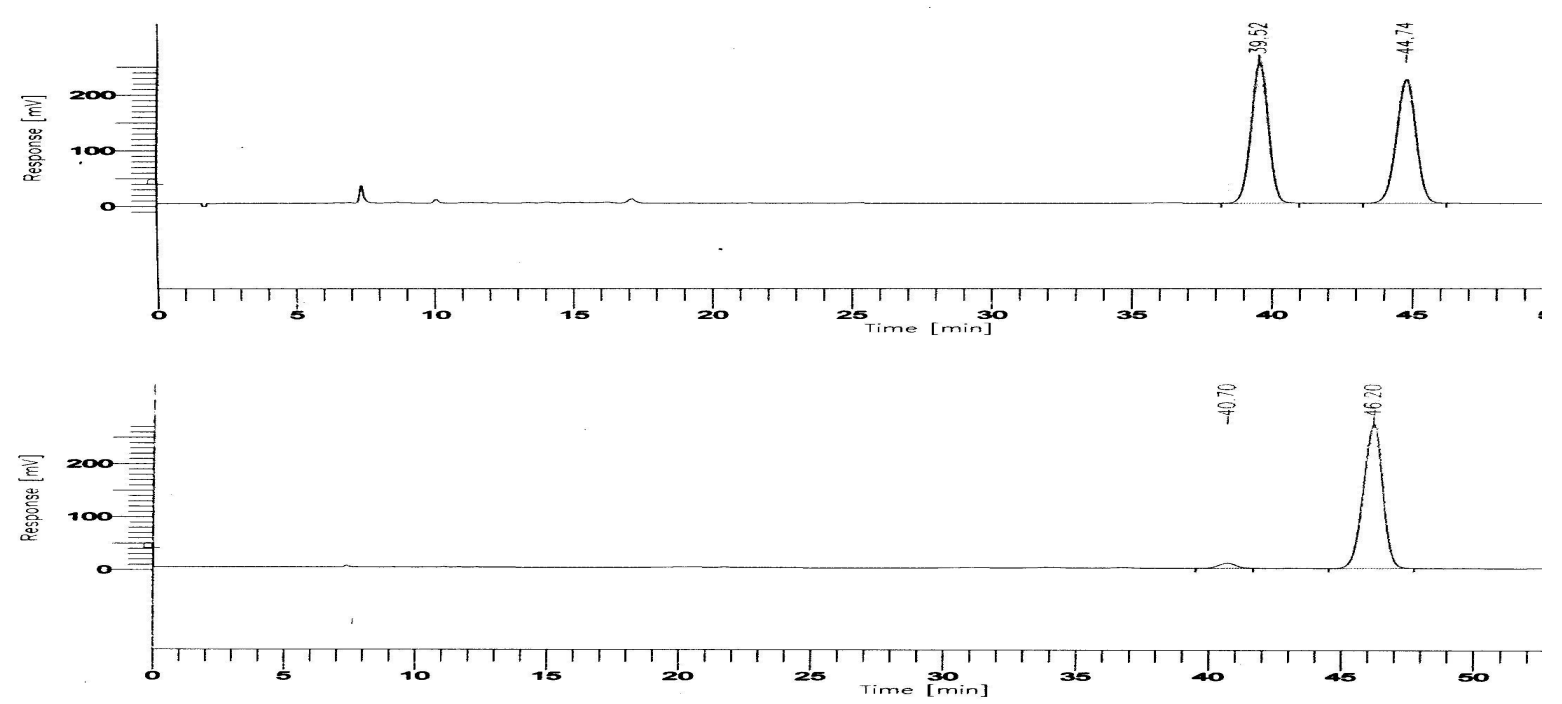

HPLC graph for racemic and enanrioenriched compound (Table 2, Entry 2). 


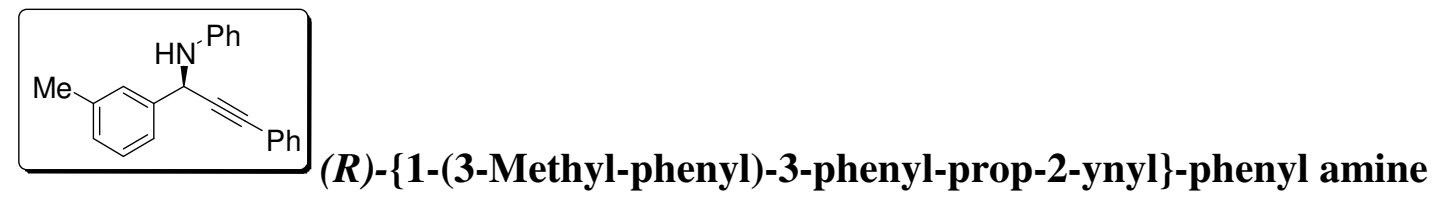

(Table 2, Entry 3). $96 \%$ ee; isolated in $91 \%$ yield as colorless solid; $\mathrm{MP}=113-115{ }^{\circ} \mathrm{C}$; $\mathrm{R}_{\mathrm{f}}=0.59$ (EtOAc: petroleum ether $=1: 19$ ); HPLC conditions: Diacel chiralcel OD-H (4.6 cm I.D. x $25 \mathrm{~cm})$, 99.5:0.5 hexanes: ${ }^{i}-\mathrm{PrOH}, 0.5 \mathrm{~mL} / \mathrm{min}$ flow rate, $\lambda=254 \mathrm{~nm}, \mathrm{~T}_{\mathrm{R}}$ $($ major $)=20.49 \min$ and $\mathrm{T}_{\mathrm{R}}($ minor $)=27.00 \mathrm{~min}$; IR (thin film): 3380, 3049, 2922, 1743, 1594, 1492, 1431, 1297, 1237, 1162, 1096, 786, 750, $690 \mathrm{~cm}-1 ;{ }^{1} \mathrm{H}$ NMR $\left(\mathrm{CDCl}_{3}, 400\right.$ MHz): $\delta$ 7.13-7.45 (m, 11H), 6.76-6.79 (m, 3H), $5.44(\mathrm{~s}, 1 \mathrm{H}), 4.12(\mathrm{bs}, 1 \mathrm{H}, \mathrm{NH}), 2.37$ (s, $3 \mathrm{H}) ;{ }^{13} \mathrm{C}\left(\mathrm{CDCl}_{3}, 100 \mathrm{MHz}\right): \delta 146.6,139.6,138.5,131.7,129.1,128.8,128.6,128.3$, $128.1,127.9,124.3,122.8,118.5,113.9,88.6,84.8,50.6,21.5$; Anal. cacld. for $\mathrm{C}_{22} \mathrm{H}_{19} \mathrm{~N}$ : C, 88.85; H, 6.44; N, 4.71. Found: C, 89.11; H, 6.52; N, 4.69. Optical Rotation: $[\alpha]^{25}$ $+76.5\left(\mathrm{c} 1.1, \mathrm{CHCl}_{3}\right)$.

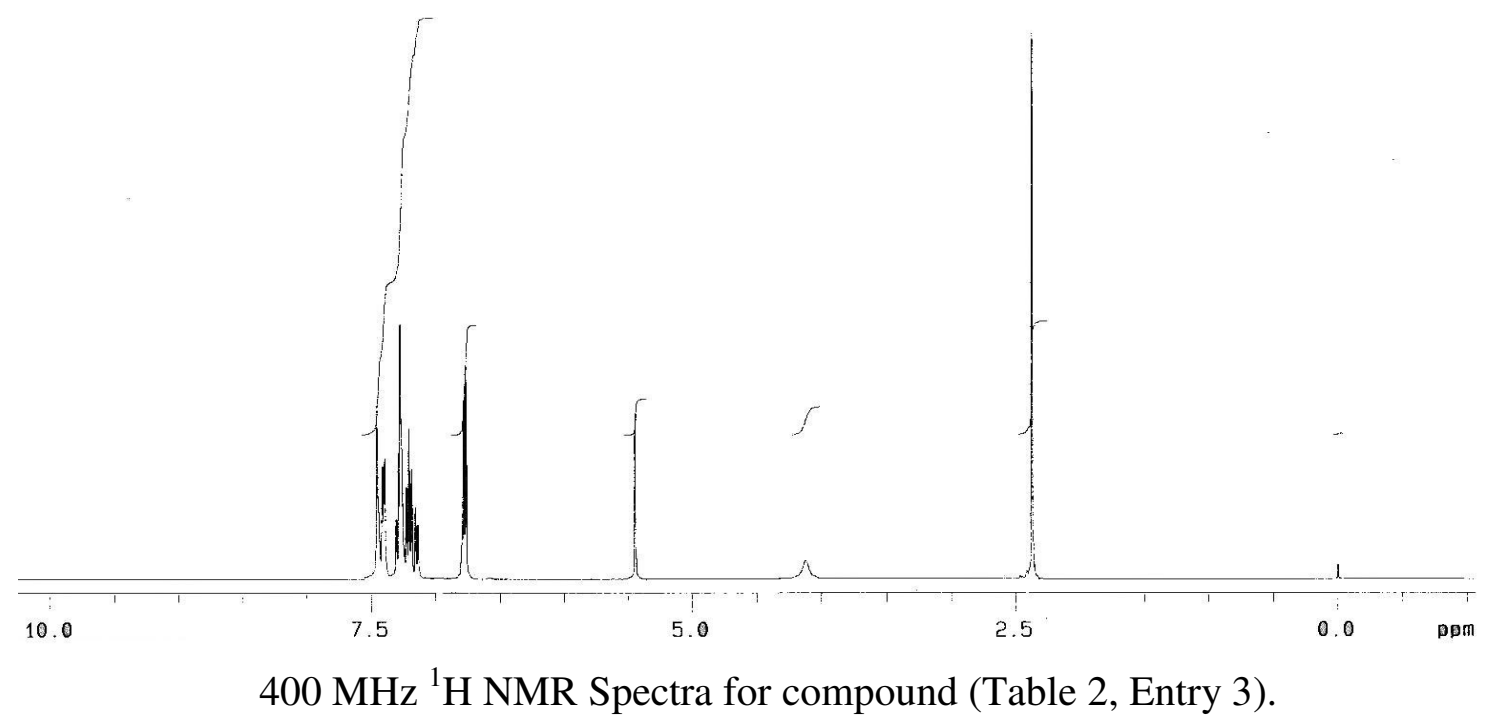




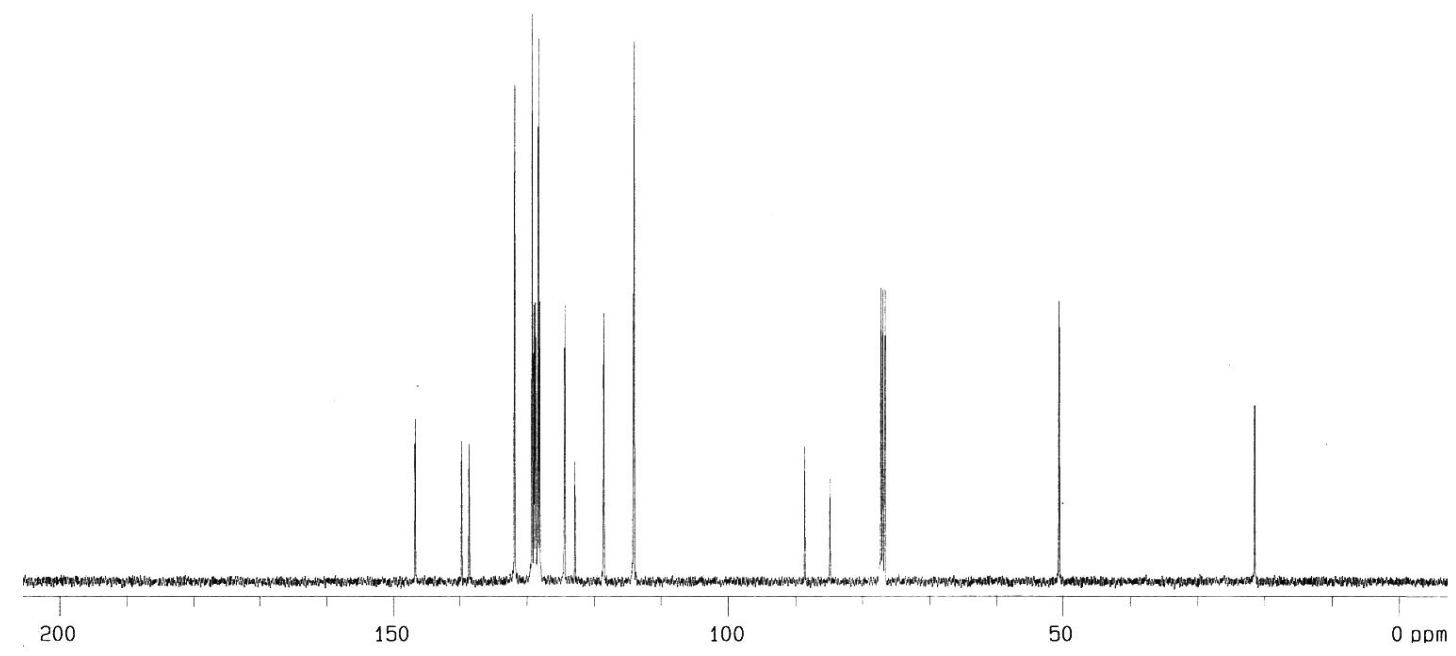

$100 \mathrm{MHz}{ }^{13} \mathrm{C}$ NMR Spectra for compound (Table 2, Entry 3)

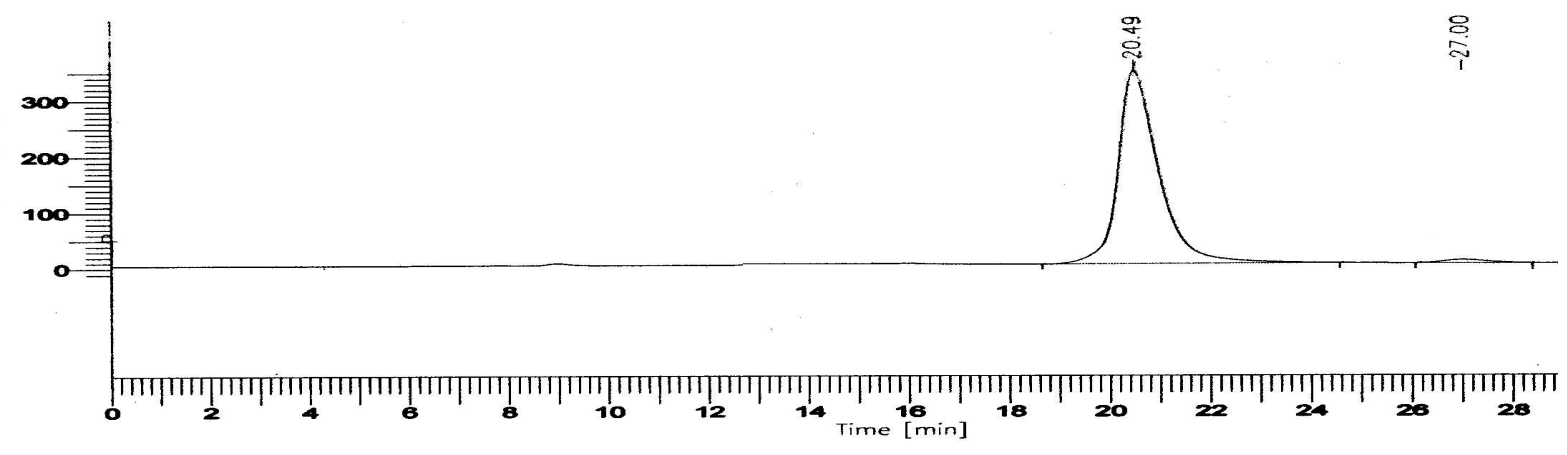

HPLC graph for enanrioenriched compound (Table 2, Entry 3).

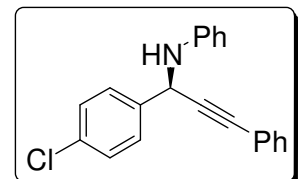

(R)-\{1-(4-Chloro-phenyl)-3-phenyl-prop-2-ynyl\}-phenyl amine

(Table 2, Entry 4). $96 \%$ ee; isolated in $94 \%$ yield as colorless solid; $\mathrm{MP}=78-80{ }^{\circ} \mathrm{C} ; \mathrm{R}_{\mathrm{f}}$ $=0.57$ (EtOAc: petroleum ether = 1:19); HPLC conditions: Diacel chiralcel OD-H (4.6 cm I.D. x $25 \mathrm{~cm}$ ), 99:1 hexanes: ${ }^{i}-\mathrm{PrOH}, 1.0 \mathrm{~mL} / \mathrm{min}$ flow rate, $\lambda=254 \mathrm{~nm}, \mathrm{~T}_{\mathrm{R}}$ (major) $=$ 15.19 min and $\mathrm{T}_{\mathrm{R}}($ minor $)=18.78 \mathrm{~min}$; Optical Rotation: $[\alpha]^{25}{ }_{\mathrm{D}}+80.5\left(\mathrm{c} 0.8, \mathrm{CHCl}_{3}\right){ }^{1,2}$ 


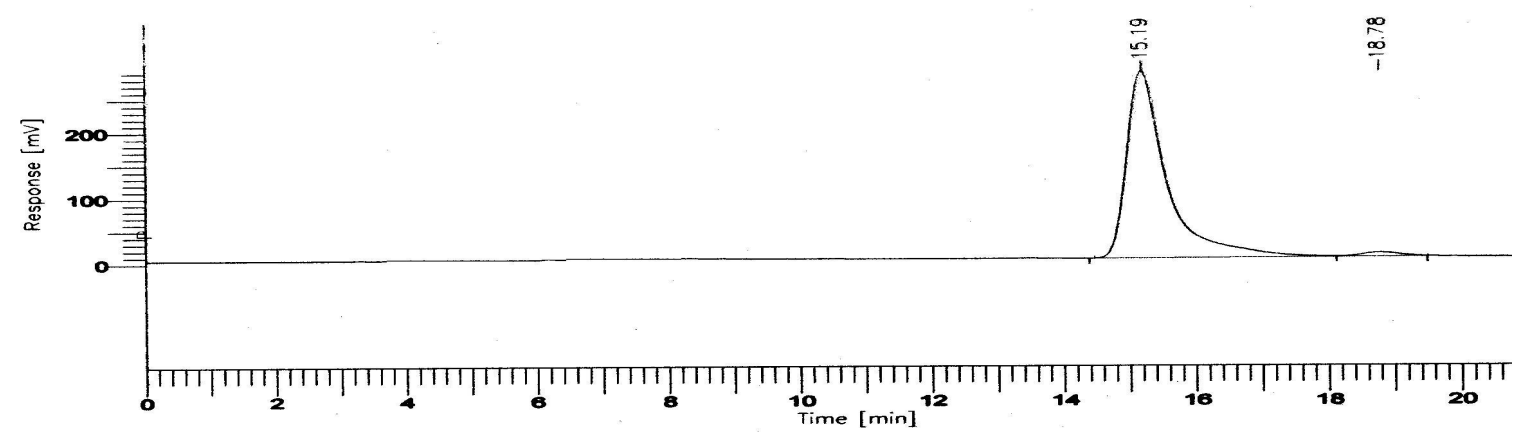

HPLC graph for enanrioenriched compound (Table 2, Entry 4)

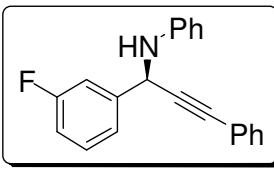

(R)-\{1-(3-Fluoro-phenyl)-3-phenyl-prop-2-ynyl\}-phenyl amine

(Table 2, Entry 5). 93\% ee; isolated in $85 \%$ yield as light yellow solid; $\mathrm{MP}=69-71{ }^{\circ} \mathrm{C}$; $\mathrm{R}_{\mathrm{f}}=0.60$ (EtOAc: petroleum ether $=1: 19$ ); HPLC conditions: Diacel chiralcel OD-H (4.6 cm I.D. $x 25 \mathrm{~cm}$ ), 95:5 hexanes: ${ }^{i}-\mathrm{PrOH}, 0.5 \mathrm{~mL} / \mathrm{min}$ flow rate, $\lambda=254 \mathrm{~nm}, \mathrm{~T}_{\mathrm{R}}$ (major) $=$ $16.99 \mathrm{~min}$ and $\mathrm{T}_{\mathrm{R}}$ (minor) $=21.39 \mathrm{~min}$; IR (thin film): 3376, 3049, 2923, 2852, 1901, 1594, 1492, 1432, 1306, 1182, 1090, 1016, 965, 877, 814, 751, $689 \mathrm{~cm}-1 ;{ }^{1} \mathrm{H}$ NMR $\left(\mathrm{CDCl}_{3}, 400 \mathrm{MHz}\right): \delta 7.18-7.53(\mathrm{~m}, 10 \mathrm{H}), 7.02(\mathrm{dt}, J=8.08,1.96 \mathrm{~Hz}, 1 \mathrm{H}), 6.79(\mathrm{t}, J=$ $7.32 \mathrm{~Hz}, 1 \mathrm{H}), 6.74(\mathrm{~d}, J=7.80 \mathrm{~Hz}, 2 \mathrm{H}), 5.49(\mathrm{~s}, 1 \mathrm{H}), 4.18$ (bs, $1 \mathrm{H}, \mathrm{NH}),{ }^{13} \mathrm{C}\left(\mathrm{CDCl}_{3}, 100\right.$ MHz): $\delta 164.2,161.8,146.2,142.3$ (d), 131.7, 130.2 (d), 129.0, 128.5, 128.2, 122.8 (d), 122.4, 118.8, 115.0, 114.8, 114.3, 114.1, 114.0, 87.7, 85.3, 50.2; Anal. cacld. for $\mathrm{C}_{21} \mathrm{H}_{16} \mathrm{FN}$ : C, 83.70; H, 5.35; N, 4.65. Found: C, 83.93; H, 5.30; N, 4.74. Optical Rotation: $[\alpha]^{25}+99.7$ (c 1.6, $\mathrm{CHCl}_{3}$ ). 


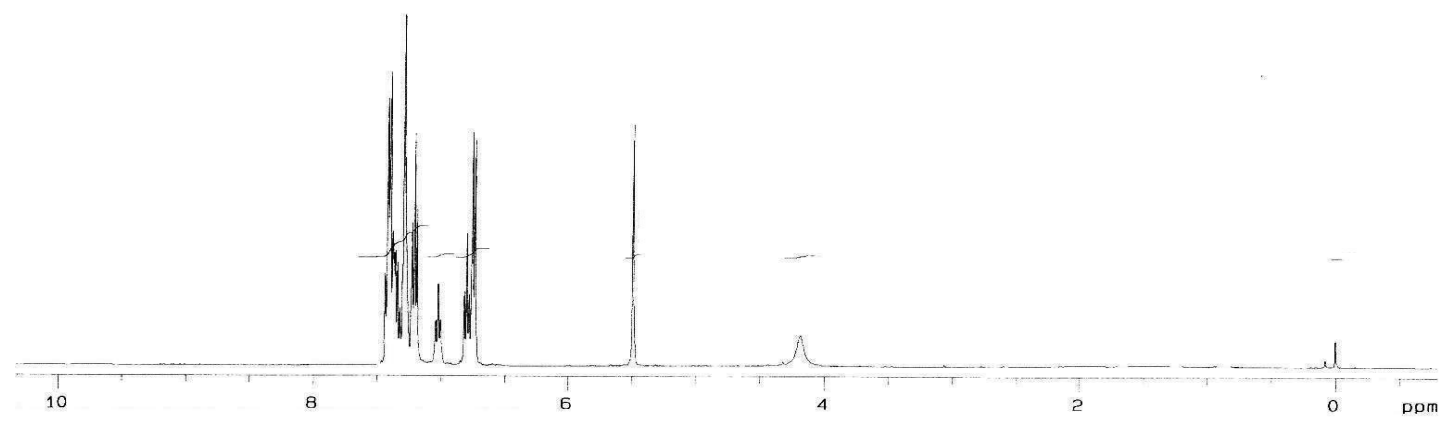

$400 \mathrm{MHz}{ }^{1} \mathrm{H}$ NMR Spectra for compound (Table 2, Entry 5).

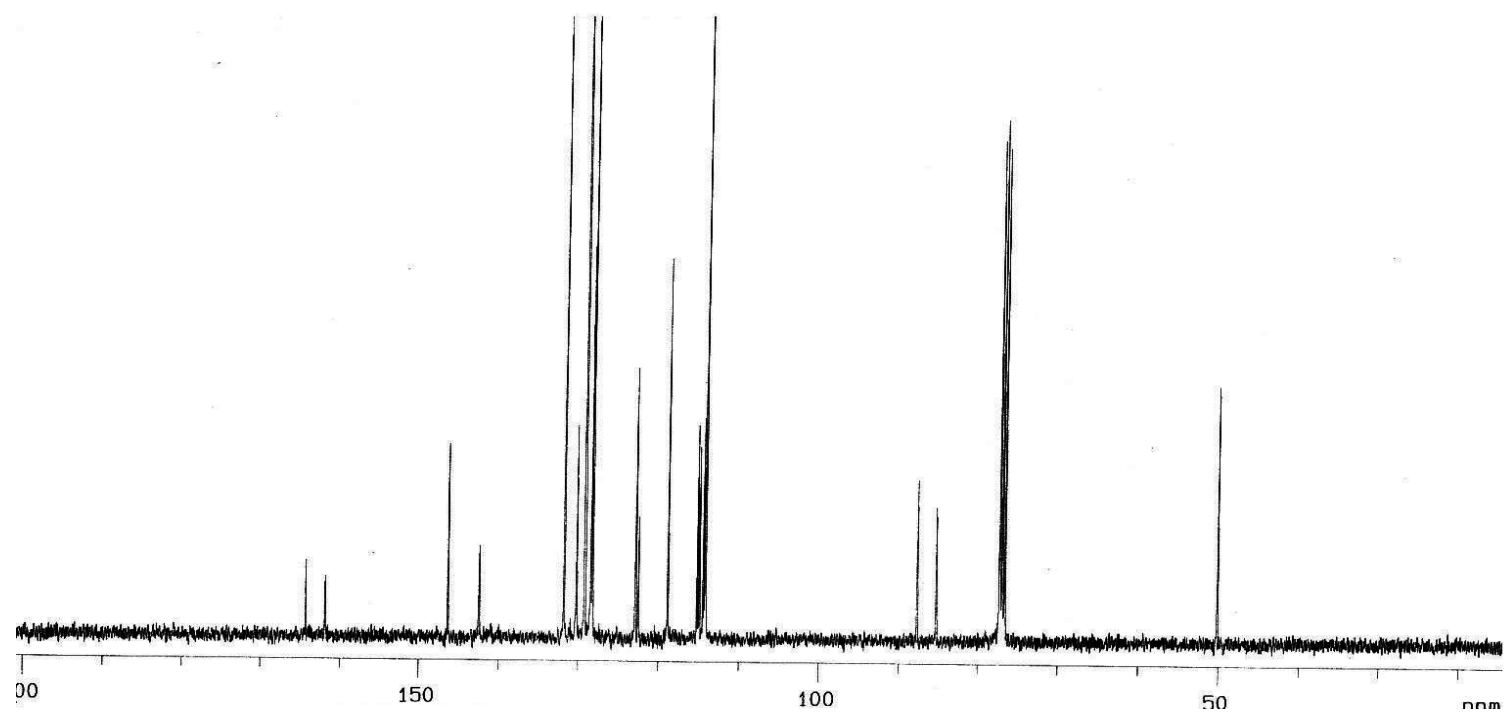

$100 \mathrm{MHz}{ }^{13} \mathrm{C}$ NMR Spectra for compound (Table 2, Entry 5). 


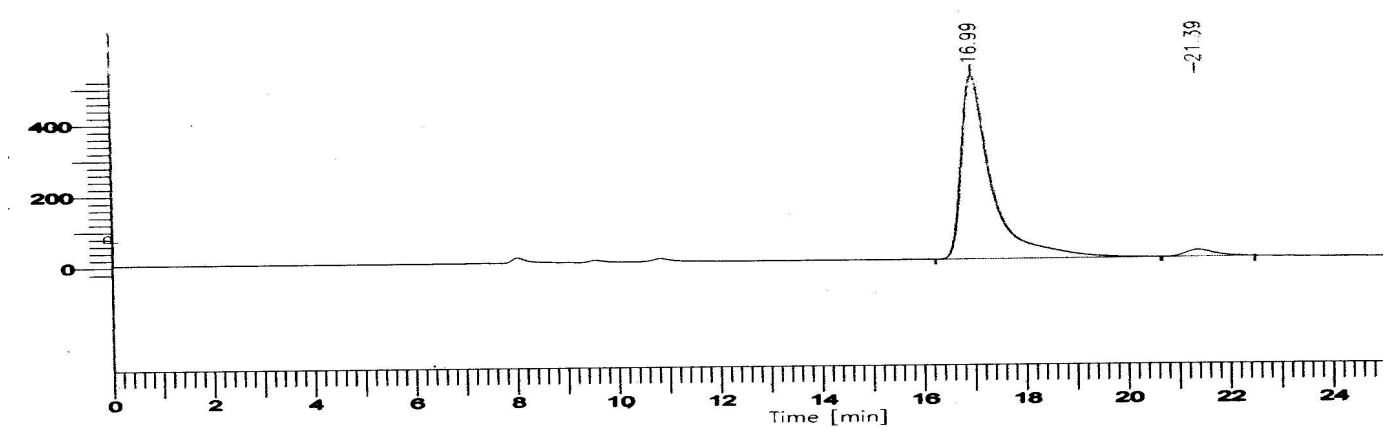

HPLC graph for enanrioenriched compound (Table 2, Entry 5)<smiles>O=C(Nc1ccc(Br)cc1)c1ccccc1</smiles>

\section{(R)-\{1-(3-Bromo-phenyl)-3-phenyl-prop-2-ynyl\}-phenyl amine}

(Table 2, Entry 6). $91 \%$ ee; isolated in $92 \%$ yield as light yellow solid; $\mathrm{MP}=70-72{ }^{\circ} \mathrm{C}$; $\mathrm{R}_{\mathrm{f}}=0.60$ (EtOAc: petroleum ether $\left.=1: 19\right)$; HPLC conditions: Diacel chiralcel OD-H (4.6 cm I.D. x $25 \mathrm{~cm}$ ), 99:1 hexanes: ${ }^{i}-\mathrm{PrOH}, 1.0 \mathrm{~mL} / \mathrm{min}$ flow rate, $\lambda=254 \mathrm{~nm}, \mathrm{~T}_{\mathrm{R}}$ (major) $=$ $14.21 \mathrm{~min}$ and $\mathrm{T}_{\mathrm{R}}($ minor $)=22.74 \mathrm{~min}$; Optical Rotation: $[\alpha]_{\mathrm{D}}^{25}+91.1\left(\mathrm{c} 0.8, \mathrm{CHCl}_{3}\right) .{ }^{2,3}$
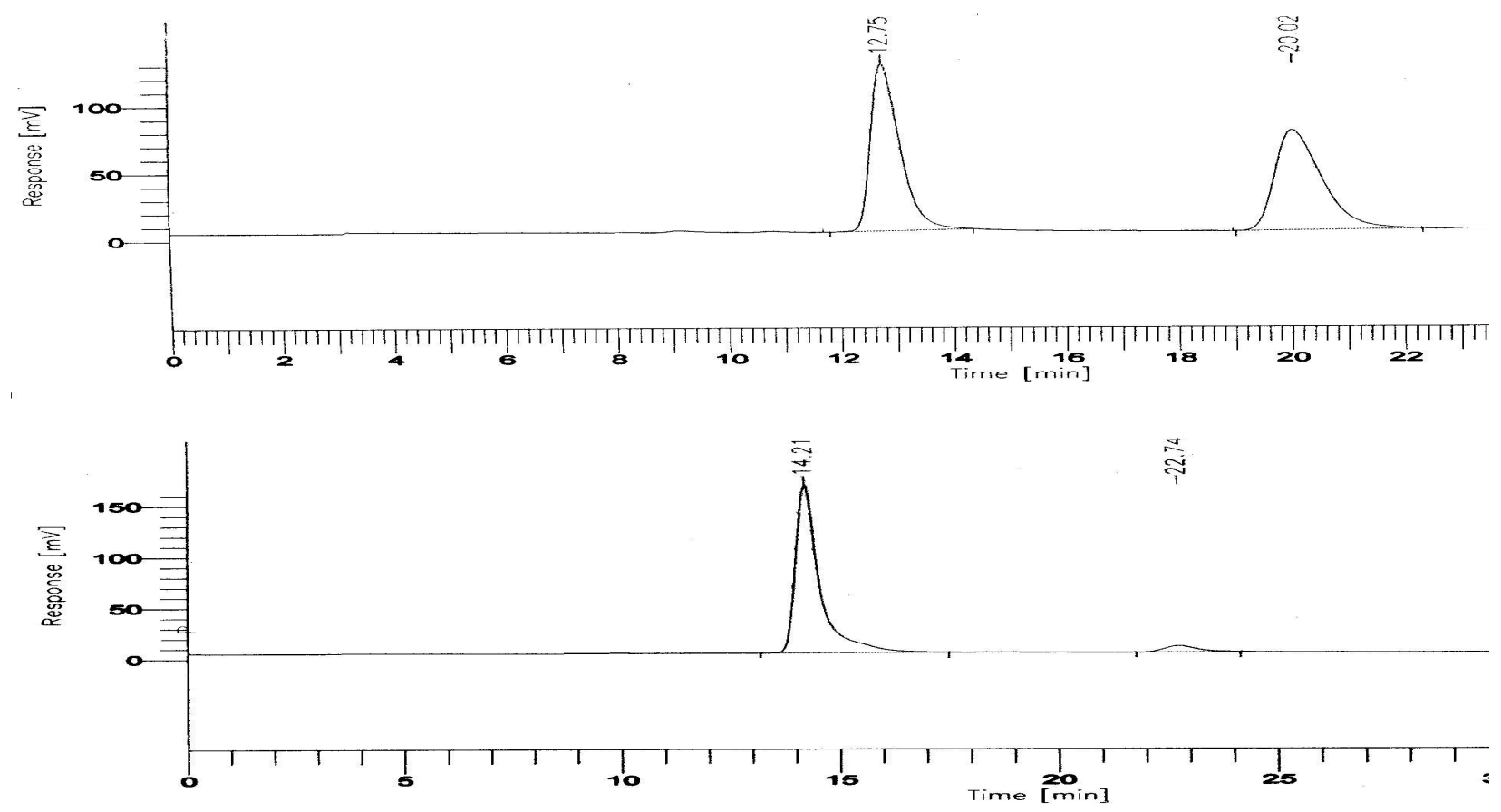

HPLC graph for racemic and enanrioenriched compound (Table 2, Entry 6). 


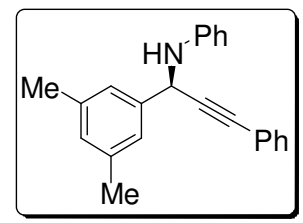

(R)-\{1-(3, 5-Dimethyl-phenyl)-3-phenyl-prop-2-ynyl\}-phenyl amine

(Table 2, Entry 7). $95 \%$ ee; isolated in $98 \%$ yield as colorless solid; $\mathrm{MP}=68-70{ }^{\circ} \mathrm{C}$; $\mathrm{R}_{\mathrm{f}}$ $=0.53$ (EtOAc: petroleum ether =1:19); HPLC conditions: Diacel chiralcel OD-H (4.6 cm I.D. x $25 \mathrm{~cm}$ ), 99:1 hexanes: ${ }^{i}-\mathrm{PrOH}, 0.5 \mathrm{~mL} / \mathrm{min}$ flow rate, $\lambda=254 \mathrm{~nm}, \mathrm{~T}_{\mathrm{R}}$ (major) $=$ $14.46 \mathrm{~min}$ and $\mathrm{T}_{\mathrm{R}}$ (minor) $=19.07 \mathrm{~min}$; IR (thin film): 3377, 3019, 2920, 1596, 1492, 1290, 1243, 1161, 1056, 981, 915, 846, 753, 686, 603, $496 \mathrm{~cm}-1 ;{ }^{1} \mathrm{H}$ NMR $\left(\mathrm{CDCl}_{3}, 400\right.$ $\mathrm{MHz}): \delta 7.38-7.40(\mathrm{~m}, 2 \mathrm{H}), 7.17-7.26(\mathrm{~m}, 7 \mathrm{H}), 6.96(\mathrm{~s}, 1 \mathrm{H}), 6.75-6.78(\mathrm{~m}, 3 \mathrm{H}), 5.39(\mathrm{~s}$, $1 \mathrm{H}), 4.10$ (bs, $1 \mathrm{H}, \mathrm{NH}), 2.33(\mathrm{~s}, 6 \mathrm{H}) ;{ }^{13} \mathrm{C}\left(\mathrm{CDCl}_{3}, 100 \mathrm{MHz}\right): \delta 146.6,139.4,138.3$, 131.7, 129.7, 129.1, 128.1, 125.0, 122.8, 118.4, 113.9, 88.7, 84.7, 50.6, 21.3; Anal. cacld. for $\mathrm{C}_{23} \mathrm{H}_{21} \mathrm{~N}$ : C, 88.71; H, 6.80; N, 4.50. Found: C, 88.96; H, 6.90; N, 4.45. Optical Rotation: $[\alpha]^{25}+140.6\left(\mathrm{c} 2.0, \mathrm{CHCl}_{3}\right)$.

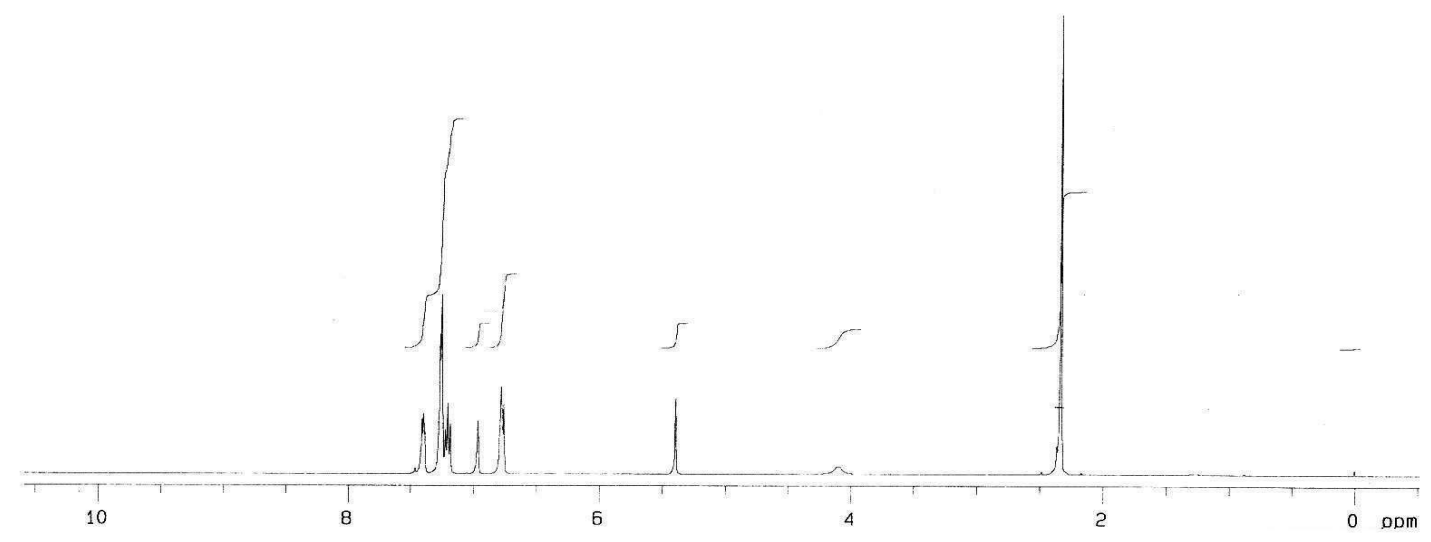

$400 \mathrm{MHz}{ }^{1} \mathrm{H}$ NMR Spectra for compound (Table 2, Entry 7). 


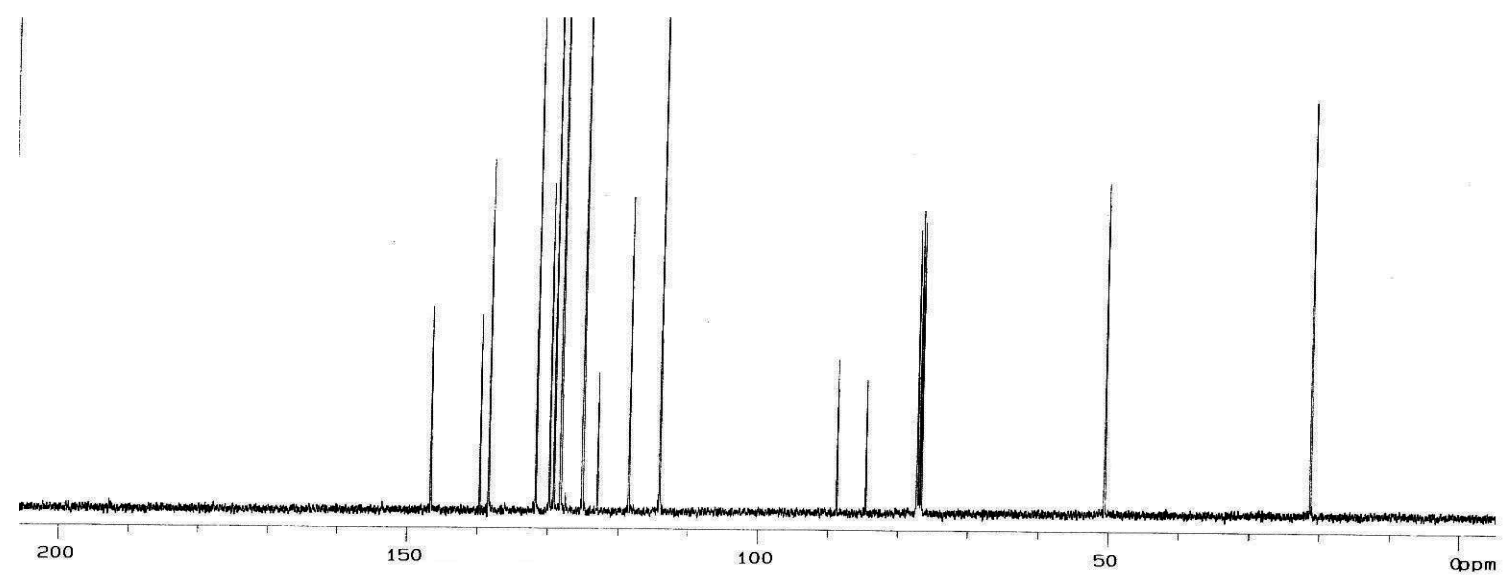

$100 \mathrm{MHz}{ }^{13} \mathrm{C}$ NMR Spectra for compound (Table 2, Entry 7).
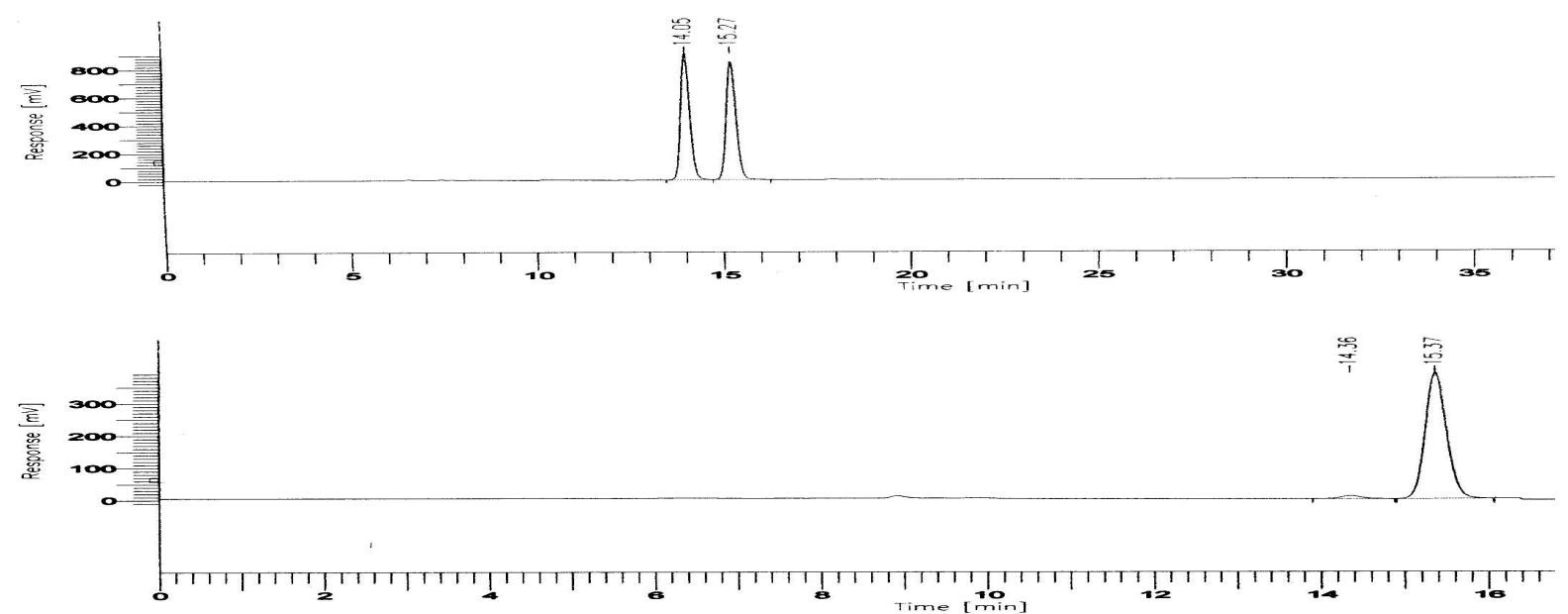

HPLC graph for racemic and enanrioenriched compound (Table 2, Entry 7).<smiles>Fc1ccc(C(C#Cc2ccccc2)Nc2ccccc2)cc1Cl</smiles>

(R)-\{1-(3-Chloro-4-fluoro-phenyl)-3-phenyl-prop-2-ynyl\}-phenyl

amine (Table 2, Entry 8). 91\% ee; isolated in 97\% yield as colorless solid; MP = 77-79 ${ }^{\circ} \mathrm{C} ; \mathrm{R}_{\mathrm{f}}=0.49$ (EtOAc: petroleum ether $=1: 19$ ); HPLC conditions: Diacel chiralcel OD-H (4.6 cm I.D. x $25 \mathrm{~cm}$ ), 95:5 hexanes: ${ }^{i}-\mathrm{PrOH}, 0.5 \mathrm{~mL} / \mathrm{min}$ flow rate, $\lambda=254 \mathrm{~nm}, \mathrm{~T}_{\mathrm{R}}$ $($ major $)=26.81 \mathrm{~min}$ and $\mathrm{T}_{\mathrm{R}}($ minor $)=44.97 \mathrm{~min}$; IR $($ thin film $): 3375,3051,2929,1900$, 
$1594,1494,1434,1284,1235,1161,1089,975,880,815,752,670 \mathrm{~cm}-1 ;{ }^{1} \mathrm{H}$ NMR $\left(\mathrm{CDCl}_{3}, 400 \mathrm{MHz}\right): \delta 7.70(\mathrm{dd}, J=6.96,1.92 \mathrm{~Hz}, 1 \mathrm{H}), 7.48-7.51(\mathrm{~m}, 1 \mathrm{H}), 7.38-7.41(\mathrm{~m}$, 2H), 7.25-7.28 (m, 3H), $7.20(\mathrm{t}, J=8.56 \mathrm{~Hz}, 2 \mathrm{H}), 7.13(\mathrm{t}, J=8.56 \mathrm{~Hz}, 1 \mathrm{H}), 6.80(\mathrm{t}, J=$ $7.32 \mathrm{~Hz}, 1 \mathrm{H}), 6.71(\mathrm{~d}, J=7.80 \mathrm{~Hz}, 2 \mathrm{H}), 5.43(\mathrm{~s}, 1 \mathrm{H}), 4.15$ (bs, $1 \mathrm{H}, \mathrm{NH}) ;{ }^{13} \mathrm{C}\left(\mathrm{CDCl}_{3}, 100\right.$ MHz): $\delta 146.1,141.8,134.6,131.7,130.0,129.9,129.2,128.5,128.3,127.4,125.4$, 122.4, 118.7, 113.9, 87.6, 85.4, 50.2; Anal. cacld. for $\mathrm{C}_{21} \mathrm{H}_{15} \mathrm{ClFN}$ : C, 75.11; H, 4.50; N, 4.17. Found: $\mathrm{C}, 74.99 ; \mathrm{H}, 4.56$; $\mathrm{N}, 4.19$. Optical Rotation: $[\alpha]_{\mathrm{D}}^{25}+42.0\left(\mathrm{c} 0.6, \mathrm{CHCl}_{3}\right)$.

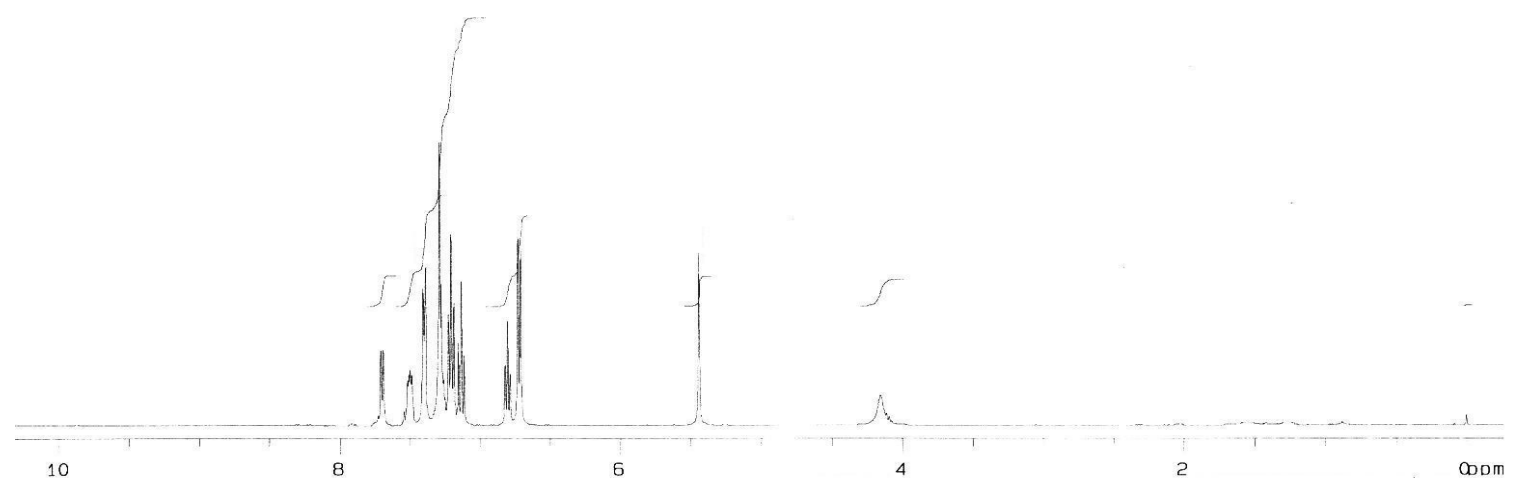
$400 \mathrm{MHz}{ }^{1} \mathrm{H}$ NMR Spectra for compound (Table 2, Entry 8).

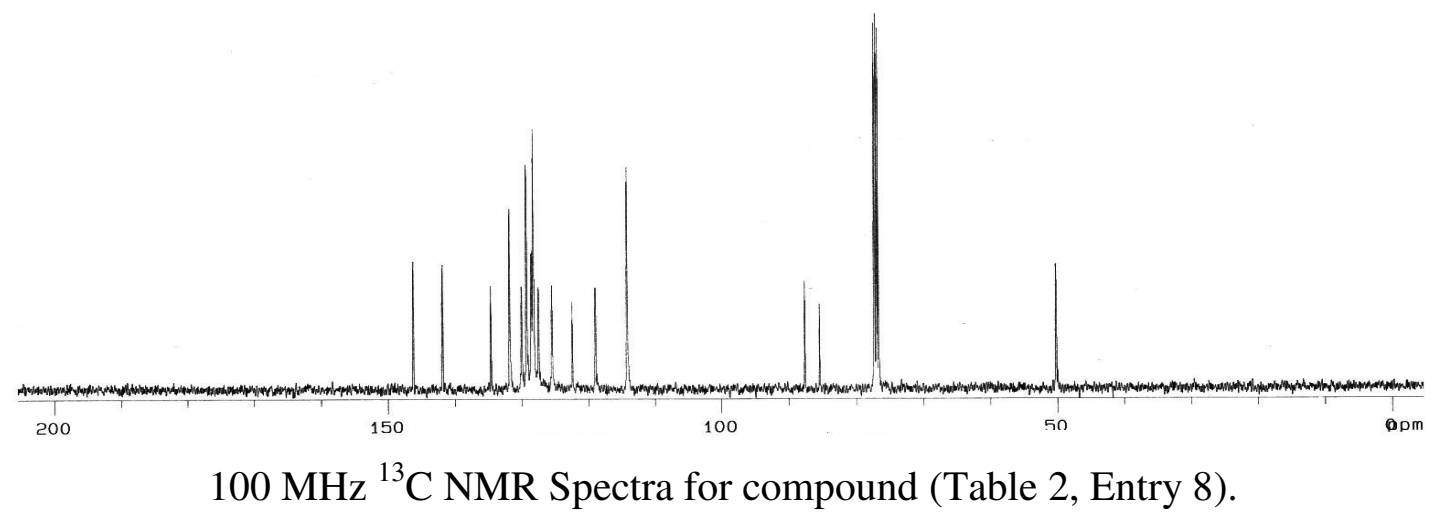




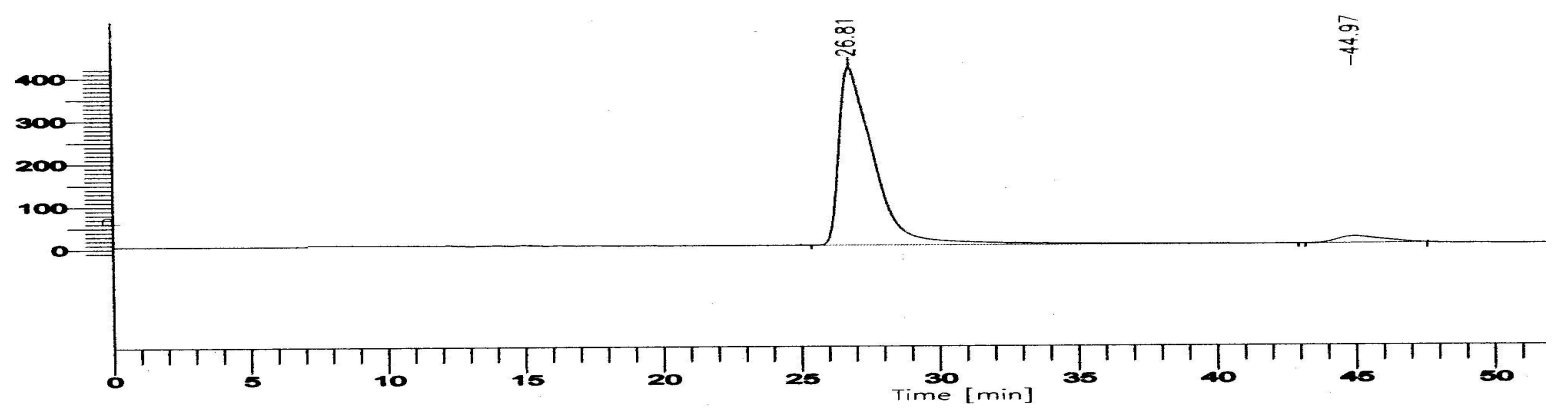

HPLC graph for enanrioenriched compound (Table 2, Entry 8).

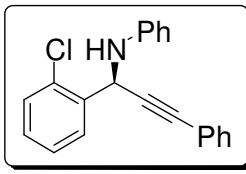

(S)-\{1-(2-Chloro-phenyl)-3-phenyl-prop-2-ynyl\}-phenyl amine (Table

2, Entry 9). 98\% ee; isolated in 93\% yield as light yellow gel; $R_{f}=0.47$ (EtOAc:

petroleum ether = 1:19); HPLC conditions: Diacel chiralpak AD-H $(4.6 \mathrm{~cm}$ I.D. x $25 \mathrm{~cm})$, 95:5 hexanes: ${ }^{i}-\mathrm{PrOH}, 1.0 \mathrm{~mL} / \mathrm{min}$ flow rate, $\lambda=254 \mathrm{~nm}, \mathrm{~T}_{\mathrm{R}}$ (major) $=12.81 \mathrm{~min}$ and $\mathrm{T}_{\mathrm{R}}$ (minor) $=9.66 \mathrm{~min}$; IR (thin film): 3376, 3048, 2926, 1898, 1693, 1594, 1494, 1432, $1284,1236,1161,1089,1018,975,878,813,751,688 \mathrm{~cm}-1 ;{ }^{1} \mathrm{H}$ NMR $\left(\mathrm{CDCl}_{3}, 400\right.$ MHz): $\delta 7.79$ (dd, $J=7.60,2.20 \mathrm{~Hz}, 1 \mathrm{H}), 7.39-7.42(\mathrm{~m}, 3 \mathrm{H}), 7.25-7.31$ (m, 5H), 7.16$7.24(\mathrm{~m}, 2 \mathrm{H}), 6.77(\mathrm{t}, J=7.32 \mathrm{~Hz}, 1 \mathrm{H}), 6.71(\mathrm{~d}, J=7.80 \mathrm{~Hz}, 2 \mathrm{H}), 5.81(\mathrm{~s}, 1 \mathrm{H}), 4.22(\mathrm{bs}$, $1 \mathrm{H}, \mathrm{NH}) ;{ }^{13} \mathrm{C}\left(\mathrm{CDCl}_{3}, 100 \mathrm{MHz}\right): \delta 146.5,139.6,138.5,131.7,129.1,128.8,128.6$, 128.2, 128.1, 127.9, 124.3, 122.7, 118.4, 113.9, 88.6, 84.8, 50.6; Anal. cacld. for $\mathrm{C}_{21} \mathrm{H}_{16} \mathrm{ClN}$ : C, 79.36; H, 5.07; N, 4.41. Found: C, 79.63; H, 5.16; N, 4.43. Optical Rotation: $[\alpha]^{25}{ }_{\mathrm{D}}+98.3\left(\mathrm{c} 0.7, \mathrm{CHCl}_{3}\right){ }^{4}$ 


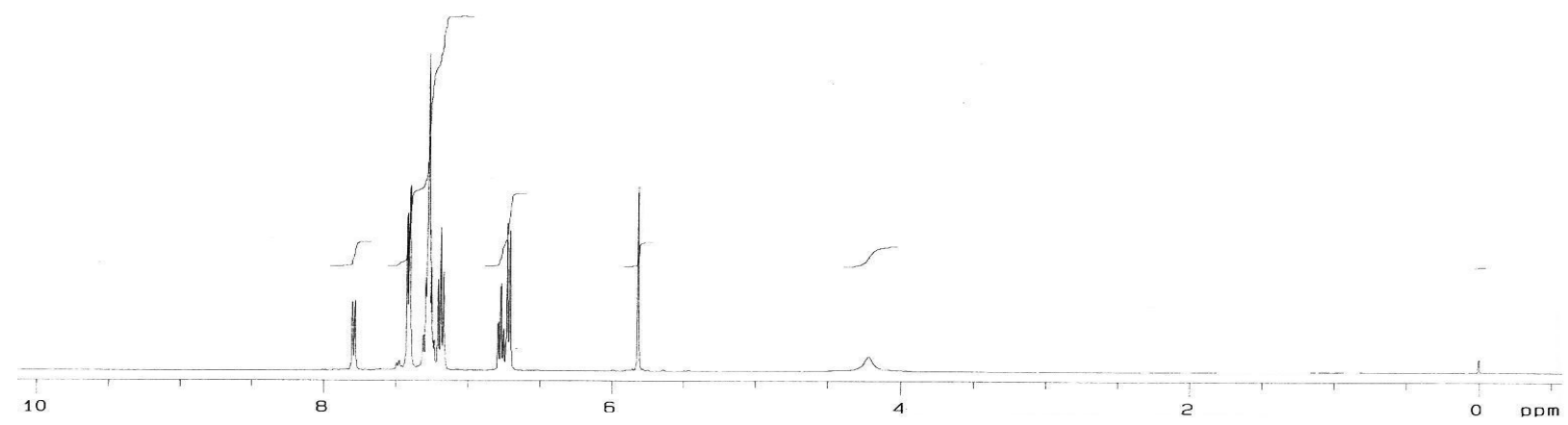

$400 \mathrm{MHz}{ }^{1} \mathrm{H}$ NMR Spectra for compound (Table 2, Entry 9).

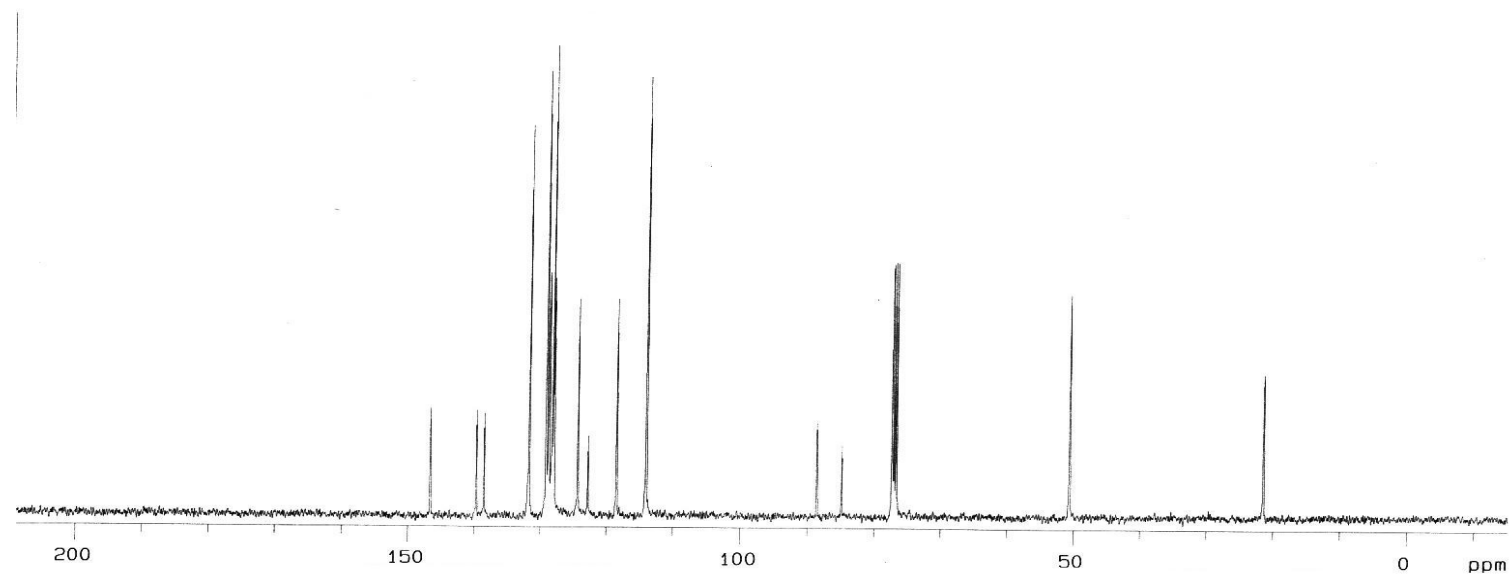

$100 \mathrm{MHz}{ }^{13} \mathrm{C}$ NMR Spectra for compound (Table 2, Entry 9). 

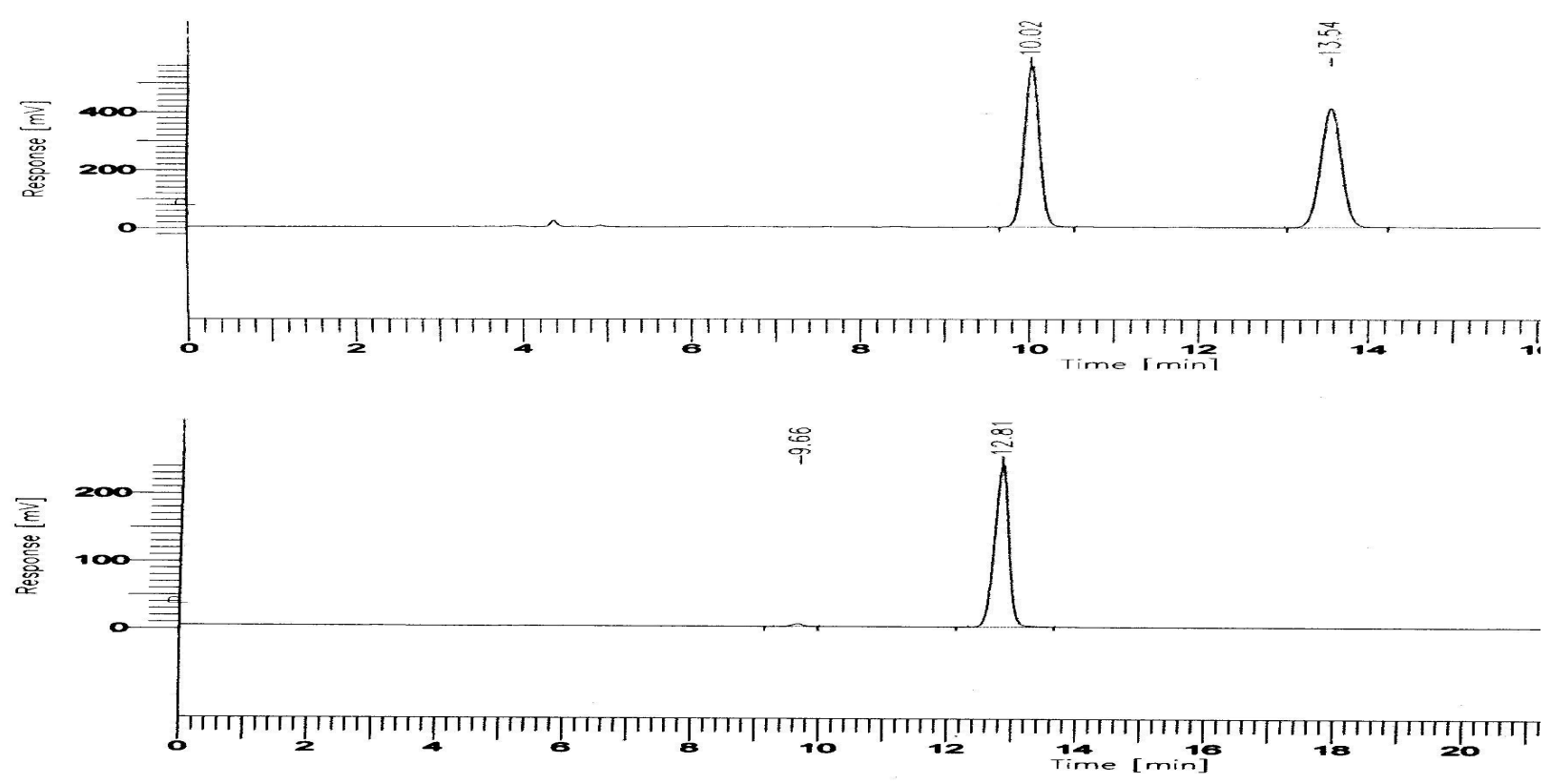

HPLC graph for racemic and enanrioenriched compound (Table 2, Entry 9).

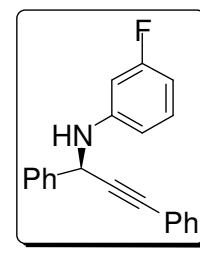

(R)-(1, 3-Diphenyl-prop-2-ynyl)-(3-fluoro-phenyl)-amine (Table 3, Entry

2). $95 \%$ ee; isolated in $93 \%$ yield as colorless solid; $\mathrm{MP}=67-69^{\circ} \mathrm{C} ; \mathrm{R}_{\mathrm{f}}=0.58($ EtOAc: petroleum ether = 1:19); HPLC conditions: Diacel chiralpak AD-H $(4.6 \mathrm{~cm} \mathrm{I.D.} \mathrm{x} 25 \mathrm{~cm})$, 95:5 hexanes: ${ }^{i}-\mathrm{PrOH}, 0.5 \mathrm{~mL} / \mathrm{min}$ flow rate, $\lambda=254 \mathrm{~nm}, \mathrm{~T}_{\mathrm{R}}$ (major) $=21.80 \mathrm{~min}$ and $\mathrm{T}_{\mathrm{R}}$ $($ minor $)=19.71 \mathrm{~min}$; IR (thin film): 3373, 3009, 2913, 2360, 1939, 1710, 1597, 1493, $1435,1290,1241,1163,1053,981,845,751 \mathrm{~cm}-1 ;{ }^{1} \mathrm{H}$ NMR $\left(\mathrm{CDCl}_{3}, 400 \mathrm{MHz}\right): \delta 7.63$ $(\mathrm{d}, J=7.56 \mathrm{~Hz}, 2 \mathrm{H}), 7.24-7.42(\mathrm{~m}, 8 \mathrm{H}), 7.08-7.14(\mathrm{~m}, 1 \mathrm{H}), 6.43-6.51(\mathrm{~m}, 3 \mathrm{H}), 5.45$ (s, $1 \mathrm{H}), 4.26$ (bs, $1 \mathrm{H}, \mathrm{NH}) ;{ }^{13} \mathrm{C}\left(\mathrm{CDCl}_{3}, 100 \mathrm{MHz}\right): \delta 165.0,162.6,148.1(\mathrm{~d}), 139.1,131.7$, 130.2, 129.6, 128.8, 128.2, 127.3, 122.5, 109.8, 105.0 (d), 100.8 (d), 87.8, 85.2, 50.4 (d); Anal. cacld. for $\mathrm{C}_{21} \mathrm{H}_{16} \mathrm{FN}$ : C, 83.70; H, 5.35; N, 4.65. Found: C, 84.02; H, 5.43; N, 4.69. Optical Rotation: $[\alpha]^{25}+129.5\left(\mathrm{c} 2.2, \mathrm{CHCl}_{3}\right)$. 


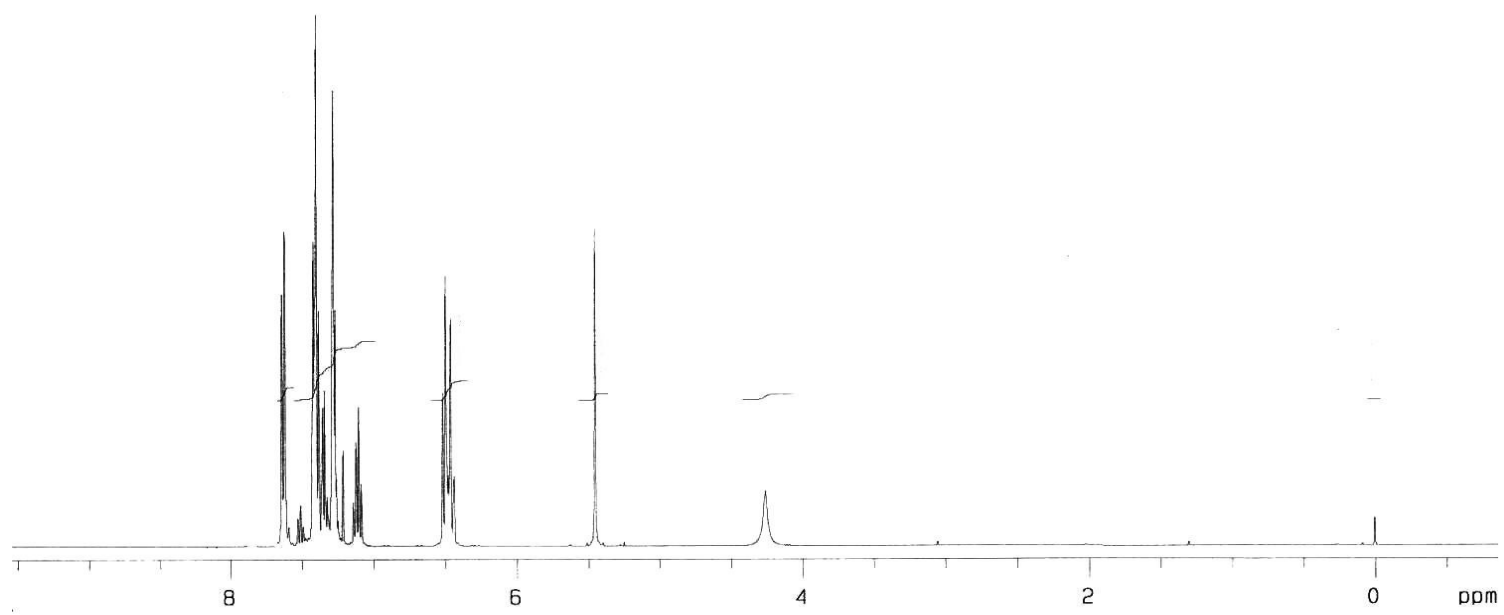

$400 \mathrm{MHz}{ }^{1} \mathrm{H}$ NMR Spectra for compound (Table 3, Entry 2).

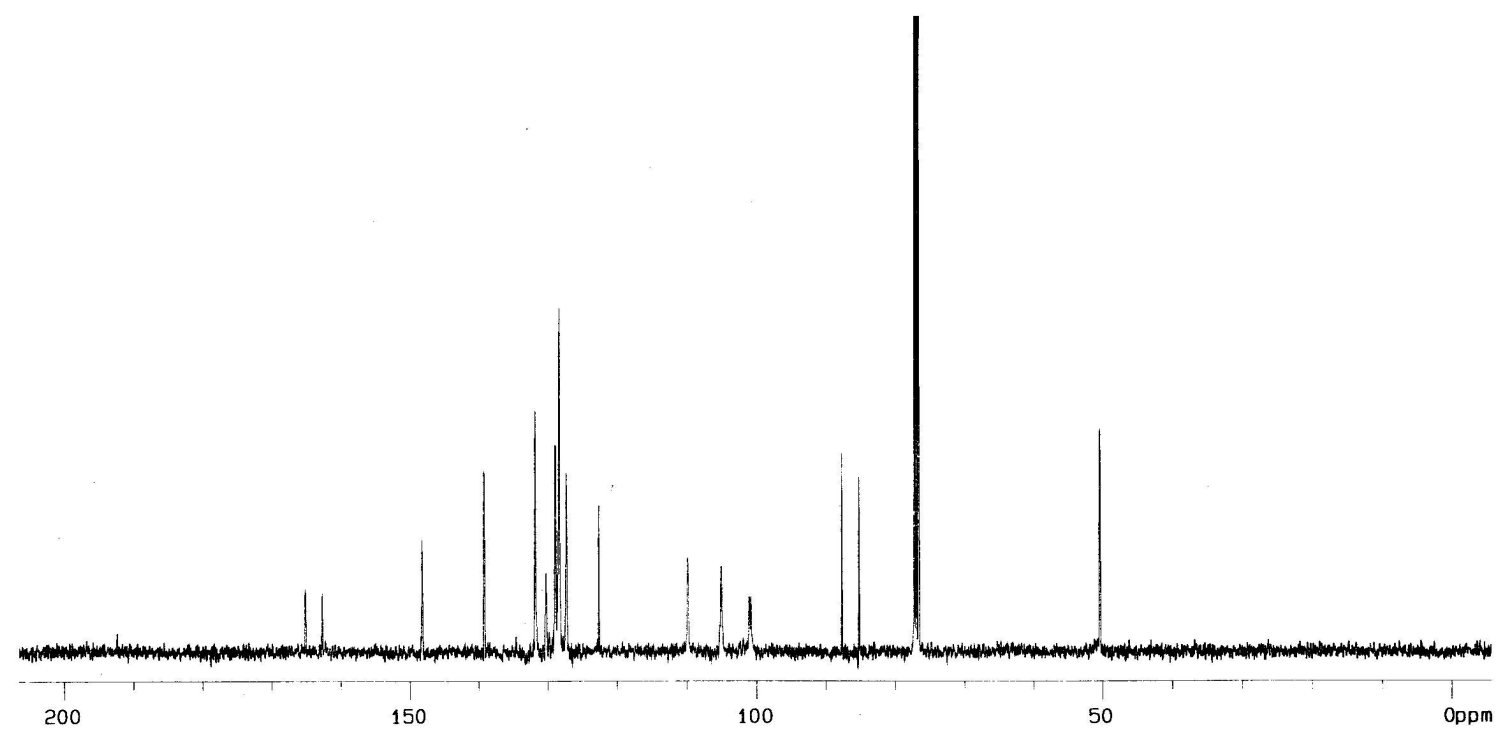

$100 \mathrm{MHz}{ }^{13} \mathrm{C}$ NMR Spectra for compound (Table 3, Entry 2). 

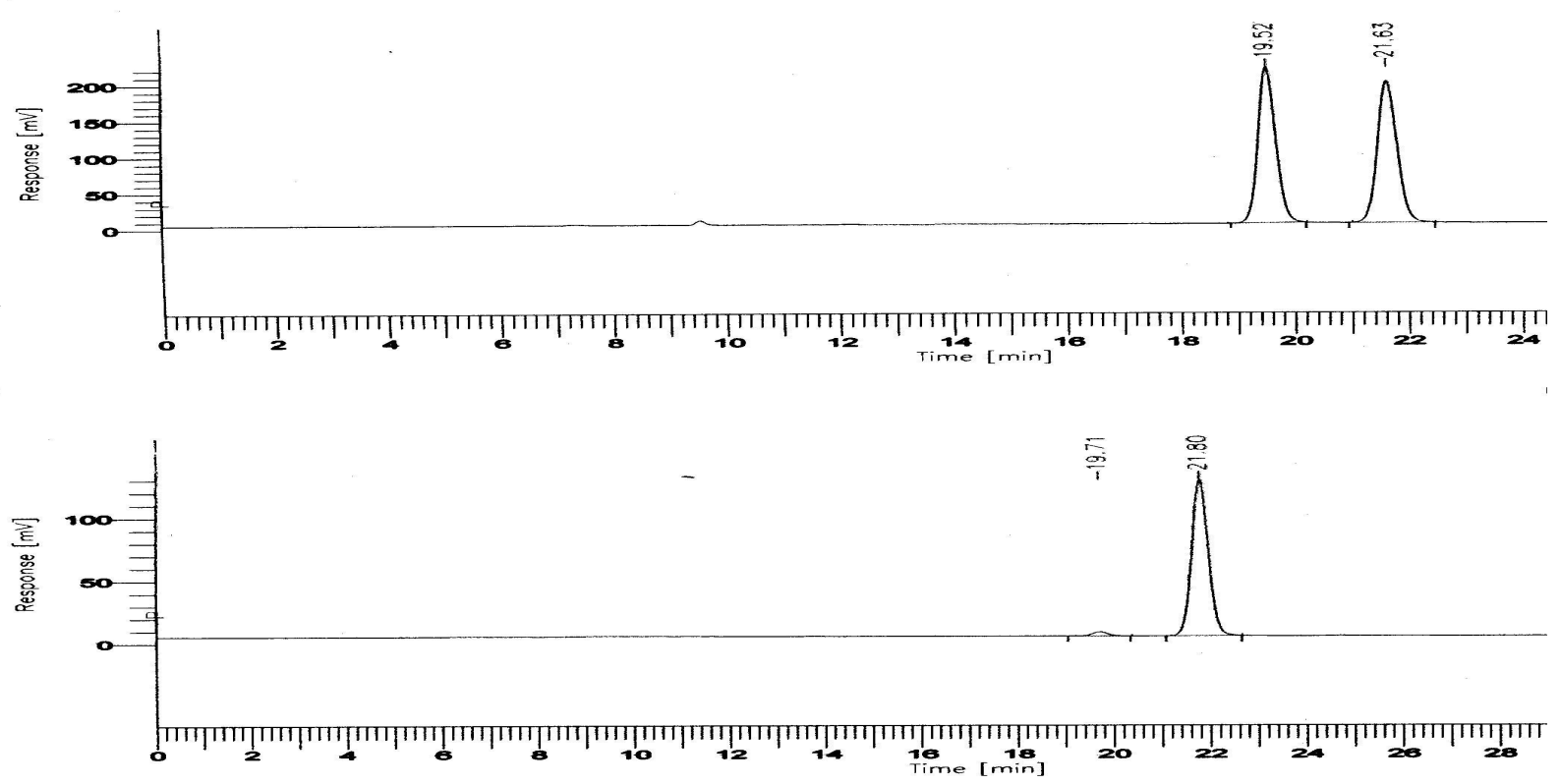

HPLC graph for racemic and enanrioenriched compound (Table 3, Entry 2).

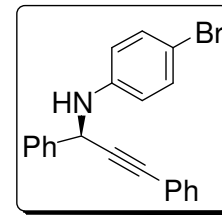

(R)-(1, 3-Diphenyl-prop-2-ynyl)-(4-bromo-phenyl)-amine (Table 3,

Entry 3). $96 \%$ ee; isolated in $86 \%$ yield as light yellow solid; $\mathrm{MP}=75-7{ }^{\circ} \mathrm{C} ; \mathrm{R}_{\mathrm{f}}=0.57$ (EtOAc: petroleum ether =1:19); HPLC conditions: Diacel chiralpak AD-H (4.6 cm I.D. $\mathrm{x} 25 \mathrm{~cm}$ ), 95:5 hexanes: ${ }^{i} \mathrm{PrOH}, 0.5 \mathrm{~mL} / \mathrm{min}$ flow rate, $\lambda=254 \mathrm{~nm}, \mathrm{~T}_{\mathrm{R}}$ (major) $=27.10$ $\min$ and $\mathrm{T}_{\mathrm{R}}($ minor $)=23.10 \mathrm{~min}$; Optical Rotation: $[\alpha]^{25}+124.1\left(\mathrm{c} 2.4, \mathrm{CHCl}_{3}\right) .{ }^{1,2,3}$ 

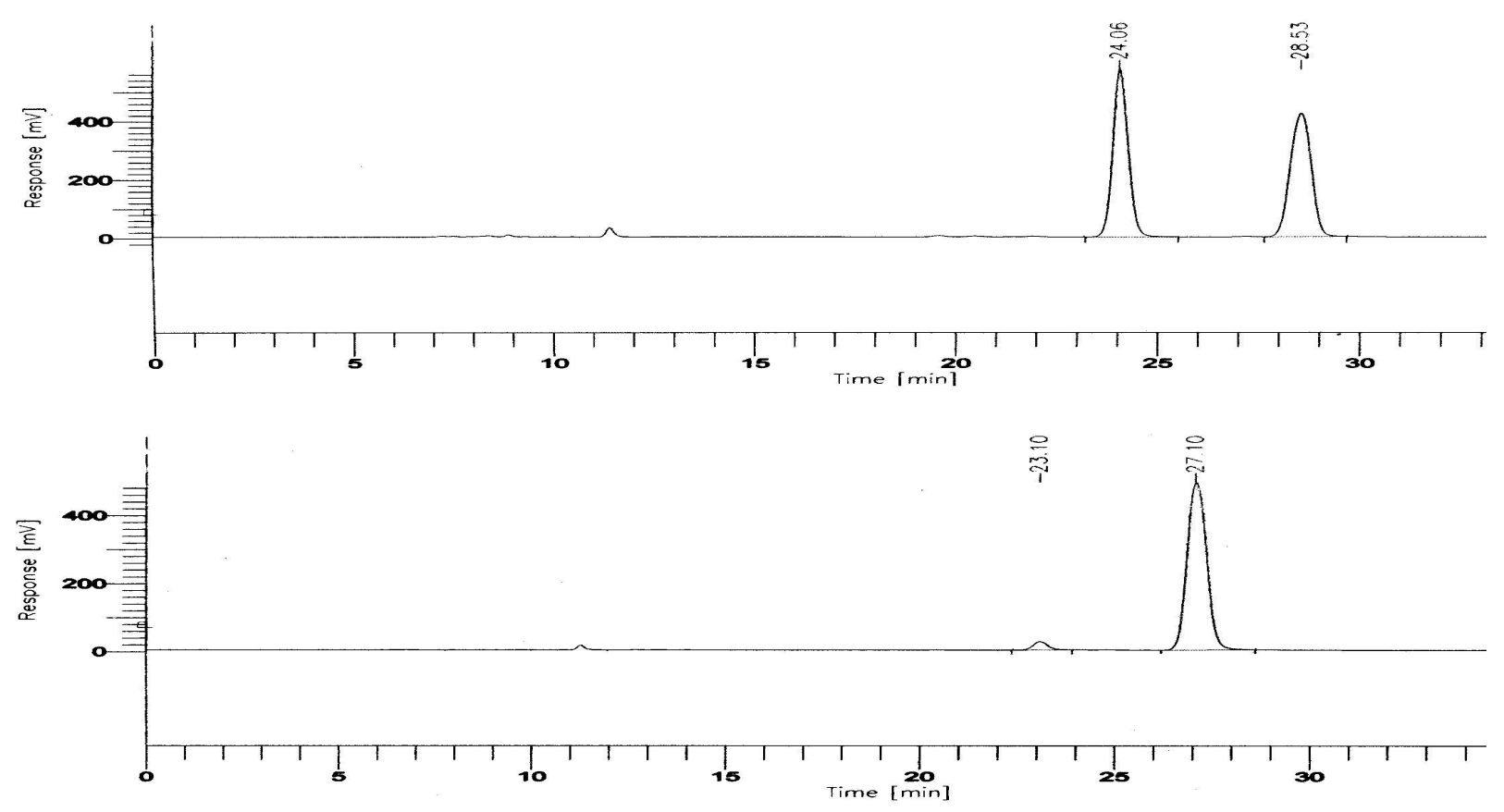

HPLC graph for racemic and enanrioenriched compound (Table 3, Entry 3).

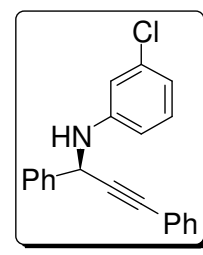

(R)-(1, 3-Diphenyl-prop-2-ynyl)-(3-chloro-phenyl)-amine (Table 3, Entry

4). $95 \%$ ee; isolated in $93 \%$ yield as light yellow gel; $\mathrm{R}_{\mathrm{f}}=0.51$ (EtOAc: petroleum ether = 1:19); HPLC conditions: Diacel chiralpak AD-H (4.6 cm I.D. x $25 \mathrm{~cm}), 95: 5$ hexanes: ${ }^{i}-$ $\mathrm{PrOH}, 0.5 \mathrm{~mL} / \mathrm{min}$ flow rate, $\lambda=254 \mathrm{~nm}, \mathrm{~T}_{\mathrm{R}}$ (major) $=22.00 \mathrm{~min}$ and $\mathrm{T}_{\mathrm{R}}$ (minor) $=$ $20.91 \mathrm{~min}$; IR (thin film): 3375, 3012, 2916, 2362, 1940, 1711, 1596, 1492, 1436, 1289, 1243, 1162, 1055, 983, 915, 847, 753, $687 \mathrm{~cm}-1 ;{ }^{1} \mathrm{H}$ NMR $\left(\mathrm{CDCl}_{3}, 400 \mathrm{MHz}\right): \delta 7.62$ (d, $J=7.32 \mathrm{~Hz}, 2 \mathrm{H}), 7.25-7.42(\mathrm{~m}, 8 \mathrm{H}), 7.10(\mathrm{~m}, 1 \mathrm{H}), 6.73-6.77(\mathrm{~m}, 2 \mathrm{H}), 6.60-6.63(\mathrm{~m}, 1 \mathrm{H})$, $5.45(\mathrm{~s}, 1 \mathrm{H}), 4.22(\mathrm{bs}, 1 \mathrm{H}, \mathrm{NH}) ;{ }^{13} \mathrm{C}\left(\mathrm{CDCl}_{3}, 100 \mathrm{MHz}\right): \delta 147.6,139.1,134.8,131.7$, $130.1,129.7,128.8,128.4,128.2,127.2,122.5,118.4,113.8,112.2,87.7,85.3,50.4$; Anal. cacld. for $\mathrm{C}_{21} \mathrm{H}_{16} \mathrm{ClN}$ : C, 79.36; H, 5.07; N, 4.41. Found: C, 79.61; H, 5.14; N, 4.52. Optical Rotation: $[\alpha]^{25}+77.3\left(\mathrm{c} 0.7, \mathrm{CHCl}_{3}\right)$. 


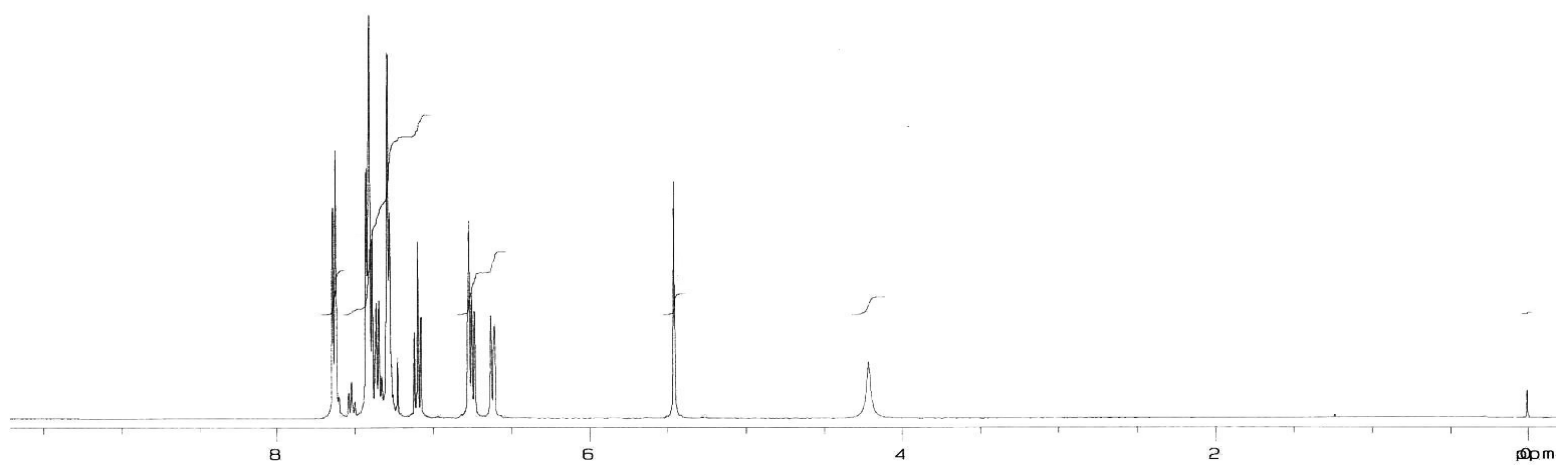

$400 \mathrm{MHz}{ }^{1} \mathrm{H}$ NMR Spectra for compound (Table 3, Entry 4).

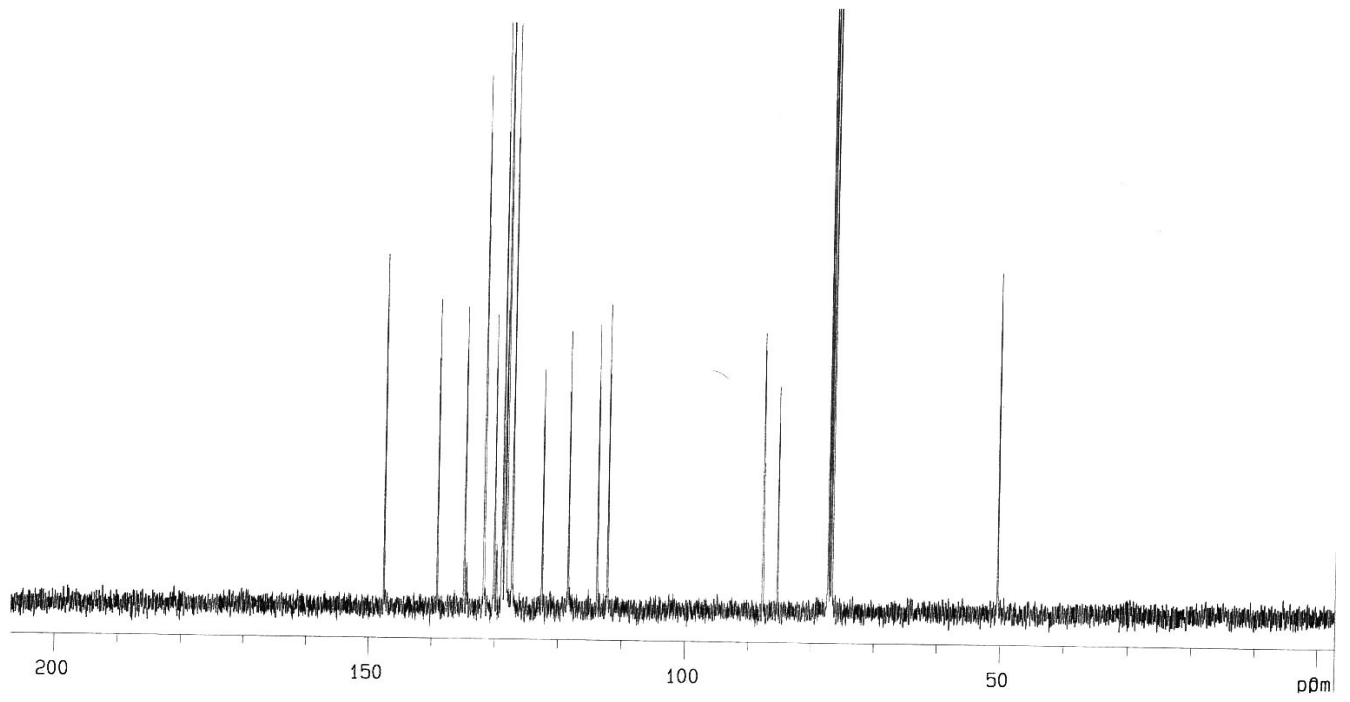

$100 \mathrm{MHz}{ }^{13} \mathrm{C}$ NMR Spectra for compound (Table 3, Entry 4). 

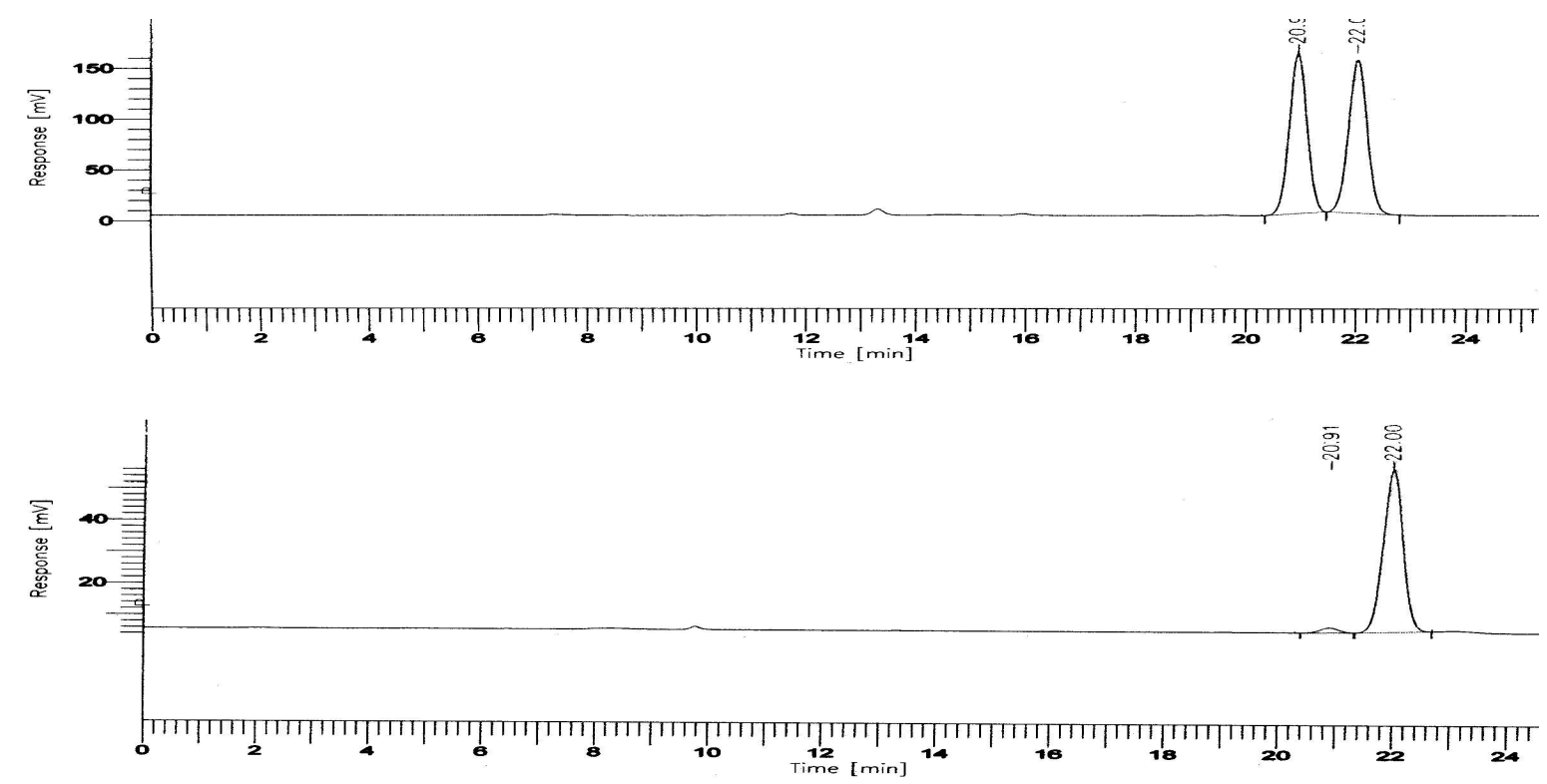

HPLC graph for racemic and enanrioenriched compound (Table 3, Entry 4).

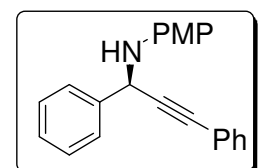

\section{(R)-(1, 3-Diphenyl-prop-2-ynyl)-(4-methoxy-phenyl)-amine (Table 3,}

Entry 6). $90 \%$ ee; isolated in $98 \%$ yield as yellow gel; $R_{\mathrm{f}}=0.49$ (EtOAc: petroleum ether = 1:9); HPLC conditions: Diacel chiralcel OD-H (4.6 cm I.D. x $25 \mathrm{~cm}$ ), 95:5 hexanes: ${ }^{i}-$ $\mathrm{PrOH}, 1.0 \mathrm{~mL} / \mathrm{min}$ flow rate, $\lambda=254 \mathrm{~nm}, \mathrm{~T}_{\mathrm{R}}$ (major) $=33.79 \mathrm{~min}$ and $\mathrm{T}_{\mathrm{R}}$ (minor) $=$ 28.36 min; IR (thin film): 3369, 2927, 2857, 1736, 1601, 1511, 1460, 1240, 1172, 1034, 821, 738, $697 \mathrm{~cm}-1 ;{ }^{1} \mathrm{H} \mathrm{NMR}\left(\mathrm{CDCl}_{3}, 400 \mathrm{MHz}\right): \delta 7.64(\mathrm{~d}, J=7.32 \mathrm{~Hz}, 2 \mathrm{H}), 7.22-7.60$ $(\mathrm{m}, 8 \mathrm{H}), 6.72-6.80(\mathrm{~m}, 4 \mathrm{H}), 5.39(\mathrm{~s}, 1 \mathrm{H}), 3.71(\mathrm{~s}, 3 \mathrm{H}) ;{ }^{13} \mathrm{C}\left(\mathrm{CDCl}_{3}, 100 \mathrm{MHz}\right): \delta 140.6$, 139.8, 131.7, 128.7, 128.2, 128.1, 127.9, 127.3, 122.7, 115.8, 114.6, 88.7, 85.0, 55.6, 51.7; Anal. cacld. for $\mathrm{C}_{22} \mathrm{H}_{19} \mathrm{NO}$ : C, 84.31; H, 6.11; N, 4.47. Found: C, 84.59; H, 6.19; N, 4.49. Optical Rotation: $[\alpha]^{25}+129.5\left(\mathrm{c} 1.0, \mathrm{CHCl}_{3}\right)^{5}$ 

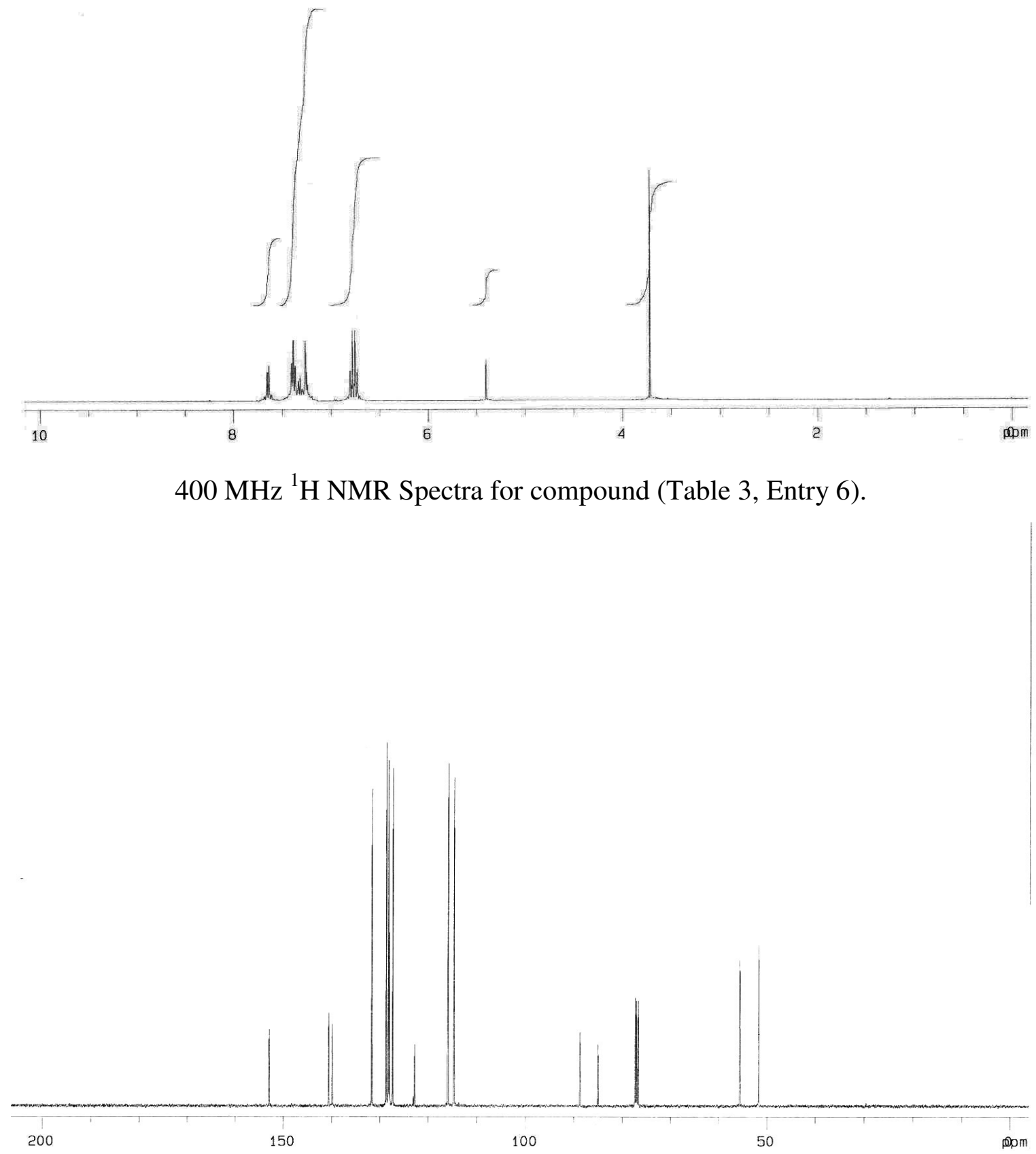

$100 \mathrm{MHz}{ }^{13} \mathrm{C}$ NMR Spectra for compound (Table 3, Entry 6). 

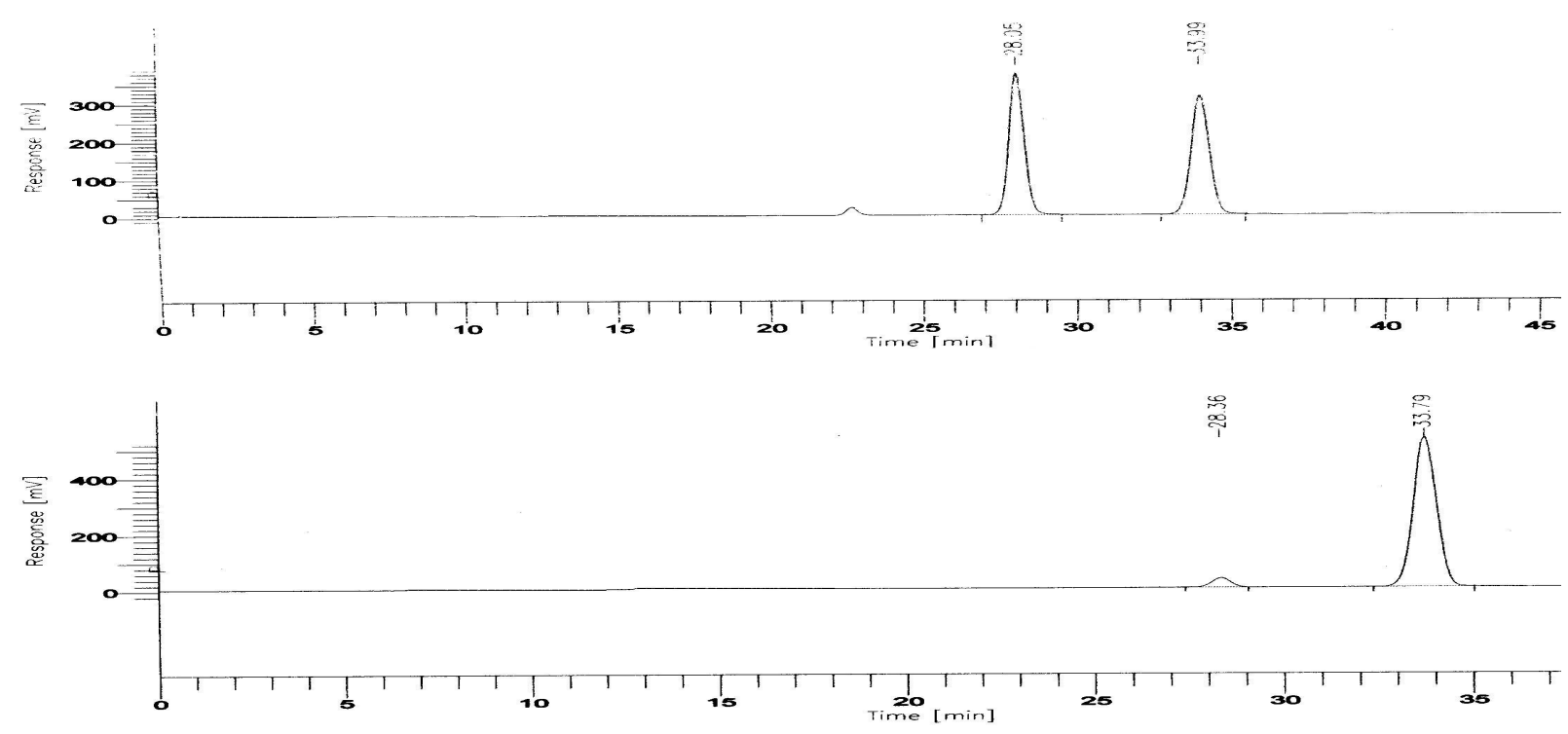

HPLC graph for racemic and enanrioenriched compound (Table 3, Entry 6).

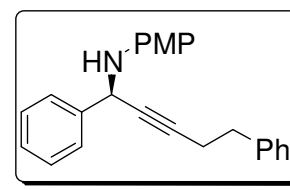

(R)-(1, 5-Diphenyl-pent-2-ynyl)-(4-methoxy-phenyl)-amine (Table

5, Entry 5). $84 \%$ ee; isolated in $86 \%$ yield as yellow gel; $R_{\mathrm{f}}=0.48$ (EtOAc: petroleum ether = 1:9); HPLC conditions: Diacel chiralpak AD-H (4.6 cm I.D. x $25 \mathrm{~cm})$, 95:5 hexanes: ${ }^{i}-\mathrm{PrOH}, 1.0 \mathrm{~mL} / \mathrm{min}$ flow rate, $\lambda=254 \mathrm{~nm}, \mathrm{~T}_{\mathrm{R}}$ (major) $=19.84 \mathrm{~min}$ and $\mathrm{T}_{\mathrm{R}}$ $($ minor $)=15.34 \mathrm{~min}$; IR (thin film): 3389, 3058, 3029, 2929, 2857, 1728, 1599, 1511, $1543,1238,1178,1115,1035,821,741,700 \mathrm{~cm}-1 ;{ }^{1} \mathrm{H} \mathrm{NMR}\left(\mathrm{CDCl}_{3}, 400 \mathrm{MHz}\right): \delta 7.49$ $(\mathrm{d}, J=7.32 \mathrm{~Hz}, 2 \mathrm{H}), 7.14-7.35(\mathrm{~m}, 8 \mathrm{H}), 6.70(\mathrm{dd}, J=47.84,7.84 \mathrm{~Hz}, 4 \mathrm{H}), 5.12(\mathrm{~s}, 1 \mathrm{H})$, $3.73(\mathrm{~s}, 3 \mathrm{H}), 2.78(\mathrm{t}, J=7.32 \mathrm{~Hz}, 2 \mathrm{H}), 2.50(\mathrm{t}, J=7.32 \mathrm{~Hz}, 2 \mathrm{H}) ;{ }^{13} \mathrm{C}\left(\mathrm{CDCl}_{3}, 100 \mathrm{MHz}\right)$ : $\delta 140.8,140.5,140.3,128.5,128.4,128.3,127.7,127.2,126.2,115.6,114.5,84.6,80.4$, 55.6, 51.1, 34.9, 20.9; Anal. cacld. for $\mathrm{C}_{24} \mathrm{H}_{23} \mathrm{NO}$ : C, 84.42; H, 6.79; N, 4.10. Found: C, 84.68; H, 6.85; N, 4.07. Optical Rotation: $[\alpha]^{25}+68.7\left(\mathrm{c} 0.7, \mathrm{CHCl}_{3}\right)$. 


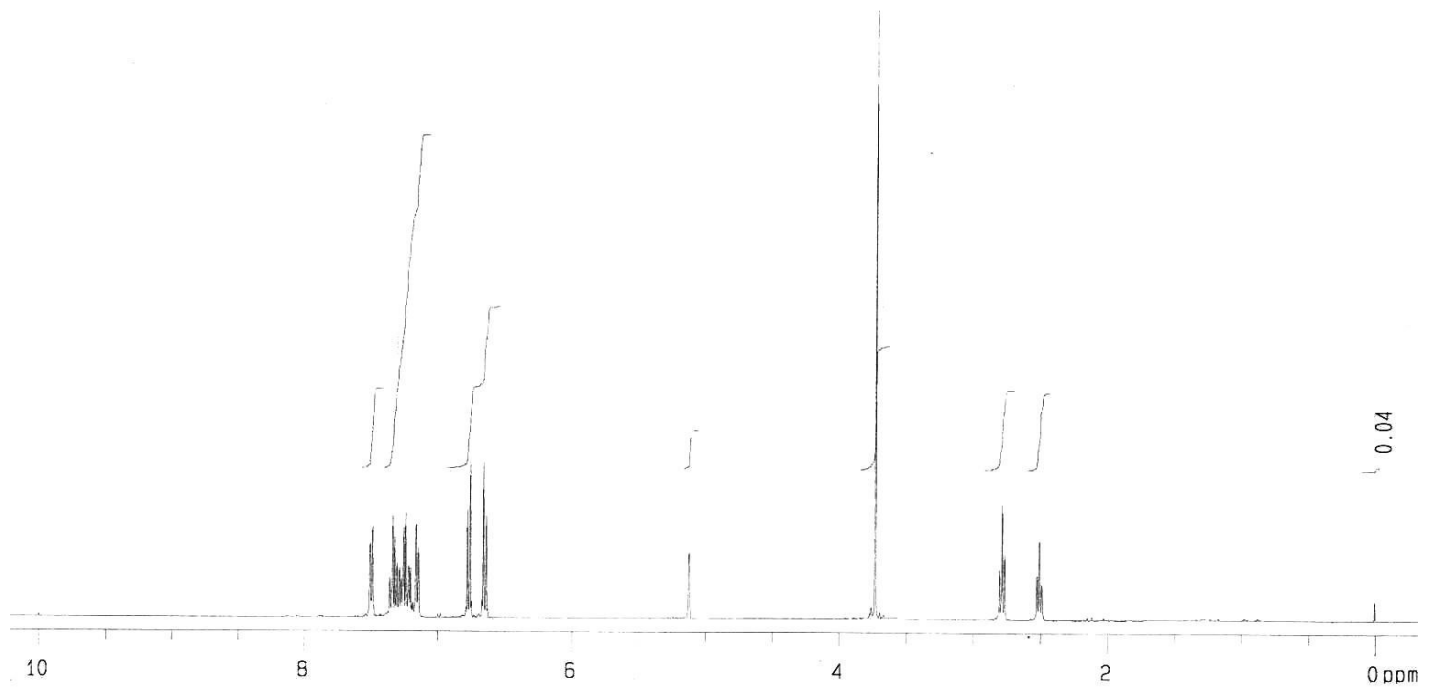

$400 \mathrm{MHz}{ }^{1} \mathrm{H}$ NMR Spectra for compound (Table 5, Entry 5).

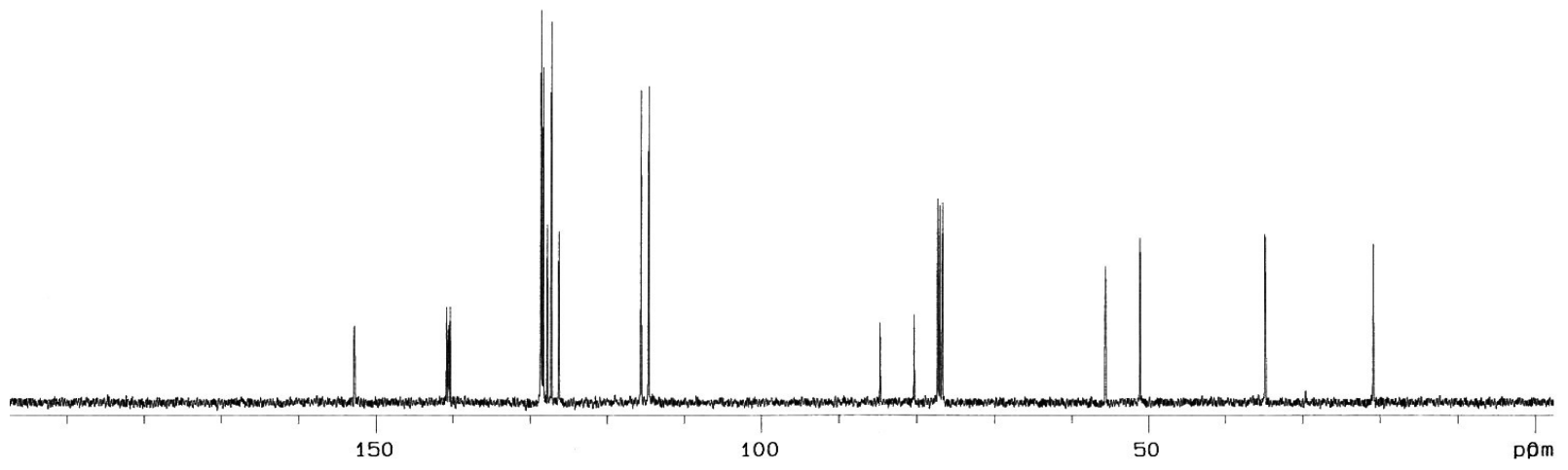

$100 \mathrm{MHz}{ }^{13} \mathrm{C}$ NMR Spectra for compound (Table 5, Entry 5). 

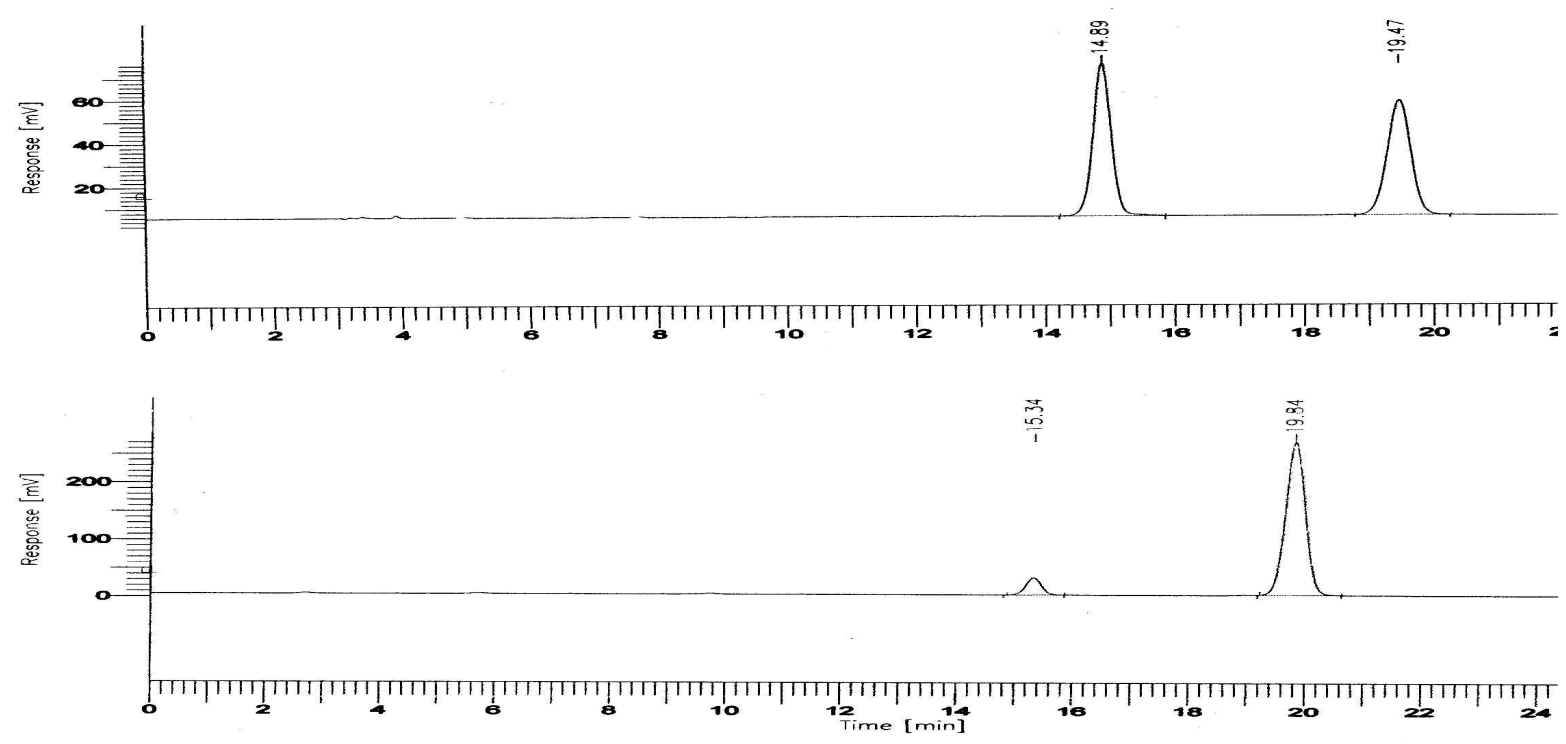

HPLC graph for racemic and enanrioenriched compound (Table 5, Entry 5).

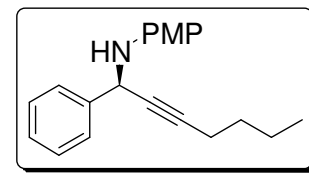

(R)-(1-Phenyl-hept-2-ynyl)-(4-methoxy-phenyl)-amine (Table 5,

Entry 6). $87 \%$ ee; isolated in $67 \%$ yield as yellow gel; $R_{\mathrm{f}}=0.59$ (EtOAc: petroleum ether = 1:9); HPLC conditions: Diacel chiralpak AD-H (4.6 cm I.D. x $25 \mathrm{~cm}), 95: 5$ hexanes: ${ }^{i}-$ $\mathrm{PrOH}, 0.5 \mathrm{~mL} / \mathrm{min}$ flow rate, $\lambda=254 \mathrm{~nm}, \mathrm{~T}_{\mathrm{R}}($ major $)=19.69 \mathrm{~min}$ and $\mathrm{T}_{\mathrm{R}}$ (minor) $=$ 16.81 min; IR (thin film): 3386, 3062, 2955, 2865, 1593, 1512, 1466, 1239, 1181, 1121, 1038, 820, 756, $703 \mathrm{~cm}-1$; ${ }^{1} \mathrm{H}$ NMR $\left(\mathrm{CDCl}_{3}, 400 \mathrm{MHz}\right): \delta 7.56(\mathrm{~d}, J=7.32 \mathrm{~Hz}, 2 \mathrm{H})$, 7.27-7.37 (m, 3H), $6.70(\mathrm{dd}, J=35.50,8.80 \mathrm{~Hz}, 4 \mathrm{H}), 5.15(\mathrm{~s}, 1 \mathrm{H}), 3.73(\mathrm{~s}, 3 \mathrm{H}), 2.20(\mathrm{dt}$, $J=7.08,1.96 \mathrm{~Hz}, 2 \mathrm{H}), 1.42-1.49(\mathrm{~m}, 2 \mathrm{H}), 1.34-1.39(\mathrm{~m}, 2 \mathrm{H}), 0.86(\mathrm{t}, J=7.32 \mathrm{~Hz}, 3 \mathrm{H})$; ${ }^{13} \mathrm{C}\left(\mathrm{CDCl}_{3}, 100 \mathrm{MHz}\right): \delta 152.7,140.8,140.5,128.5,127.7,127.1,115.7,114.5,85.6$, 79.4, 55.6, 51.2, 30.7, 21.8, 18.4, 13.6; Anal. cacld. for $\mathrm{C}_{20} \mathrm{H}_{23} \mathrm{NO}$ : C, 81.87; H, 7.90; N, 4.77. Found: C, 82.01; H, 7.97; N, 4.65. Optical Rotation: $[\alpha]^{25}+64.0$ (c 1.6, $\mathrm{CHCl}_{3}$ ). 

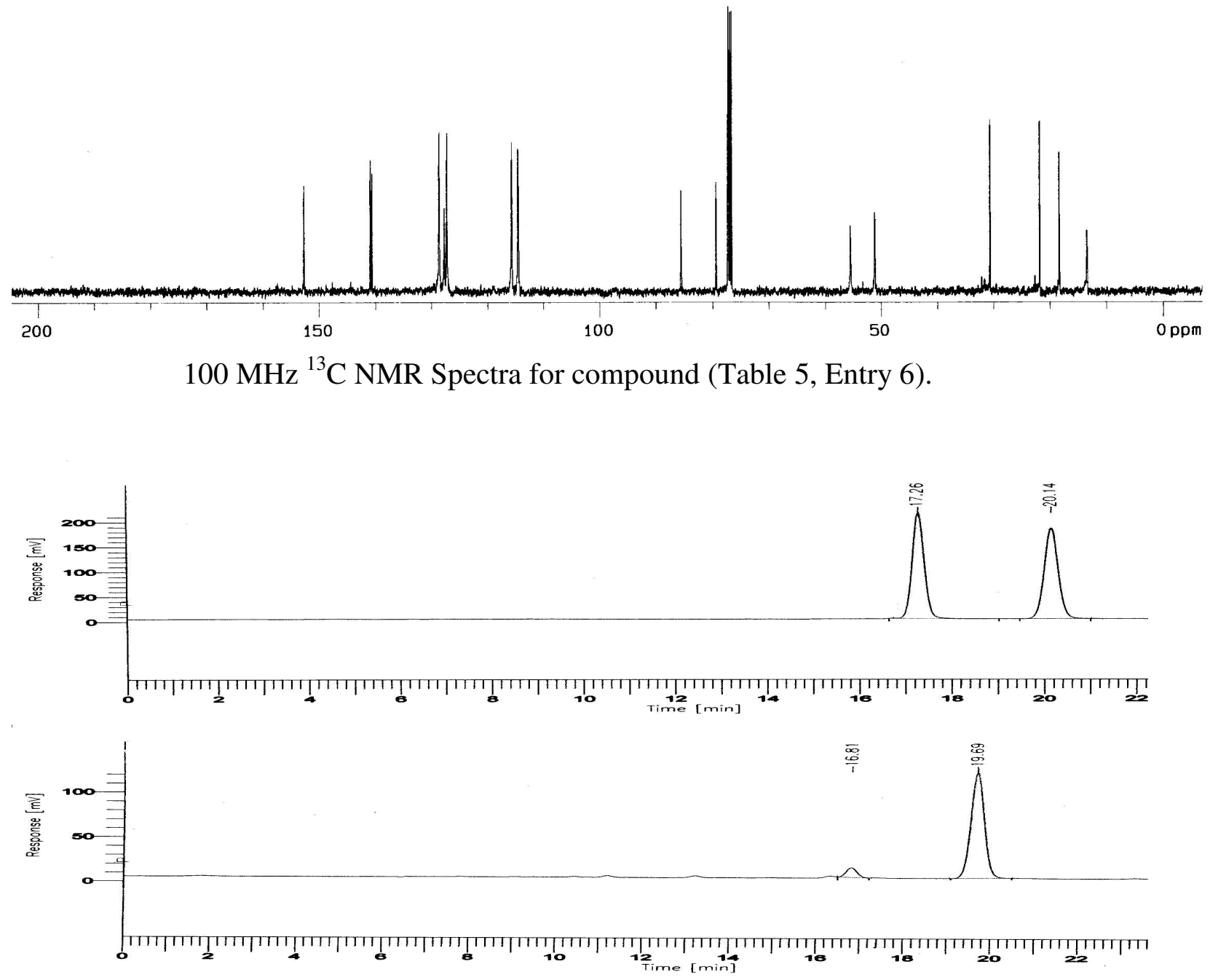

HPLC graph for racemic and enanrioenriched compound (Table 5, Entry 6).

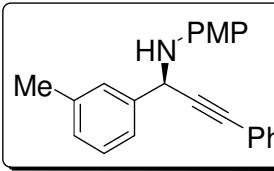

(R)-\{1-(3-Methyl-phenyl)-3-phenyl-prop-2-ynyl\}-(4-methoxy-

phenyl)-amine (Table 4, Entry 1). $92 \%$ ee; isolated in $99 \%$ yield as yellow gel; $R_{f}=$ 
0.48 (EtOAc: petroleum ether = 1:9); HPLC conditions: Diacel chiralpak AD-H $(4.6 \mathrm{~cm}$ I.D. $x 25 \mathrm{~cm}$ ), 95:5 hexanes: ${ }^{i}-\mathrm{PrOH}, 0.5 \mathrm{~mL} / \mathrm{min}$ flow rate, $\lambda=254 \mathrm{~nm}, \mathrm{~T}_{\mathrm{R}}$ (major) $=$ $28.24 \mathrm{~min}$ and $\mathrm{T}_{\mathrm{R}}$ (minor) $=25.41 \mathrm{~min}$; IR (thin film): 3368, 2926, 2856, 1735, 1601, 1510, 1459, 1240, 1172, 1033, 821, 738, $697 \mathrm{~cm}-1$; ${ }^{1} \mathrm{H}$ NMR $\left(\mathrm{CDCl}_{3}, 400 \mathrm{MHz}\right): \delta 7.22-$ 7.45 (m, 9H), 7.13-7.14 (m, 1H), 6.74-6.80 (m, 3H), 5.35 (s, 1H), 3.73 (s, 3H), 2.37 (s, $3 \mathrm{H}) ;{ }^{13} \mathrm{C}\left(\mathrm{CDCl}_{3}, 100 \mathrm{MHz}\right): \delta 152.9,140.7,139.8,138.4,131.7,128.7,128.6,128.1$, 128.0, 124.3, 122.8 (d), 115.8, 114.6, 113.6, 88.9, 84.9, 55.6, 51.7, 21.4; Anal. cacld. for $\mathrm{C}_{23} \mathrm{H}_{21} \mathrm{NO}$ : C, 84.37; H, 6.46; N, 4.28. Found: C, 84.63; H, 6.55; N, 4.31. Optical Rotation: $[\alpha]^{25}+104.0\left(\mathrm{c} 1.2, \mathrm{CHCl}_{3}\right)$.

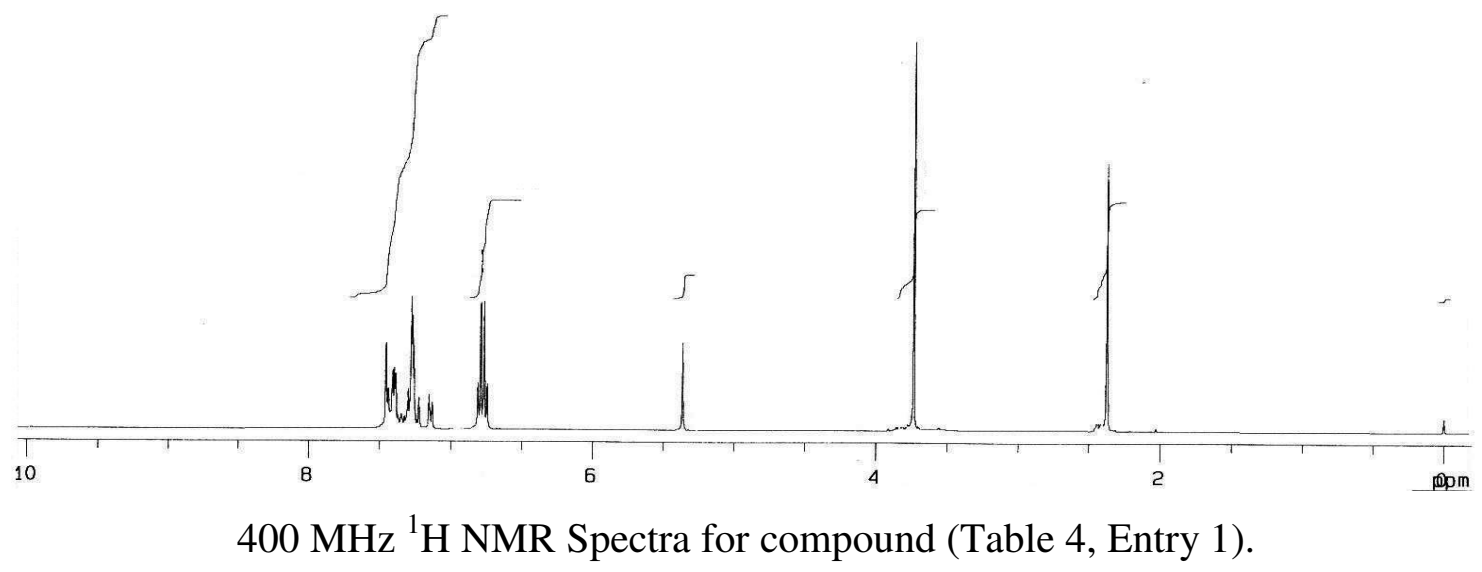




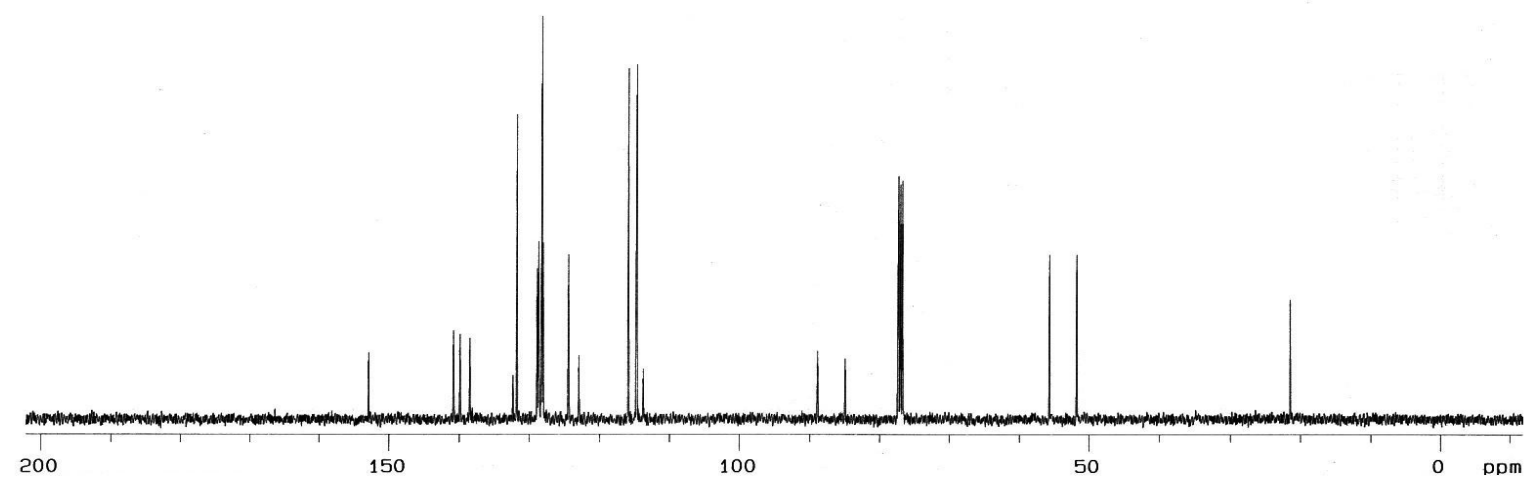

$100 \mathrm{MHz}{ }^{13} \mathrm{C}$ NMR Spectra for compound (Table 4, Entry 1).
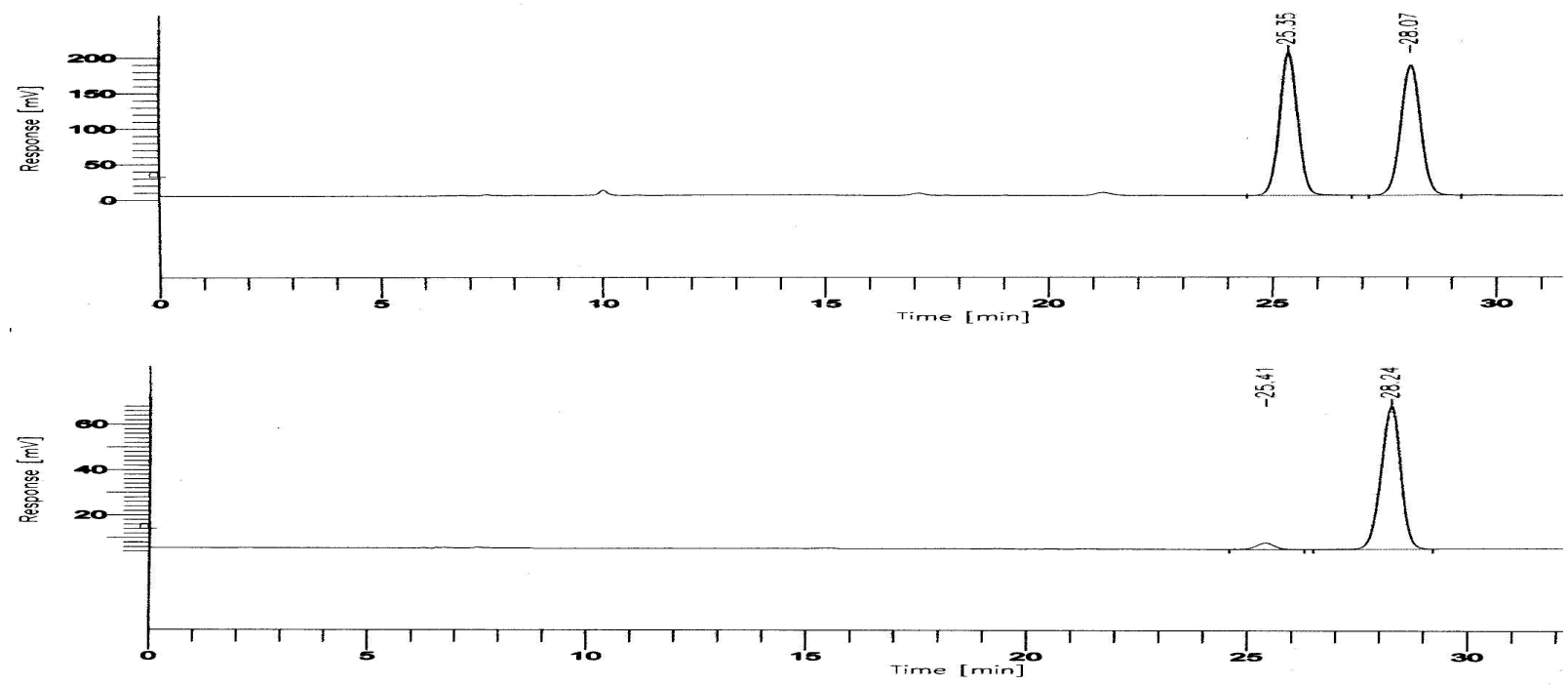

HPLC graph for racemic and enanrioenriched compound (Table 4, Entry 1).

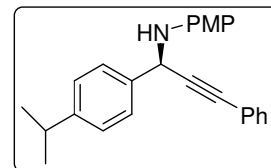

(R)-\{1-(4-Isopropyl-phenyl)-3-phenyl-prop-2-ynyl\}-(4-methoxy-

phenyl)-amine (Table 4, Entry 2). $83 \%$ ee; isolated in $87 \%$ yield as yellow gel; $R_{f}=$ 0.60 (EtOAc: petroleum ether = 1:9); HPLC conditions: Diacel chiralpak AD-H $(4.6 \mathrm{~cm}$ I.D. $x 25 \mathrm{~cm}$ ), 95:5 hexanes: ${ }^{i}-\mathrm{PrOH}, 1.0 \mathrm{~mL} / \mathrm{min}$ flow rate, $\lambda=254 \mathrm{~nm}, \mathrm{~T}_{\mathrm{R}}$ (major) $=$ 
$12.10 \mathrm{~min}$ and $\mathrm{T}_{\mathrm{R}}$ (minor) $=14.65 \mathrm{~min}$; $\mathrm{IR}$ (thin film): 3386, 2958, 2927, 2862, 1735, 1600, 1511, 1461, 1238, 1113, 1036, 821, $751 \mathrm{~cm}-1 ;{ }^{1} \mathrm{H}$ NMR (CDCl 3 , $\left.400 \mathrm{MHz}\right): \delta 7.57$ (d, $J=8.08 \mathrm{~Hz}, 2 \mathrm{H}), 7.38-7.40(\mathrm{~m}, 2 \mathrm{H}), 7.22-7.28(\mathrm{~m}, 5 \mathrm{H}), 6.75-6.82(\mathrm{~m}, 4 \mathrm{H}), 5.37$ (s, $1 \mathrm{H}), 3.74(\mathrm{~s}, 1 \mathrm{H}), 2.92(\mathrm{~m}, 1 \mathrm{H}), 1.28(\mathrm{~d}, J=7.08 \mathrm{~Hz}, 6 \mathrm{H}) ;{ }^{13} \mathrm{C}\left(\mathrm{CDCl}_{3}, 100 \mathrm{MHz}\right): \delta$ 152.8, 148.7, 140.8, 137.2, 131.7, 128.1, 127.3, 126.8, 122.9, 115.8, 114.6, 88.9, 84.8, 55.6, 51.4, 33.8, 23.9; Anal. cacld. for $\mathrm{C}_{25} \mathrm{H}_{25} \mathrm{NO}$ : C, 84.47; H, 7.09; N, 3.94. Found: $\mathrm{C}$, 84.75; H, 7.16; N, 4.05. Optical Rotation: $[\alpha]^{25}{ }_{\mathrm{D}}+93.1$ (c 1.3, $\left.\mathrm{CHCl}_{3}\right)$.
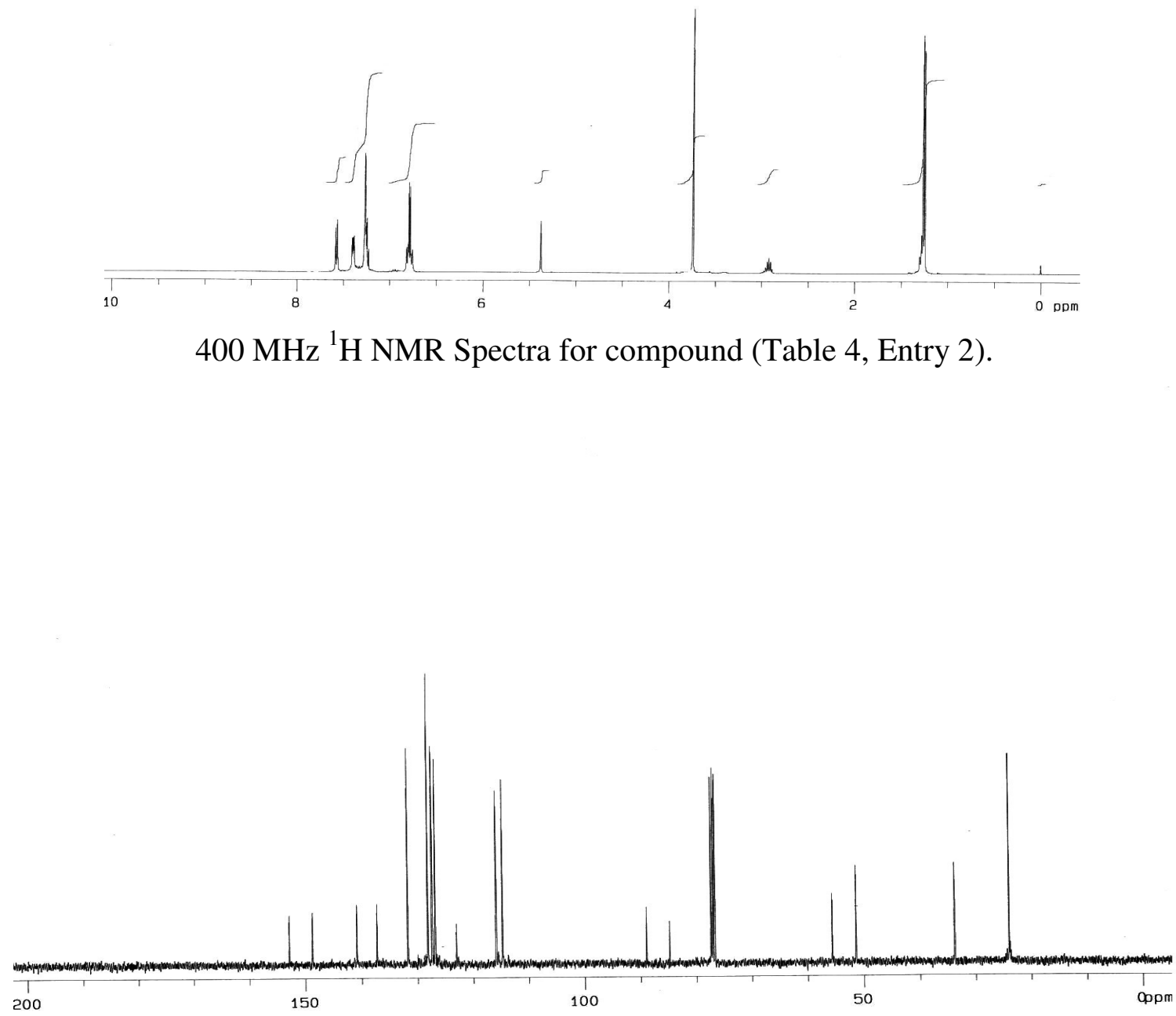

$100 \mathrm{MHz}{ }^{13} \mathrm{C}$ NMR Spectra for compound (Table 4, Entry 2). 

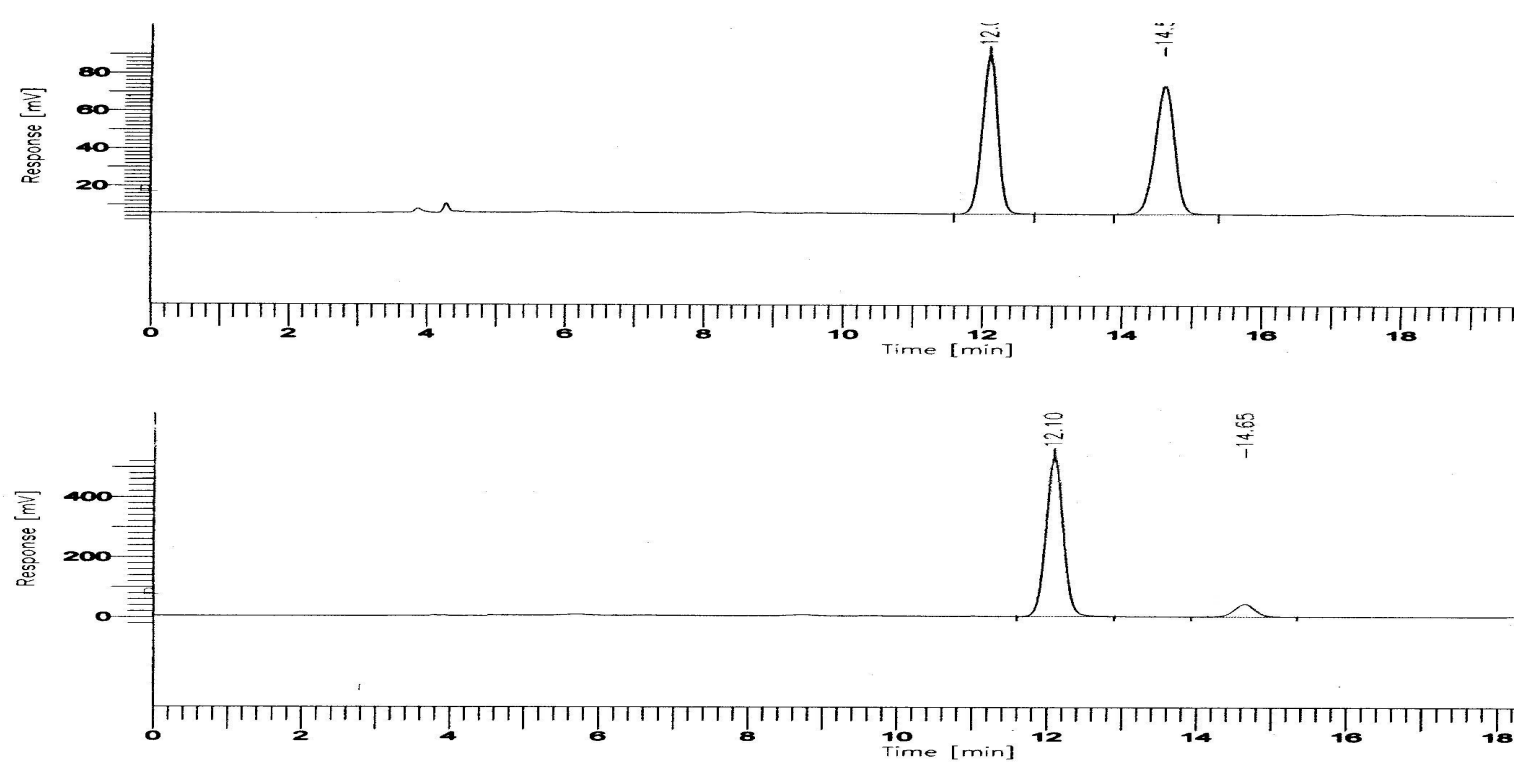

HPLC graph for racemic and enanrioenriched compound (Table 4, Entry 2).

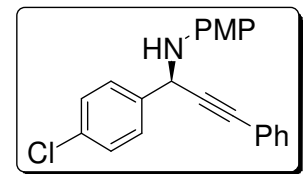

\section{(R)-\{1-(4-Chloro-phenyl)-3-phenyl-prop-2-ynyl\}-(4-methoxy-}

phenyl)-amine (Table 4, Entry 3). $90 \%$ ee; isolated in $91 \%$ yield as yellow gel; $\mathrm{R}_{\mathrm{f}}=$ 0.48 (EtOAc: petroleum ether = 1:9); HPLC conditions: Diacel chiralpak AD-H $(4.6 \mathrm{~cm}$ I.D. $x 25 \mathrm{~cm}$ ), 95:5 hexanes: ${ }^{i}-\mathrm{PrOH}, 1.0 \mathrm{~mL} / \mathrm{min}$ flow rate, $\lambda=254 \mathrm{~nm}, \mathrm{~T}_{\mathrm{R}}$ (major) $=$ 22.20 min and $\mathrm{T}_{\mathrm{R}}$ (minor) = $20.17 \mathrm{~min}$; IR (thin film): 3366 (br), 3056 (br), 2924 (br), 2833 (br), 1593, 1510, 1466, 1420, 1237, 1179, 1071, 1034, 820, 789, 755, $687 \mathrm{~cm}-1 ;{ }^{1} \mathrm{H}$ NMR $\left(\mathrm{CDCl}_{3}, 400 \mathrm{MHz}\right): \delta 7.56(\mathrm{~d}, J=8.28 \mathrm{~Hz}, 2 \mathrm{H}), 7.21-7.48(\mathrm{~m}, 7 \mathrm{H}), 6.75(\mathrm{dd}, J=$ 31.36, 8.76 Hz, 4H), $5.36(\mathrm{~s}, 1 \mathrm{H}), 3.70(\mathrm{~s}, 3 \mathrm{H})$; Anal. cacld. for $\mathrm{C}_{22} \mathrm{H}_{18} \mathrm{ClNO}$ : C, 75.97; H, 5.22; N, 4.03. Found: C, 76.20; H, 5.25; N, 4.12. Optical Rotation: $[\alpha]^{25}+76.2$ (c $\left.1.8, \mathrm{CHCl}_{3}\right)$. 


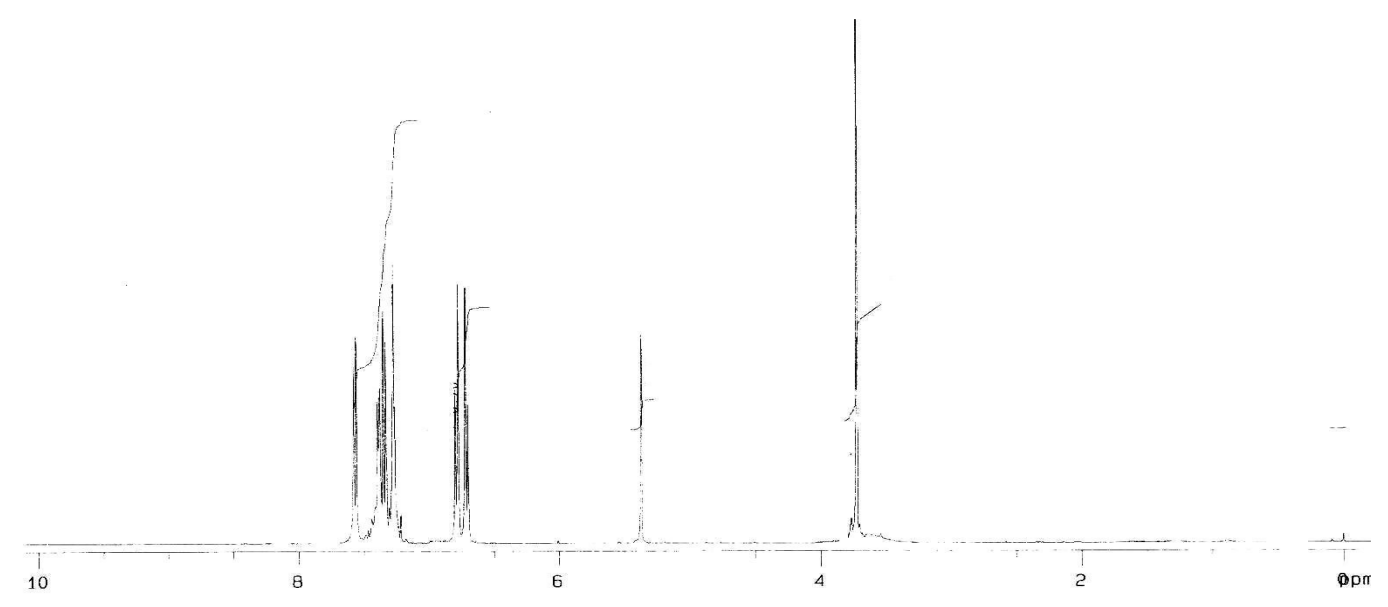

$400 \mathrm{MHz}{ }^{1} \mathrm{H}$ NMR Spectra for compound (Table 4, Entry 3).
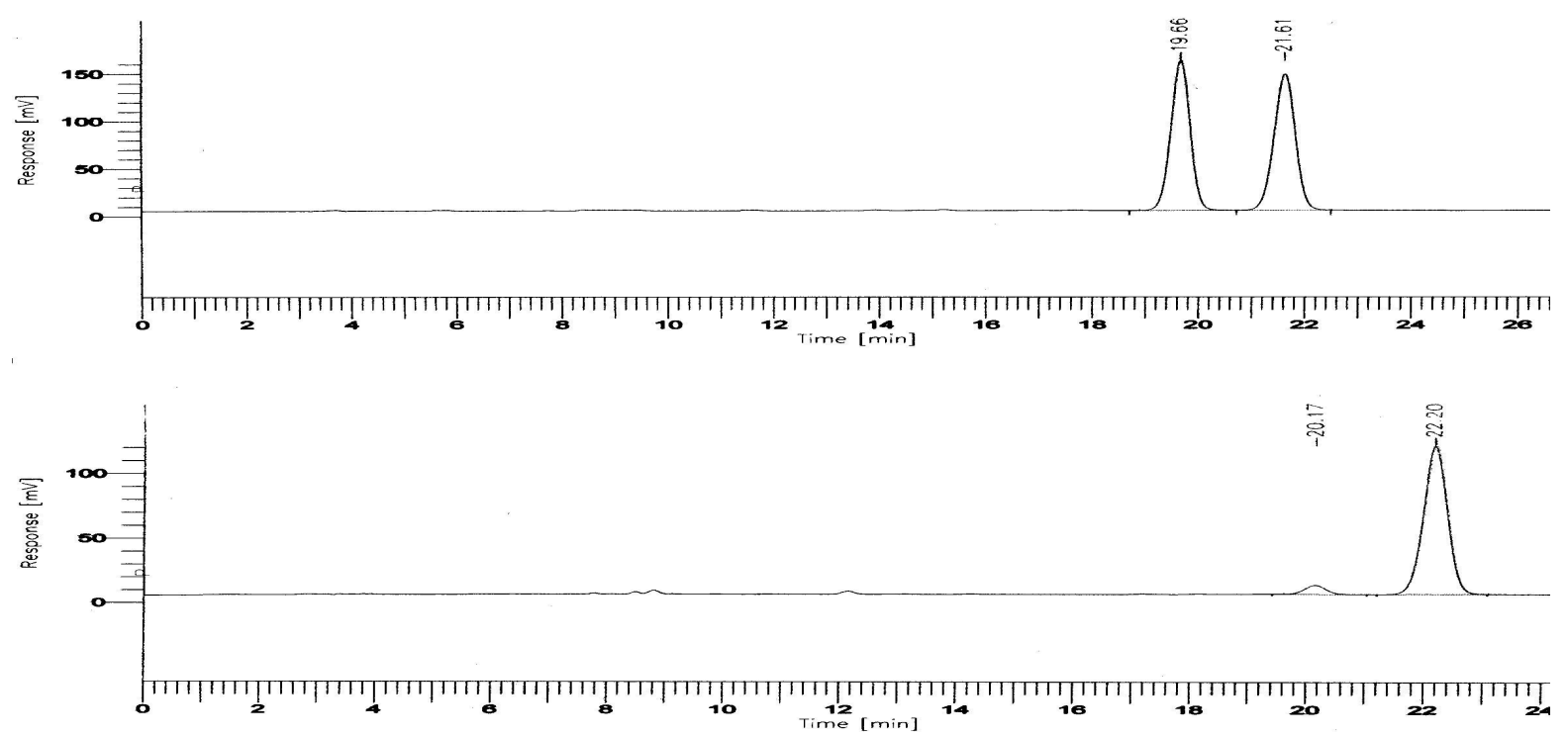

HPLC graph for racemic and enanrioenriched compound (Table 4, Entry 3).<smiles>CN(C)C(=Cc1cccc(F)c1)Nc1ccccc1</smiles>

\section{(R)-\{1-(3-Fluoro-phenyl)-3-phenyl-prop-2-ynyl\}-(4-methoxy-}

phenyl)-amine (Table 4, Entry 4). 85\% ee; isolated in $90 \%$ yield as yellow gel; $R_{f}=$ 0.44 (EtOAc: petroleum ether = 1:9); HPLC conditions: Diacel chiralpak AD-H $(4.6 \mathrm{~cm}$ I.D. $x 25 \mathrm{~cm}$ ), 95:5 hexanes: ${ }^{i}-\mathrm{PrOH}, 1.0 \mathrm{~mL} / \mathrm{min}$ flow rate, $\lambda=254 \mathrm{~nm}, \mathrm{~T}_{\mathrm{R}}$ (major) $=$ 
17.17 min and $\mathrm{T}_{\mathrm{R}}$ (minor) = $13.86 \mathrm{~min}$; IR (thin film): 3368 (br), 2926, 1591, 1510, 1444, $1239,1176,1125,1034,880,822,784,754,689 \mathrm{~cm}-1 ;{ }^{1} \mathrm{H} \mathrm{NMR}\left(\mathrm{CDCl}_{3}, 400 \mathrm{MHz}\right): \delta$ 7.25-7.44 (m, 8H), 6.96-7.03 (m, 1H), 6.70-6.80 (m, 4H), $5.39(\mathrm{~s}, 1 \mathrm{H}), 3.73(\mathrm{~s}, 3 \mathrm{H})$ : Anal. cacld. for $\mathrm{C}_{22} \mathrm{H}_{18}$ FNO: C, 79.74; H, 5.47; N, 4.23. Found: C, 79.91; H, 5.41; N, 4.31. Optical Rotation: $[\alpha]^{25}+104.6\left(\mathrm{c} 1.0, \mathrm{CHCl}_{3}\right)$.
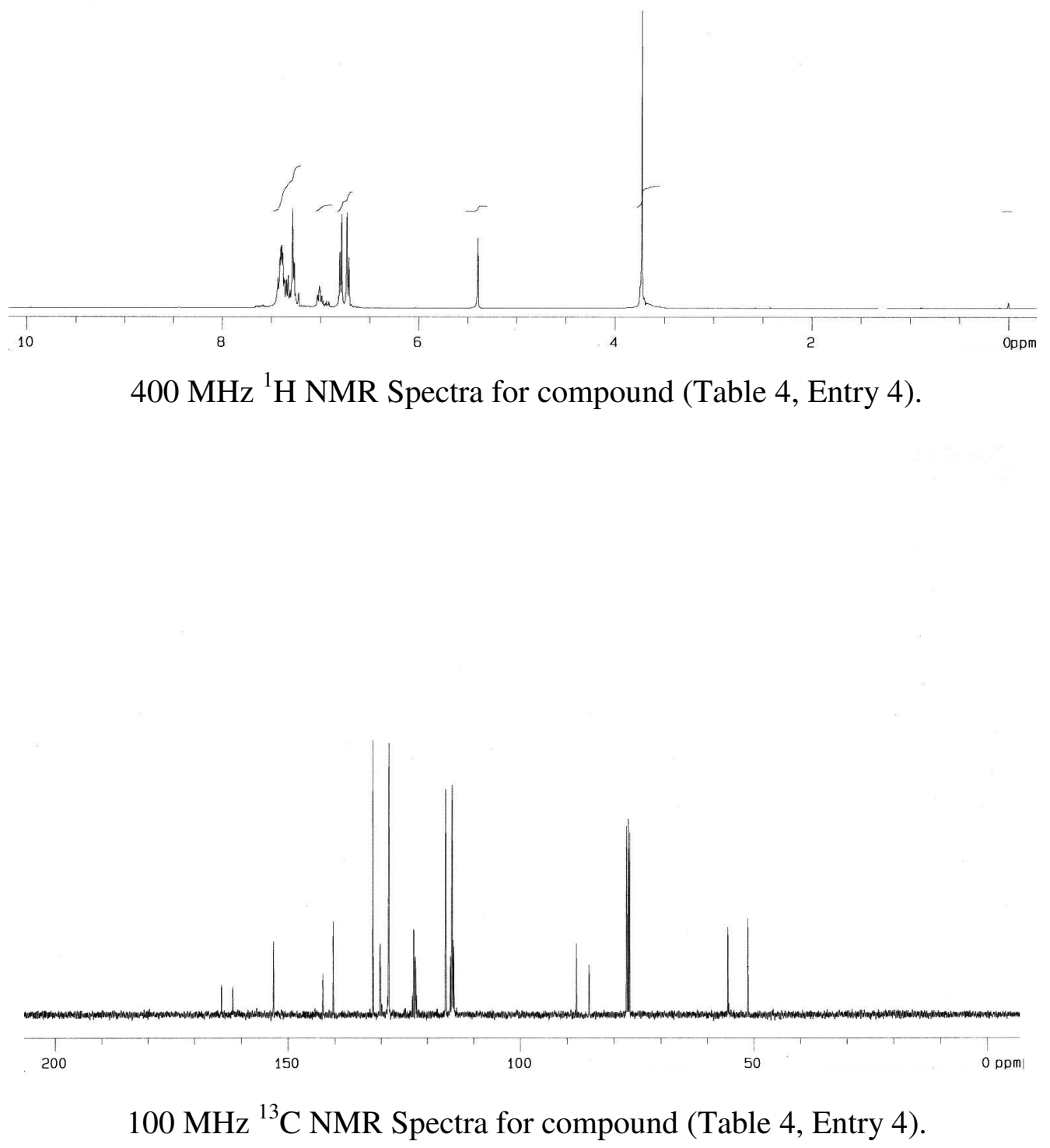

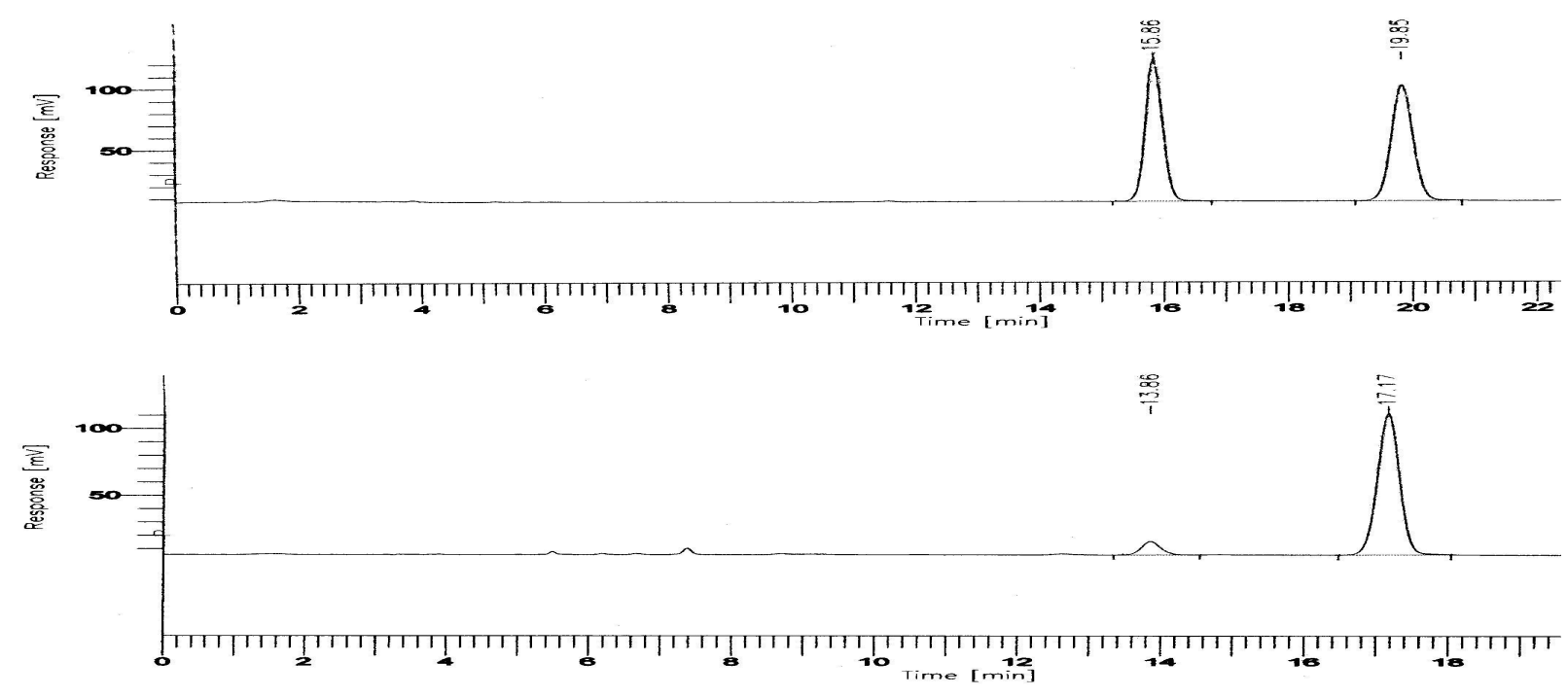

HPLC graph for racemic and enanrioenriched compound (Table 4, Entry 4).

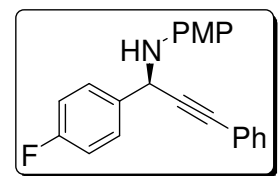

(R)-\{1-(4-Fluoro-phenyl)-3-phenyl-prop-2-ynyl\}-(4-methoxy-

phenyl)-amine (Table 4, Entry 5). $91 \%$ ee; isolated in $95 \%$ yield as yellow gel; $R_{f}=$ 0.52 (EtOAc: petroleum ether = 1:9); HPLC conditions: Diacel chiralpak AD-H $(4.6 \mathrm{~cm}$ I.D. $x 25 \mathrm{~cm}$ ), 95:5 hexanes: ${ }^{i}-\mathrm{PrOH}, 1.0 \mathrm{~mL} / \mathrm{min}$ flow rate, $\lambda=254 \mathrm{~nm}, \mathrm{~T}_{\mathrm{R}}$ (major) $=$ 18.83 min and $\mathrm{T}_{\mathrm{R}}($ minor $)=16.08 \mathrm{~min}$; IR (thin film): 3369, 2925, 1592, 1511, 1445 , 1240, 1035, 881, 784, 689cm-1; ${ }^{1} \mathrm{H}$ NMR $\left(\mathrm{CDCl}_{3}, 400 \mathrm{MHz}\right): \delta 7.59-7.63$ (m, 2H), 7.37$7.40(\mathrm{~m}, 2 \mathrm{H}), 7.23-7.30(\mathrm{~m}, 3 \mathrm{H}), 7.03-7.08(\mathrm{~m}, 2 \mathrm{H}), 6.71-6.81(\mathrm{~m}, 4 \mathrm{H}), 5.37(\mathrm{~s}, 1 \mathrm{H}), 3.72$ $(\mathrm{s}, 3 \mathrm{H}) ;{ }^{13} \mathrm{C}\left(\mathrm{CDCl}_{3}, 100 \mathrm{MHz}\right): \delta 163.6,161.1,153.0,140.4,135.6,131.6,129.0,128.9$, 128.3, 128.2, 122.6, 115.9, 115.5, 115.3, 114.6, 88.4, 85.3, 55.6, 51.0; Anal. cacld. for $\mathrm{C}_{22} \mathrm{H}_{18} \mathrm{FNO}$ : C, 79.74; H, 5.47; N, 4.23. Found: C, 80.01; H, 6.54; N, 4.18. Optical Rotation: $[\alpha]^{25}+117.6\left(\mathrm{c} 1.8, \mathrm{CHCl}_{3}\right)$. 

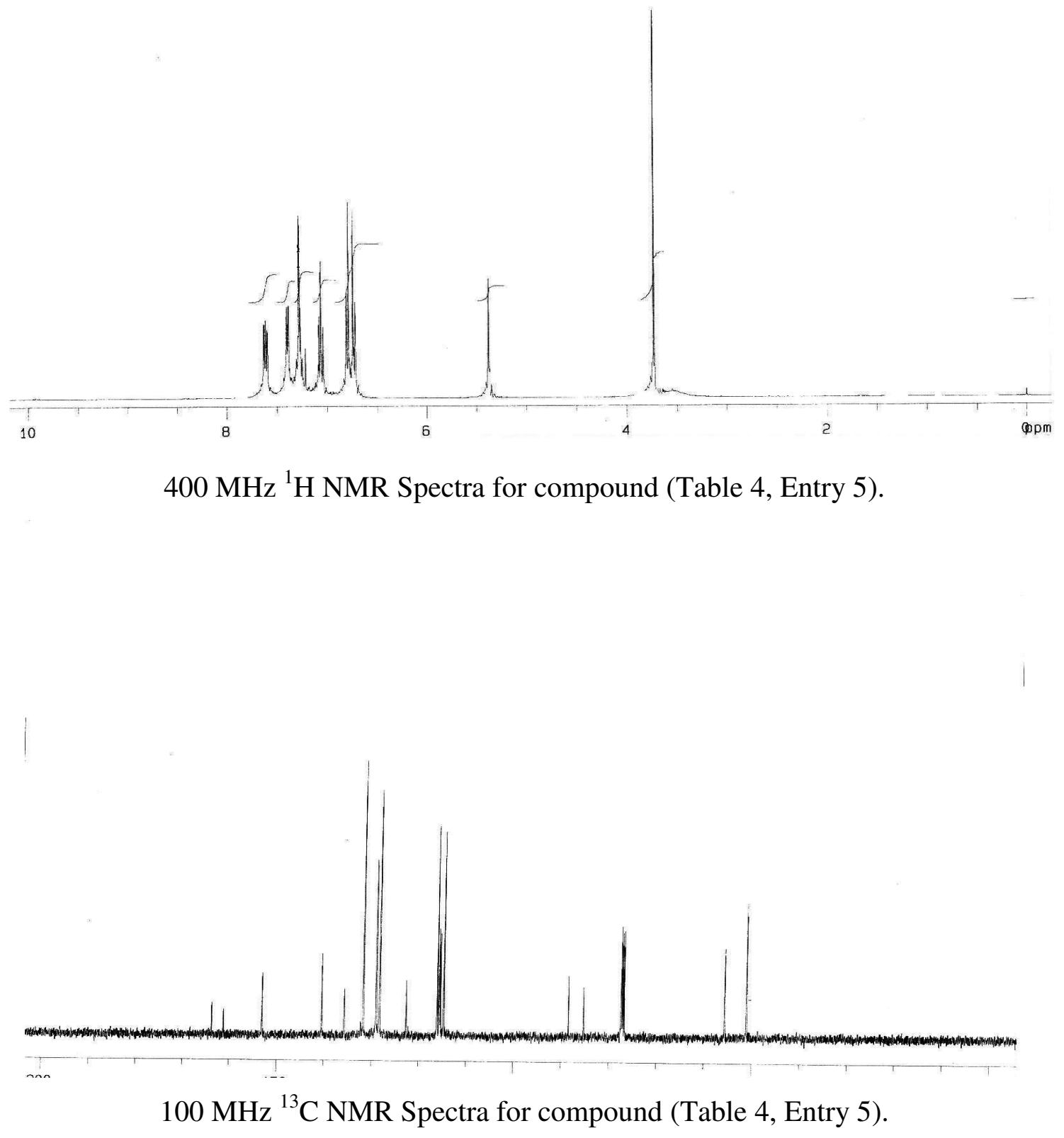

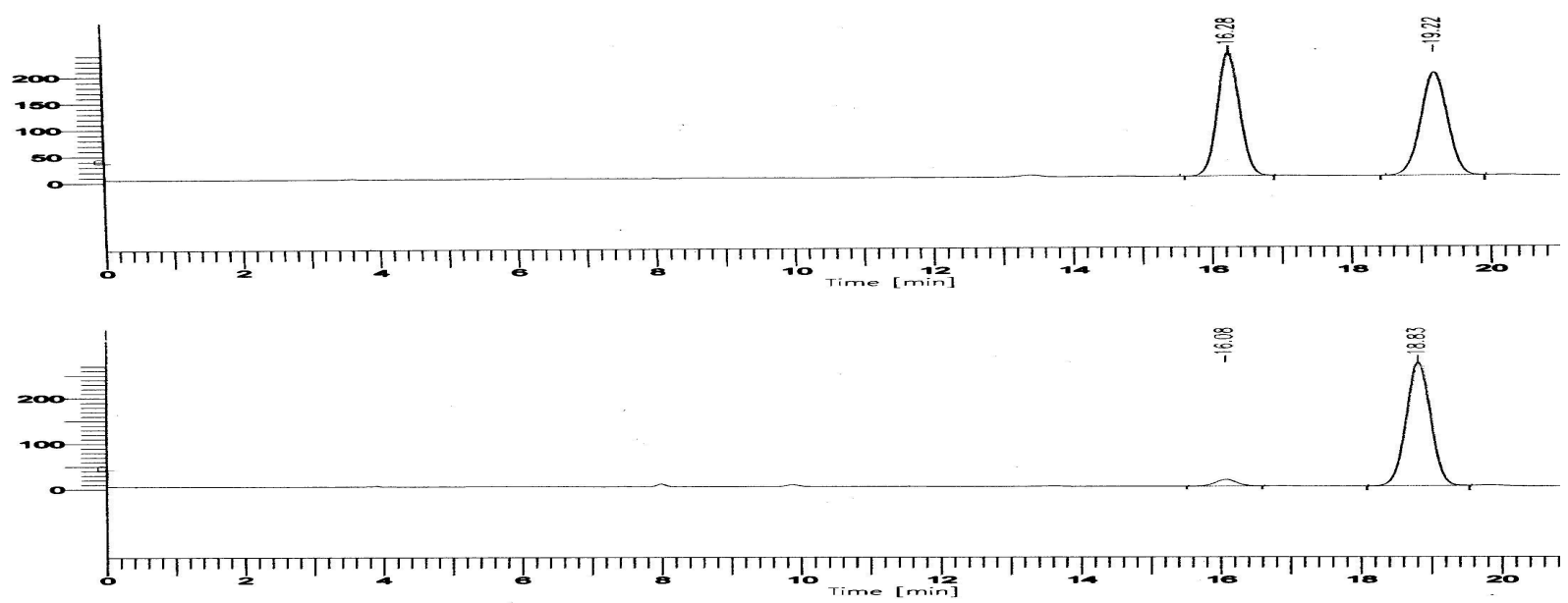

HPLC graph for racemic and enanrioenriched compound (Table 4, Entry 5).

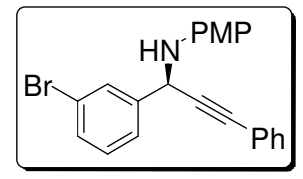

(R)-\{1-(3-Bromo-phenyl)-3-phenyl-prop-2-ynyl\}-(4-methoxy-

phenyl)-amine (Table 4, Entry 6). $80 \%$ ee; isolated in $96 \%$ yield as yellow gel; $\mathrm{R}_{\mathrm{f}}=$ 0.56 (EtOAc: petroleum ether = 1:9); HPLC conditions: Diacel chiralpak AD-H $(4.6 \mathrm{~cm}$ I.D. $\mathrm{x} 25 \mathrm{~cm}$ ), 95:5 hexanes: ${ }^{i}-\mathrm{PrOH}, 0.5 \mathrm{~mL} / \mathrm{min}$ flow rate, $\lambda=254 \mathrm{~nm}, \mathrm{~T}_{\mathrm{R}}$ (major) $=$ $35.29 \mathrm{~min}$ and $\mathrm{T}_{\mathrm{R}}$ (minor) $=29.48 \mathrm{~min}$; IR (thin film): 3353, 2925, 2855, 1594, 1511, 1466, 1238, 1178, 1070, 1034, 820, 755, $690 \mathrm{~cm}-1 ;{ }^{1} \mathrm{H}$ NMR $\left(\mathrm{CDCl}_{3}, 400 \mathrm{MHz}\right): \delta 7.80$ (s, 1H), 7.55-7.57 (m, 1H), 7.42-7.44 (m, 1H), 7.37-7.39 (m, 2H), 7.20-7.28 (m, 4H), 6.69-6.79 (m, 4H), $5.35(\mathrm{~s}, 1 \mathrm{H}), 3.71(\mathrm{~s}, 3 \mathrm{H}) ;{ }^{13} \mathrm{C}\left(\mathrm{CDCl}_{3}, 100 \mathrm{MHz}\right): \delta 153.0,142.2$, $140.2,131.7,131.0,130.3,130.2,128.4,128.2$, 125.9, 122.7, 122.4, 115.9, 114.6, 87.9, 85.5, 55.5, 51.2; Anal. cacld. for $\mathrm{C}_{22} \mathrm{H}_{18} \mathrm{BrNO}$ : C, 67.36; H, 4.62; N, 3.57. Found: C, 67.59; H, 4.69; N, 3.59. Optical Rotation: $[\alpha]^{25}+68.1$ (c 2.1, $\mathrm{CHCl}_{3}$ ). 

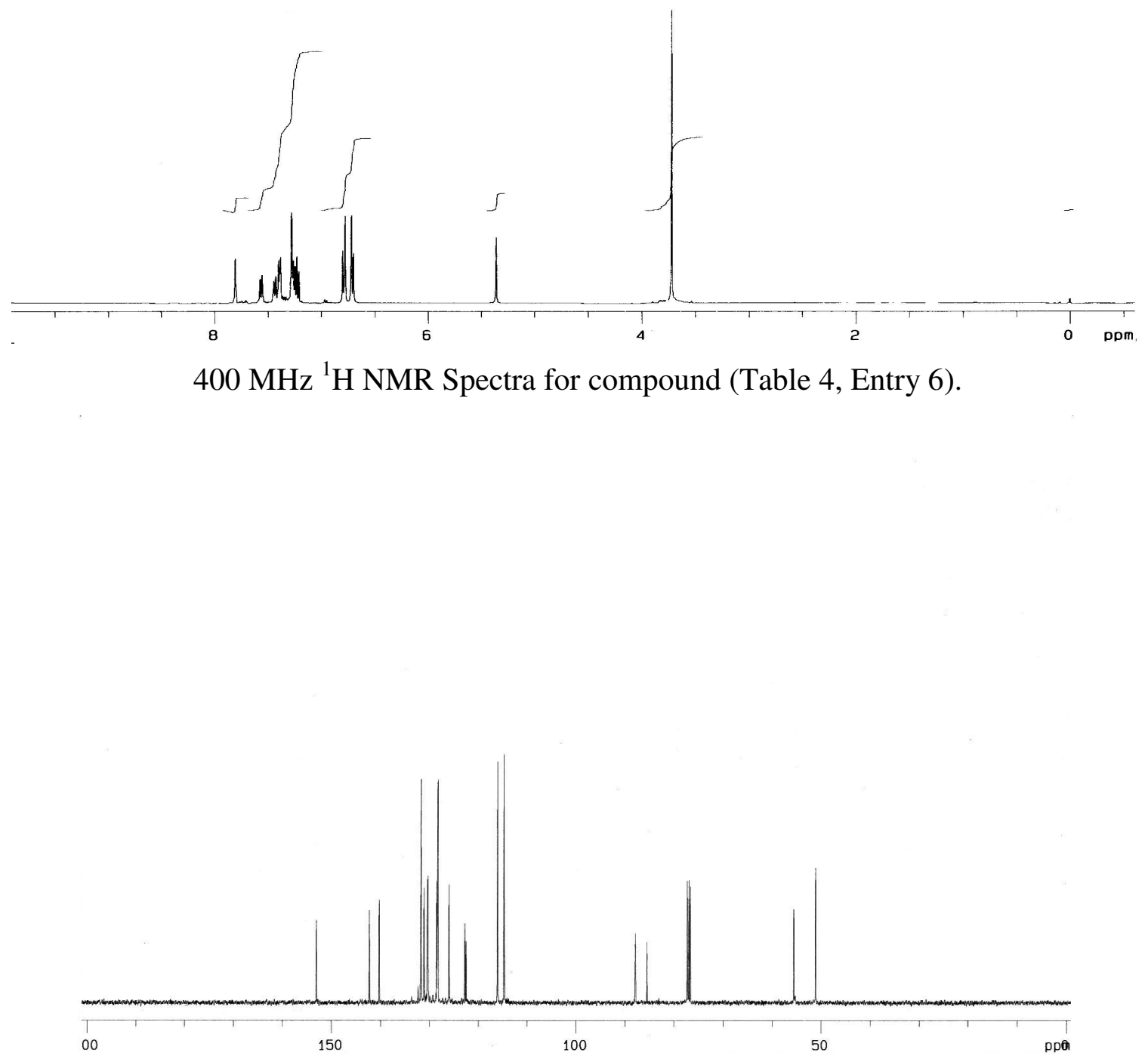

$100 \mathrm{MHz}{ }^{13} \mathrm{C}$ NMR Spectra for compound (Table 4, Entry 6). 

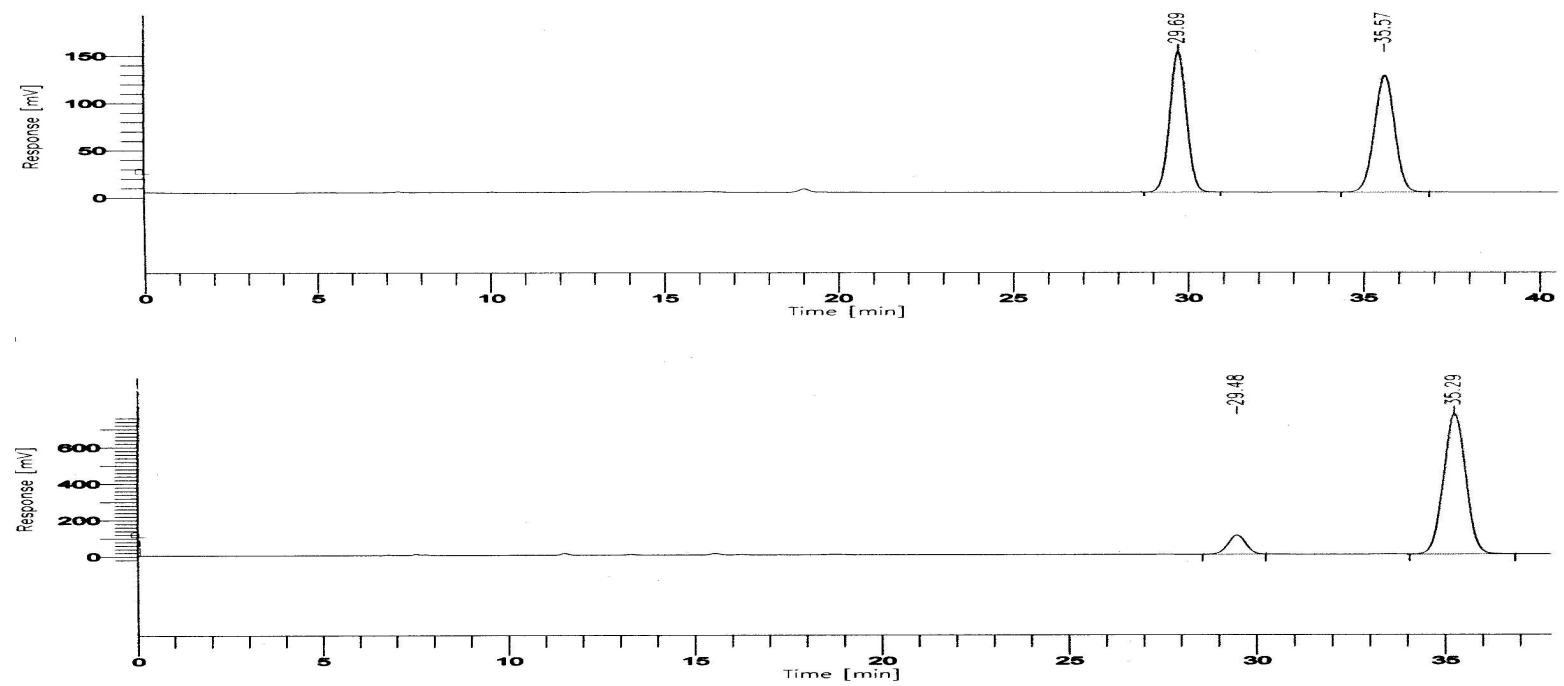

HPLC graph for racemic and enanrioenriched compound (Table 4, Entry 6).

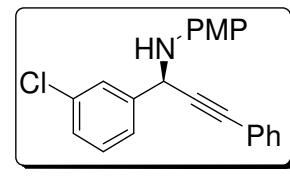

(R)-\{1-(3-Chloro-phenyl)-3-phenyl-prop-2-ynyl\}-(4-methoxy-

phenyl)-amine (Table 4, Entry 7). 82\% ee; isolated in $92 \%$ yield as yellow gel; $\mathrm{R}_{\mathrm{f}}=$ 0.54 (EtOAc: petroleum ether = 1:9); HPLC conditions: Diacel chiralpak AD-H $(4.6 \mathrm{~cm}$ I.D. $\mathrm{x} 25 \mathrm{~cm}$ ), 95:5 hexanes: ${ }^{i}-\mathrm{PrOH}, 1.0 \mathrm{~mL} / \mathrm{min}$ flow rate, $\lambda=254 \mathrm{~nm}, \mathrm{~T}_{\mathrm{R}}$ (major) $=$ 17.13 min and $\mathrm{T}_{\mathrm{R}}$ (minor) = $14.08 \mathrm{~min}$; IR (thin film): 3366 (br), 3056 (br), 2925 (br), 2833 (br), 1593, 1511, 1466, 1420, 1237, 1179, 1072, 1034, 820, 790, 755, $687 \mathrm{~cm}-1$; ${ }^{1} \mathrm{H}$ NMR $\left(\mathrm{CDCl}_{3}, 400 \mathrm{MHz}\right): \delta 7.65(\mathrm{~s}, 1 \mathrm{H}), 7.52-7.53(\mathrm{~m}, 1 \mathrm{H}), 7.38-7.41$ (m, 2H), 7.22$7.32(\mathrm{~m}, 5 \mathrm{H}), 6.71-6.80(\mathrm{~m}, 4 \mathrm{H}), 5.37(\mathrm{~s}, 1 \mathrm{H}), 3.74(\mathrm{~s}, 3 \mathrm{H}) ;{ }^{13} \mathrm{C}\left(\mathrm{CDCl}_{3}, 100 \mathrm{MHz}\right): \delta$ $153.1,141.9,140.2,134.5,131.7,129.9,128.3,128.2,127.5,127.4,125.4,122.5,115.9$, 114.6, 87.9, 85.5, 55.6, 51.3; Anal. cacld. for $\mathrm{C}_{22} \mathrm{H}_{18} \mathrm{ClNO}$ : C, 75.97; H, 5.22; N, 4.03.

Found: C, 76.21; H, 5.29; N, 4.01. Optical Rotation: $[\alpha]^{25}+105.7$ (c 1.8, $\mathrm{CHCl}_{3}$ ). 

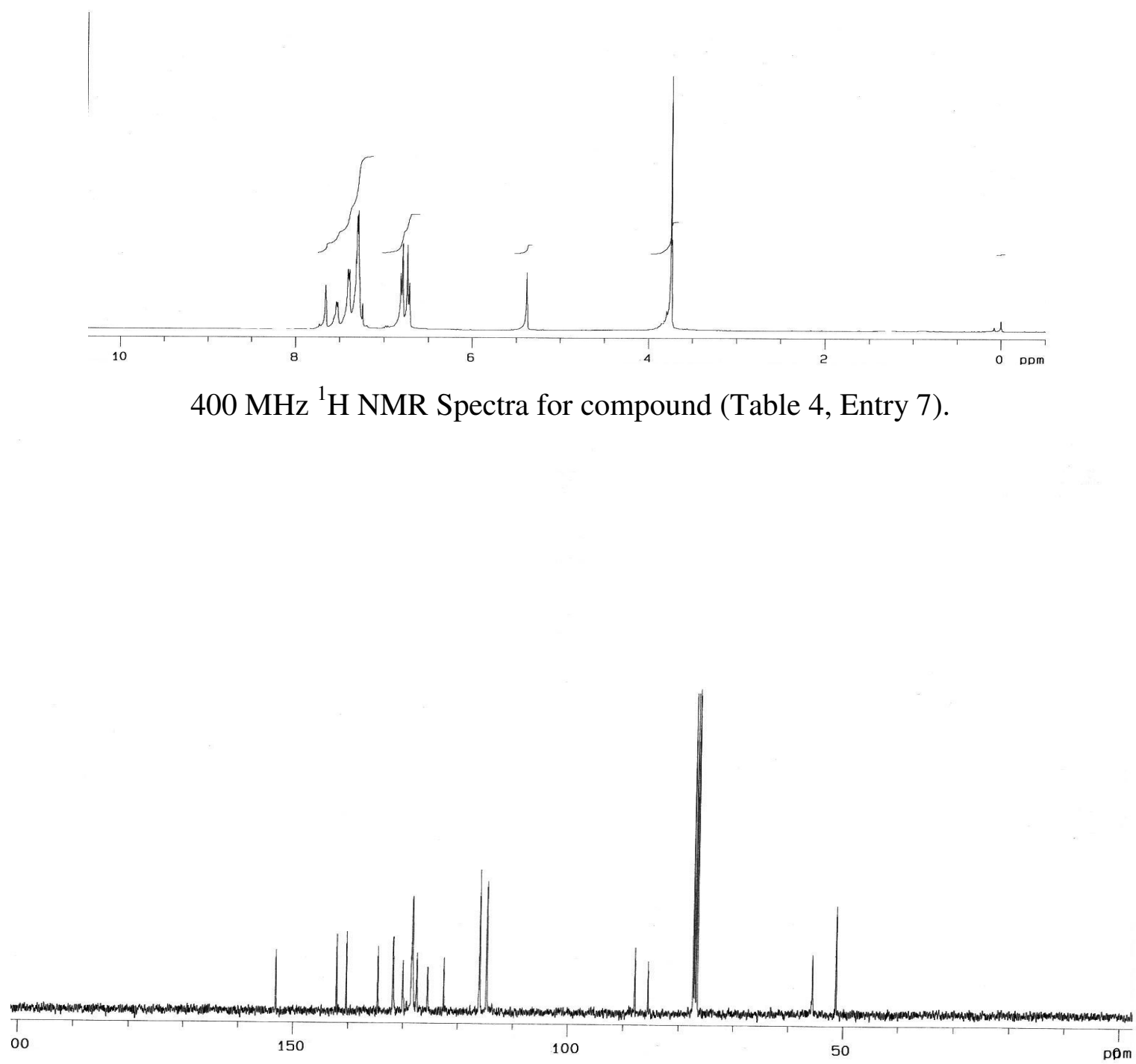

$100 \mathrm{MHz}{ }^{13} \mathrm{C}$ NMR Spectra for compound (Table 4, Entry 7). 

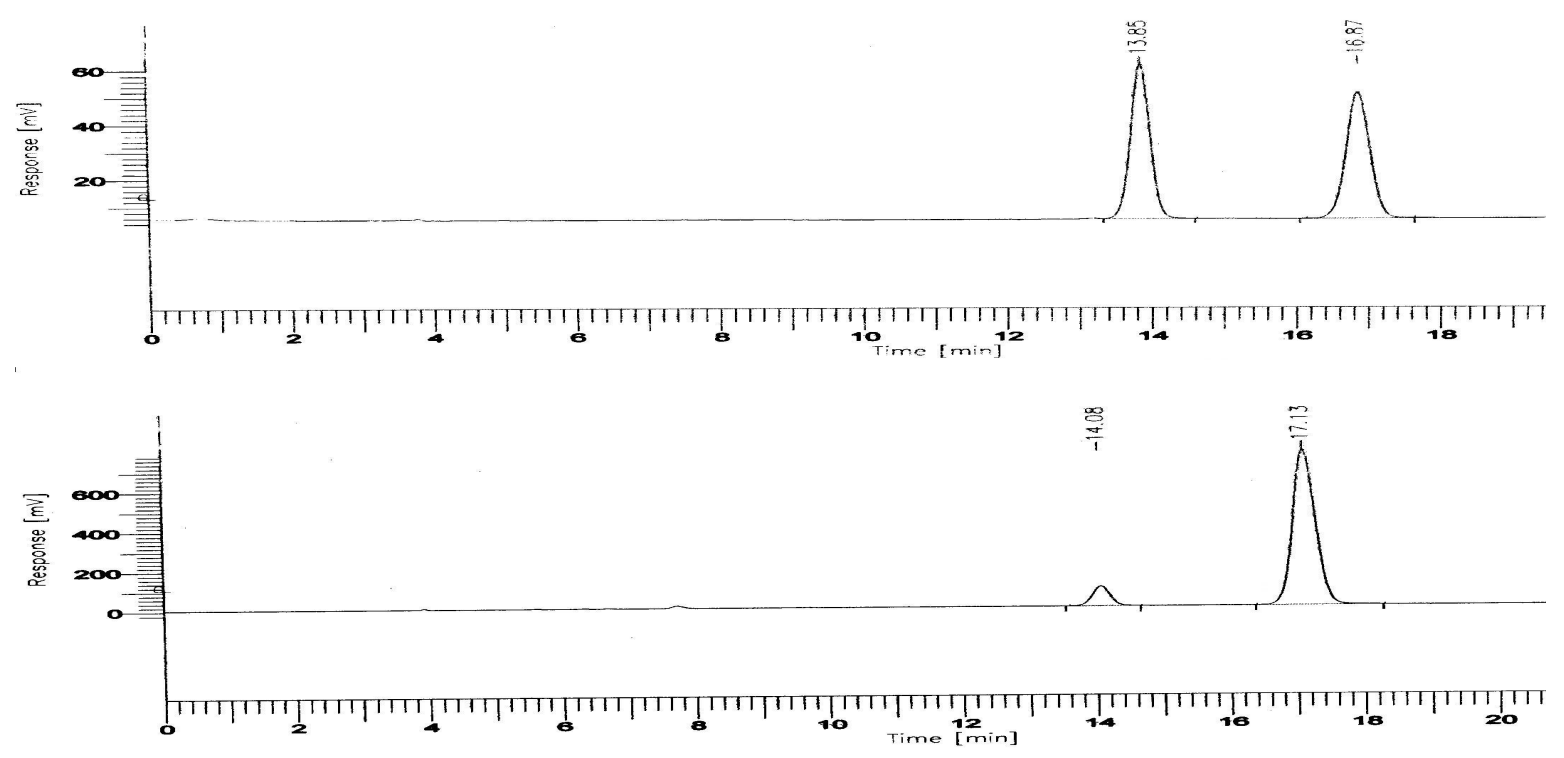

HPLC graph for racemic and enanrioenriched compound (Table 4, Entry 7).

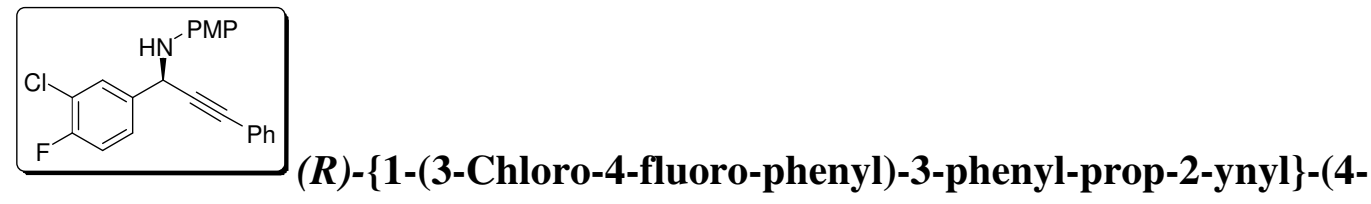

methoxy-phenyl)-amine (Table 4, Entry 8). 86\% ee; isolated in $90 \%$ yield as yellow gel; $\mathrm{R}_{\mathrm{f}}=0.48$ (EtOAc: petroleum ether $\left.=1: 9\right)$; HPLC conditions: Diacel chiralpak AD-H (4.6 cm I.D. x $25 \mathrm{~cm}$ ), 95:5 hexanes: ${ }^{i}-\mathrm{PrOH}, 1.0 \mathrm{~mL} / \mathrm{min}$ flow rate, $\lambda=254 \mathrm{~nm}, \mathrm{~T}_{\mathrm{R}}$ $($ major $)=20.51 \mathrm{~min}$ and $\mathrm{T}_{\mathrm{R}}($ minor $)=17.19 \mathrm{~min} ;{ }^{1} \mathrm{H} \mathrm{NMR}\left(\mathrm{CDCl}_{3}, 400 \mathrm{MHz}\right): \delta 7.71$ $(\mathrm{dd}, J=6.84,2.20 \mathrm{~Hz}, 1 \mathrm{H}), 7.49-7.53(\mathrm{~m}, 1 \mathrm{H}), 7.38-7.41(\mathrm{~m}, 2 \mathrm{H}), 7.26-7.32(\mathrm{~m}, 3 \mathrm{H})$, $7.14(\mathrm{t}, J=8.56 \mathrm{~Hz}, 1 \mathrm{H}), 6.75(\mathrm{ddt}, J=35.88,9.04,2.44 \mathrm{~Hz}, 4 \mathrm{H}), 5.36(\mathrm{~s}, 1 \mathrm{H}), 3.74$ (s, $3 \mathrm{H}) ;{ }^{13} \mathrm{C}\left(\mathrm{CDCl}_{3}, 100 \mathrm{MHz}\right): \delta 158.9,156.4,153.2,140.0,137.0,131.7,129.5,128.5$, 128.3, 127.0, 126.9, 122.3, 116.7, 116.5, 116.0, 114.7, 87.7, 85.7, 55.6, 50.8; Anal. cacld. for $\mathrm{C}_{22} \mathrm{H}_{17} \mathrm{ClFNO}$ : C, 72.23; H, 4.68; N, 3.83. Found: C, 72.49; H, 4.71; N, 3.88. Optical Rotation: $[\alpha]^{25}+89.2\left(\mathrm{c} 0.8, \mathrm{CHCl}_{3}\right)$. 

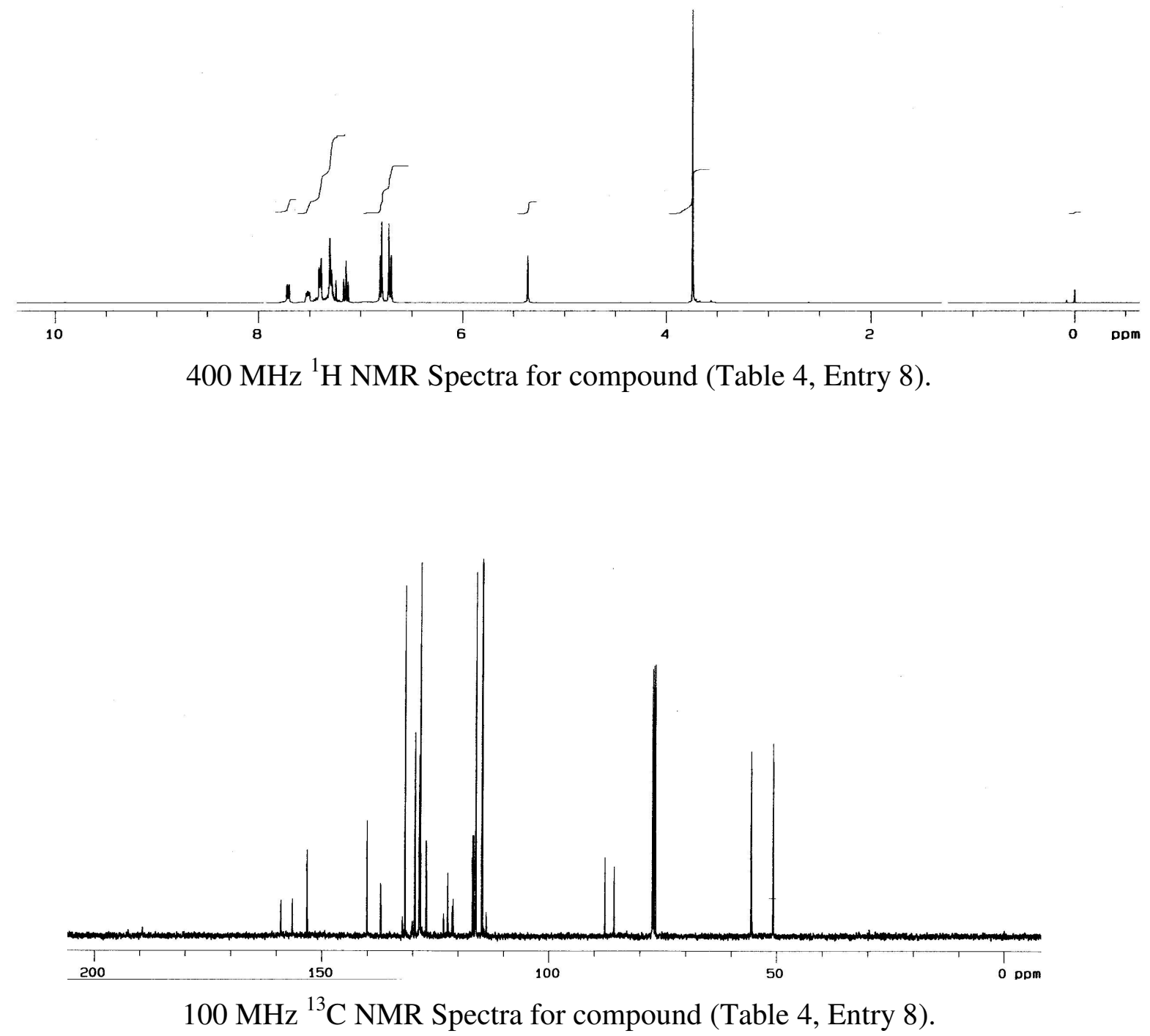

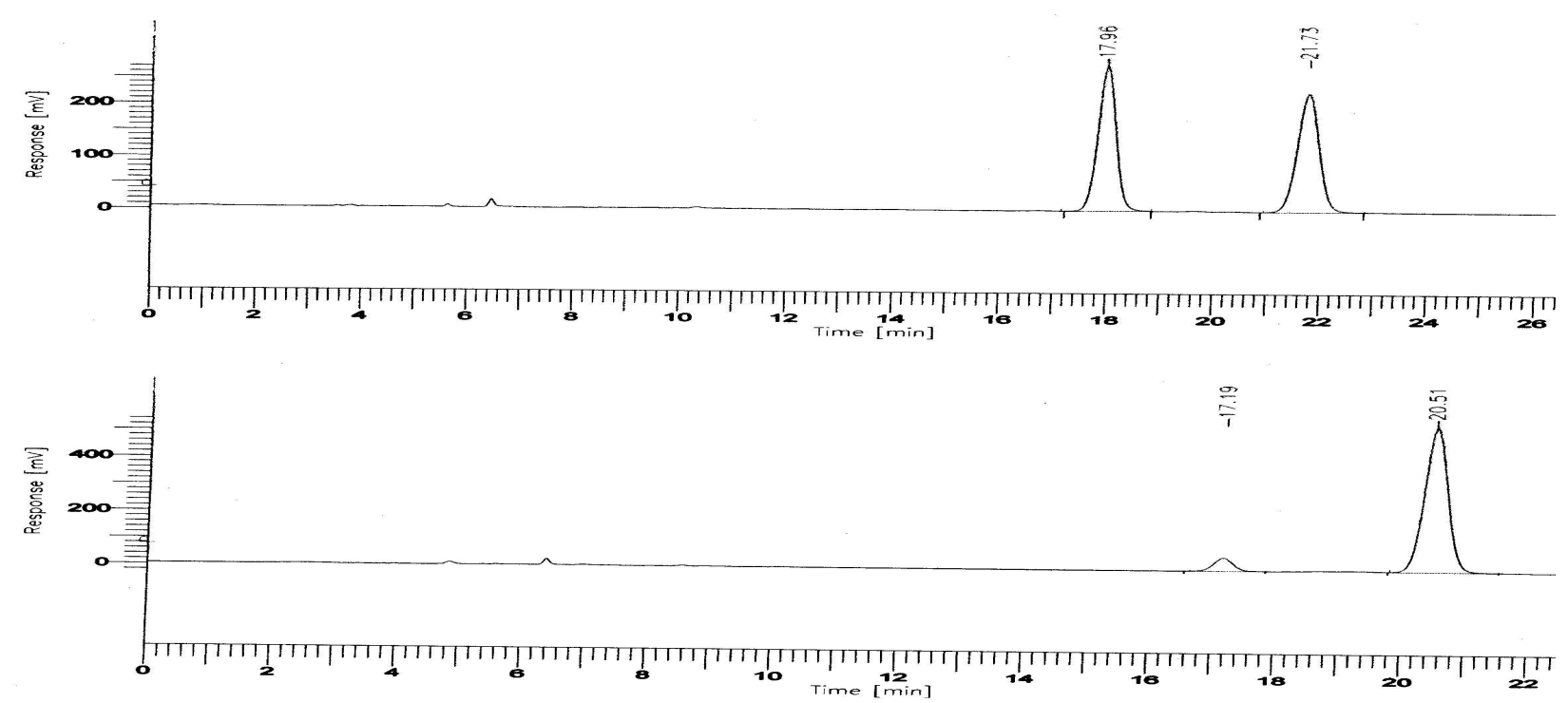

HPLC graph for racemic and enanrioenriched compound (Table 4, Entry 8).

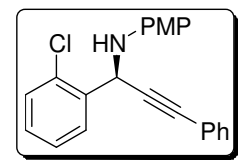

(S)-\{1-(2-Chloro-phenyl)-3-phenyl-prop-2-ynyl\}-(4-methoxy-phenyl)-

amine (Table 4, Entry 9). 97\% ee; isolated in 94\% yield as yellow gel; $\mathrm{R}_{\mathrm{f}}=0.54$

(EtOAc: petroleum ether = 1:9); HPLC conditions: Diacel chiralpak AD-H (4.6 cm I.D. $\mathrm{x}$ $25 \mathrm{~cm}$ ), 95:5 hexanes: ${ }^{i}-\mathrm{PrOH}, 1.0 \mathrm{~mL} / \mathrm{min}$ flow rate, $\lambda=254 \mathrm{~nm}, \mathrm{~T}_{\mathrm{R}}$ (major) $=19.96 \mathrm{~min}$ and $\mathrm{T}_{\mathrm{R}}($ minor $)=13.65 \mathrm{~min}$; IR (thin film): 3398 (br), 3060, 2932, 2832, 1595, 1511, 1467, 1441, 1407, 1238, 1180, 1124, 1036, 820, 755, $692 \mathrm{~cm}-1 ;{ }^{1} \mathrm{H}$ NMR $\left(\mathrm{CDCl}_{3}, 400\right.$ MHz): $\delta 7.75(\mathrm{dd}, J=7.56,1.72 \mathrm{~Hz}, 1 \mathrm{H}), 7.32-7.41(\mathrm{~m}, 3 \mathrm{H}), 7.16-7.27$ (m, 5H), 6.65$6.78(\mathrm{~m}, 4 \mathrm{H}), 5.66(\mathrm{~s}, 1 \mathrm{H}), 3.67(\mathrm{~s}, 3 \mathrm{H}) ;{ }^{13} \mathrm{C}\left(\mathrm{CDCl}_{3}, 100 \mathrm{MHz}\right): \delta 153.0,140.3,137.2$, 133.2, 131.7, 129.8, 129.2, 128.7, 128.3, 128.1, 127.2, 122.5, 115.9, 114.6, 87.8, 84.9, 55.5, 49.3; Anal. cacld. for $\mathrm{C}_{22} \mathrm{H}_{18}$ ClNO: C, 75.97; H, 5.22; N, 4.03. Found: C, 76.19; H, 5.31; N, 4.09. Optical Rotation: $[\alpha]^{25}+100.4$ (c 2.2, $\left.\mathrm{CHCl}_{3}\right)$. 


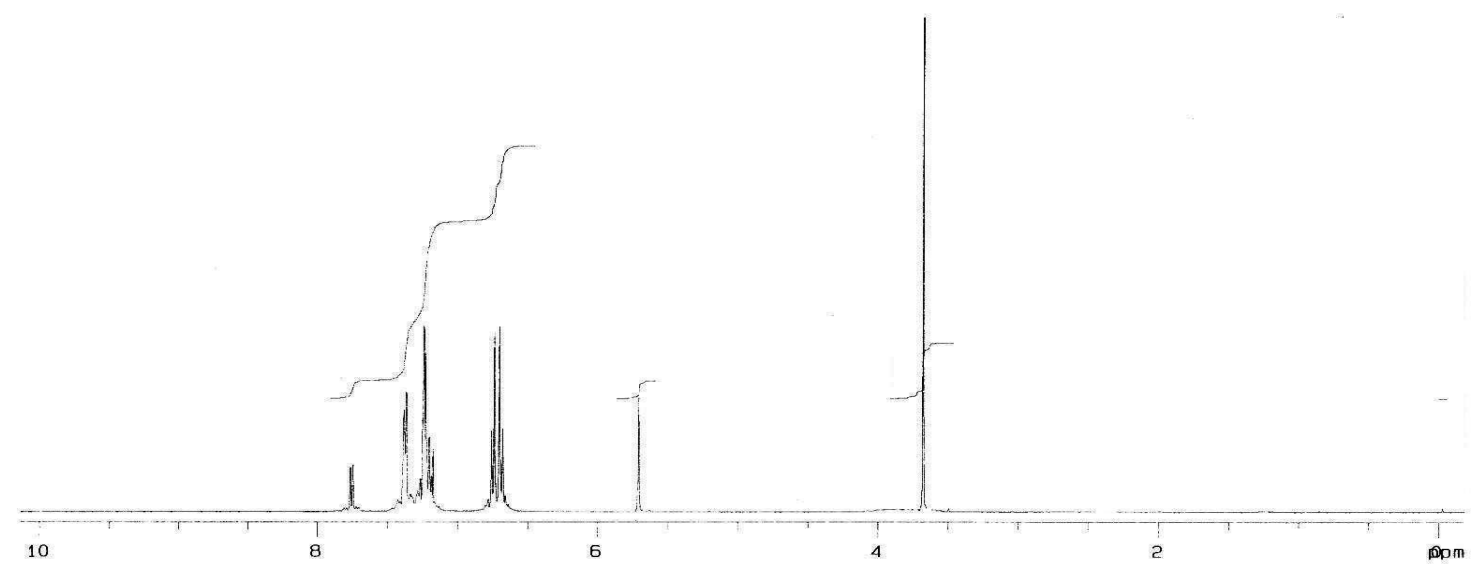

$400 \mathrm{MHz}{ }^{1} \mathrm{H}$ NMR Spectra for compound (Table 4, Entry 9).

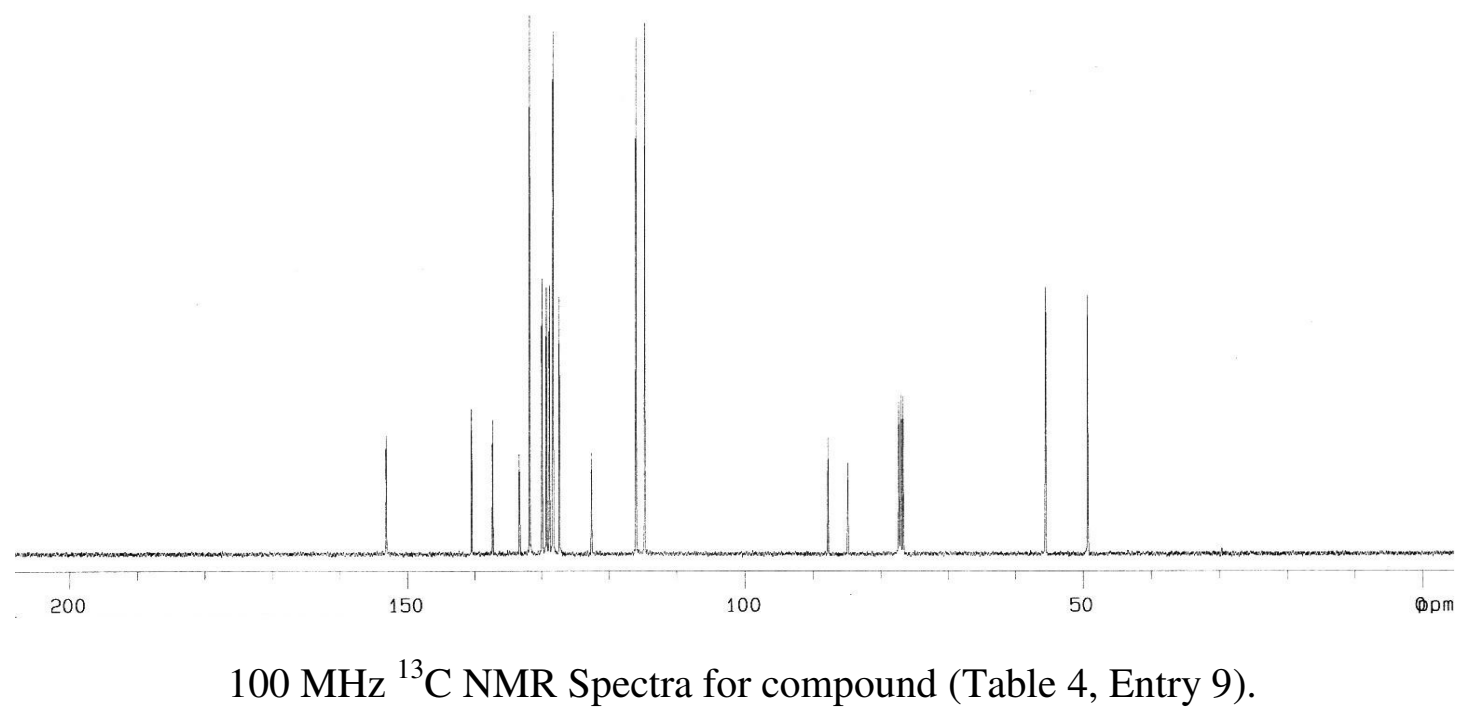



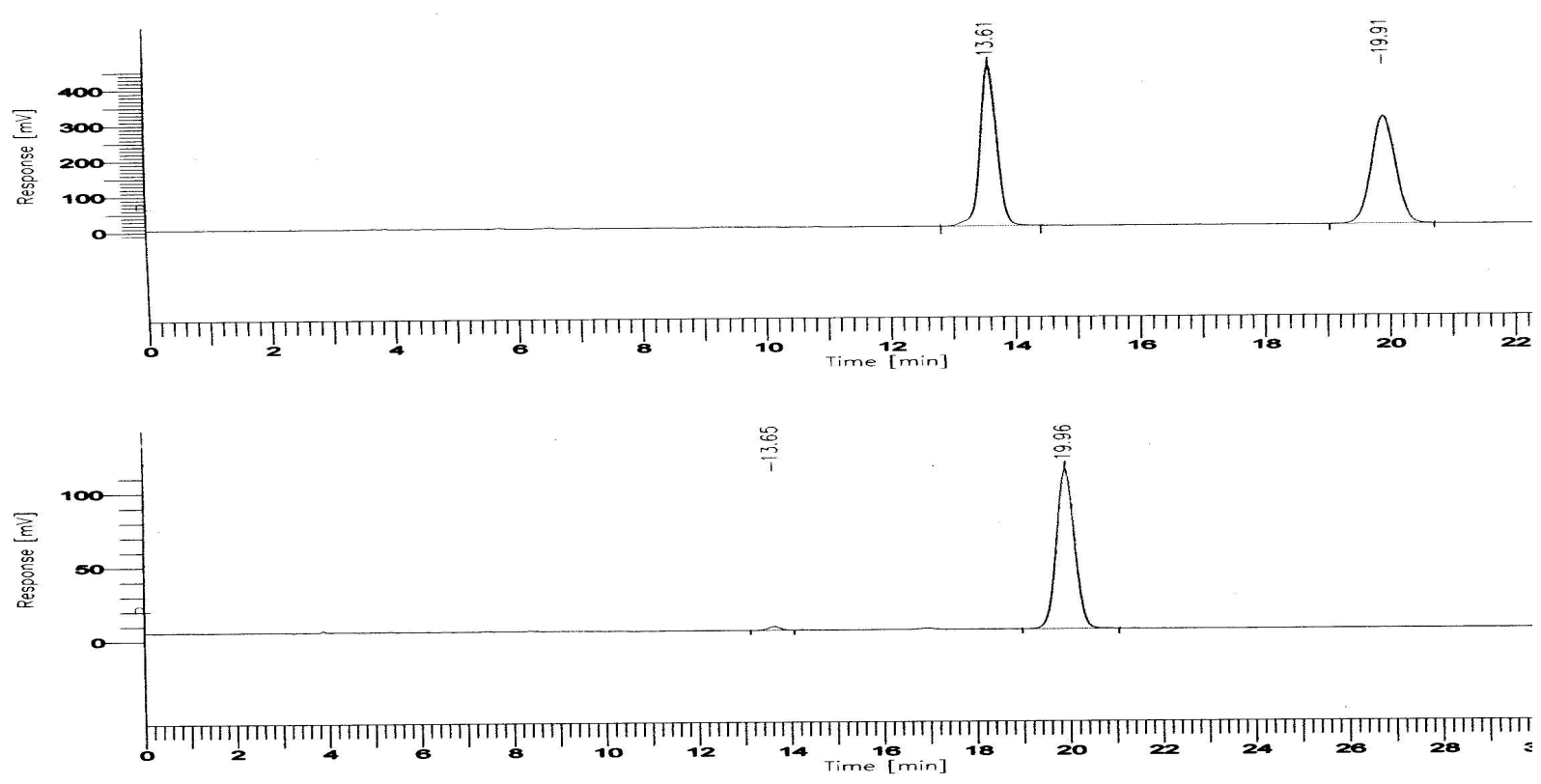

HPLC graph for racemic and enanrioenriched compound (Table 4, Entry 9).

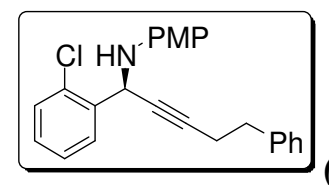

(S)-\{1-(2-chloro-phenyl)-5-phenyl-pent-2-ynyl)-(4-methoxy-

phenyl)-amine (Table 4, Entry 10). $85 \%$ ee; isolated in $61 \%$ yield as yellow gel; $R_{\mathrm{f}}=$ 0.54 (EtOAc: petroleum ether = 1:9); HPLC conditions: Diacel chiralpak AD-H $(4.6 \mathrm{~cm}$ I.D. $x 25 \mathrm{~cm}$ ), 95:5 hexanes: ${ }^{i}-\mathrm{PrOH}, 1.0 \mathrm{~mL} / \mathrm{min}$ flow rate, $\lambda=254 \mathrm{~nm}, \mathrm{~T}_{\mathrm{R}}$ (major) $=$ $21.15 \mathrm{~min}$ and $\mathrm{T}_{\mathrm{R}}$ (minor) = $12.96 \mathrm{~min}$; IR (thin film): 3392 (br), 3028, 2927, 2856, 1597 , 1511, 1445, 1283, 1179, 1119. 1036, 820, 743, $\left.699 \mathrm{~cm}-1 ;{ }^{1} \mathrm{H} \mathrm{NMR} \mathrm{(CDCl} 3,400 \mathrm{MHz}\right): \delta$ 7.59-7.61 (m, 1H), 7.36-7.38 (m. 1H), 7.14-7.27 (m, 7H), $6.70(\mathrm{dd}, J=53.6,8.80 \mathrm{~Hz}$, 2H), $5.45(\mathrm{~s}, 1 \mathrm{H}), 3.72(\mathrm{~s}, 3 \mathrm{H}), 2.79(\mathrm{t}, J=7.32 \mathrm{~Hz}, 2 \mathrm{H}), 2.51(\mathrm{t}, J=7.56 \mathrm{~Hz}, 2 \mathrm{H}) ;{ }^{13} \mathrm{C}$ $\left(\mathrm{CDCl}_{3}, 100 \mathrm{MHz}\right): \delta 152.9,140.5,137.7,133.1,129.7,129.0,128.6,128.5,128.3$, 127.1, 126.2, 115.6, 114.6, 84.6, 79.6, 55.6, 48.7, 34.8, 20.9; Anal. cacld. for $\mathrm{C}_{24} \mathrm{H}_{22} \mathrm{ClNO}$ : C, 76.69; H, 5.90; N, 3.73. Found: C, 76.93; H, 5.96; N, 3.75. Optical Rotation: $[\alpha]^{25}+51.4\left(\mathrm{c} 1.4, \mathrm{CHCl}_{3}\right)$. 

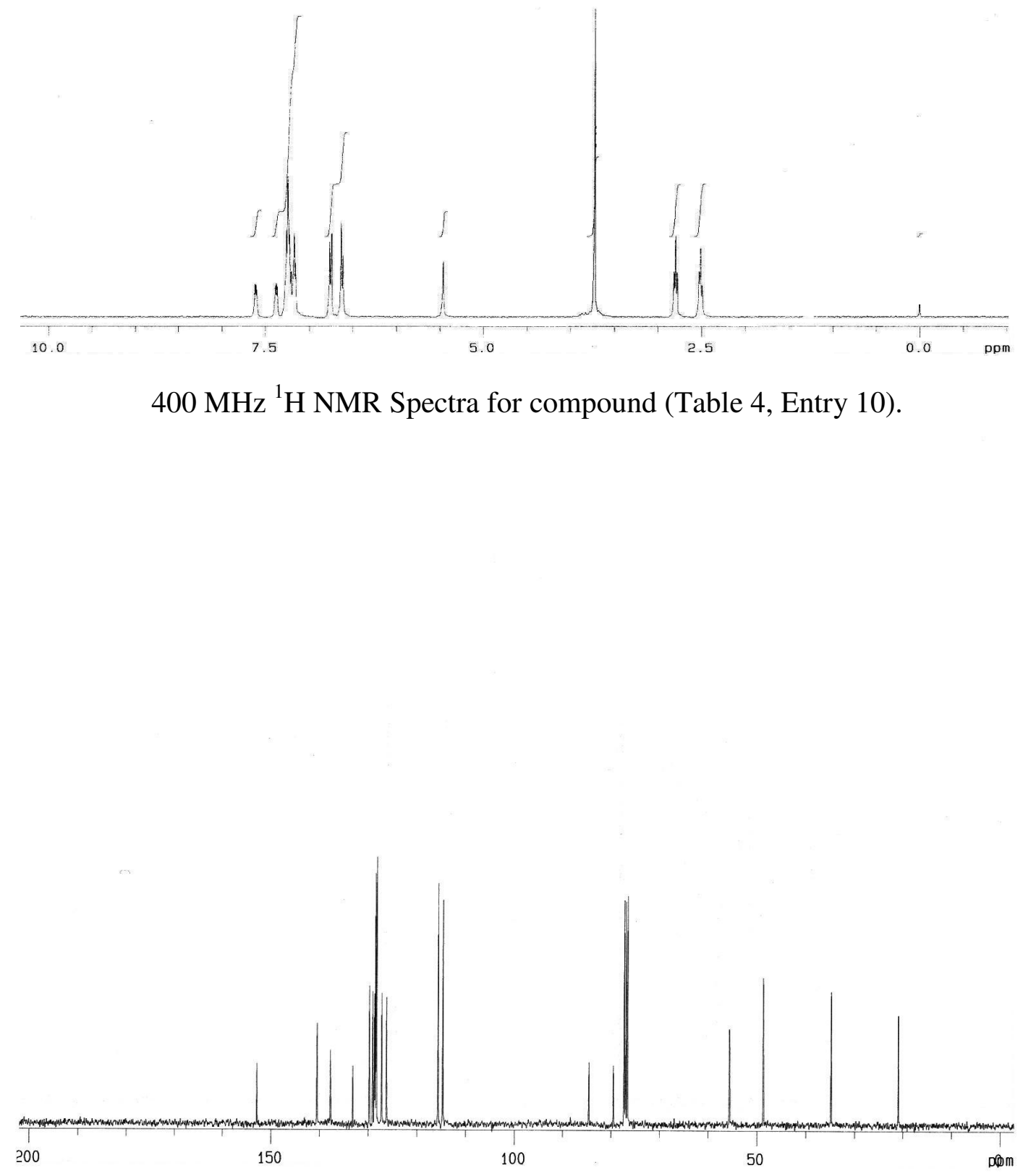

$100 \mathrm{MHz}{ }^{13} \mathrm{C}$ NMR Spectra for compound (Table 4, Entry 10). 

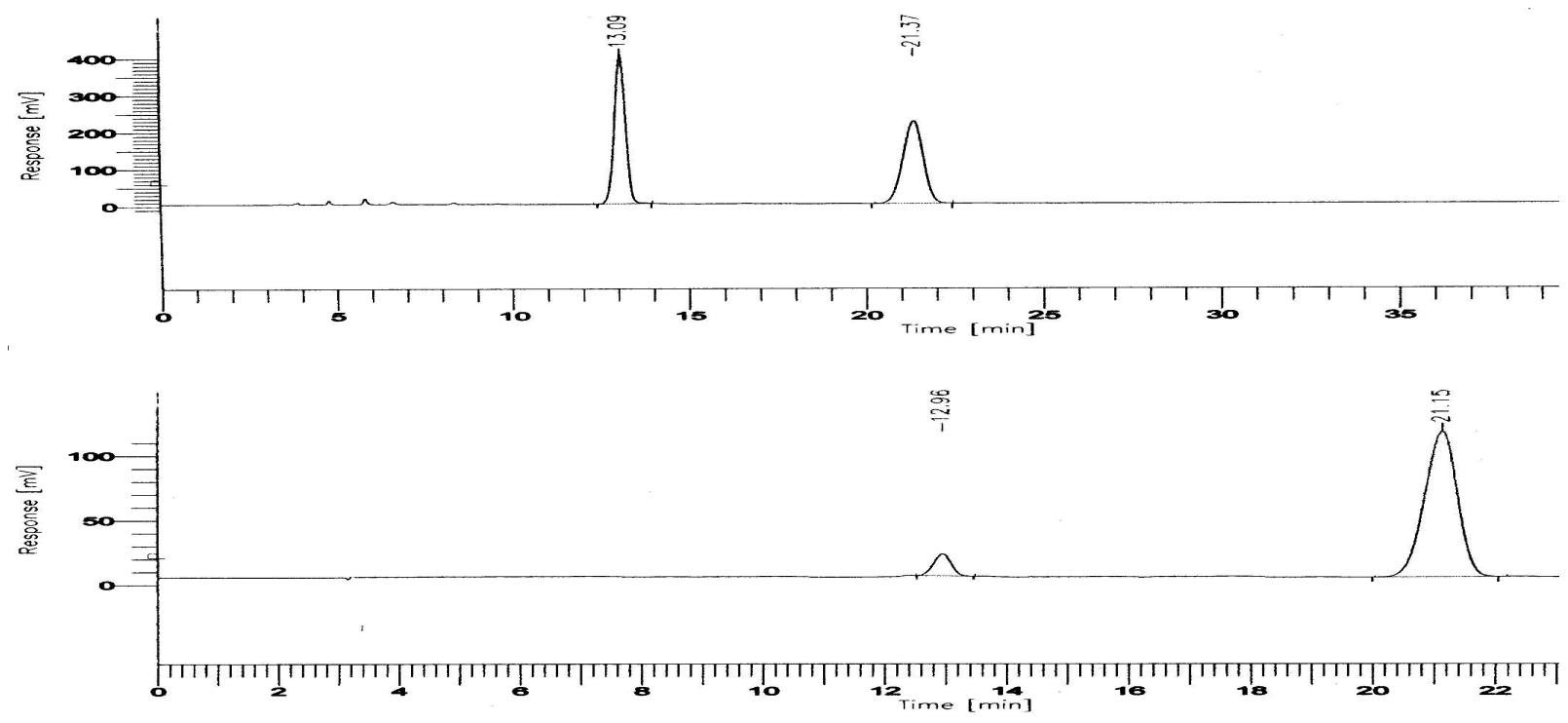

HPLC graph for racemic and enanrioenriched compound (Table 4, Entry 10).

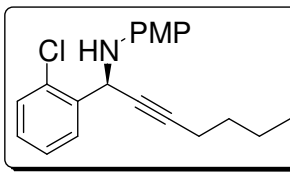

(S)-\{1-(2-Chloro-phenyl-hept-2-ynyl)\}-(4-methoxy-phenyl)-amine

(Table 4, Entry 11). 87\% ee; isolated in 67\% yield as yellow gel; $R_{f}=0.60$ (EtOAc:

petroleum ether = 1:9); HPLC conditions: Diacel chiralpak AD-H $(4.6 \mathrm{~cm}$ I.D. x $25 \mathrm{~cm})$, 95:5 hexanes: ${ }^{i}-\mathrm{PrOH}, 0.5 \mathrm{~mL} / \mathrm{min}$ flow rate, $\lambda=254 \mathrm{~nm}, \mathrm{~T}_{\mathrm{R}}$ (major) $=11.23 \mathrm{~min}$ and $\mathrm{T}_{\mathrm{R}}$ (minor) $=8.00 \mathrm{~min}$; IR (thin film): 3385, 3061, 2956, 2931, 2865, 1593, 1512, 1466, 1239, 1120, 1038, 820, $756 \mathrm{~cm}-1 ;{ }^{1} \mathrm{H} \mathrm{NMR}\left(\mathrm{CDCl}_{3}, 400 \mathrm{MHz}\right): \delta 7.72$ (dd, $J=7.56,1.96$ $\mathrm{Hz}, 1 \mathrm{H}), 7.37$ (dd, $J=7.44,1.40 \mathrm{~Hz}, 1 \mathrm{H}), 7.19-7.28$ (m, 2H), 6.70 (dd, $J=37.72,12.44$ $\mathrm{Hz}, 4 \mathrm{H}), 5.48(\mathrm{~s}, 1 \mathrm{H}), 3.71$ (s, 3H), 2.18-2.22 (m, 2H), 1.43-1.48 (m, 2H), 1.25-1.38 (m, $2 \mathrm{H}), 0.87(\mathrm{t}, J=7.32 \mathrm{~Hz}, 3 \mathrm{H}) ;{ }^{13} \mathrm{C}\left(\mathrm{CDCl}_{3}, 100 \mathrm{MHz}\right): \delta 152.8,140.5,137.9,133.1$, 129.7, 128.9, 128.6, 127.1, 115.7, 114.5, 85.6, 78.6, 55.5, 48.8, 30.6, 21.8, 18.4, 13.5; Anal. cacld. for $\mathrm{C}_{20} \mathrm{H}_{22}$ ClNO: C, 73.27; H, 6.76; N, 4.27. Found: C, 73.51; H, 6.82; N, 4.29. Optical Rotation: $[\alpha]_{\mathrm{D}}^{25}+64.8\left(\mathrm{c} 1.2, \mathrm{CHCl}_{3}\right)$. 


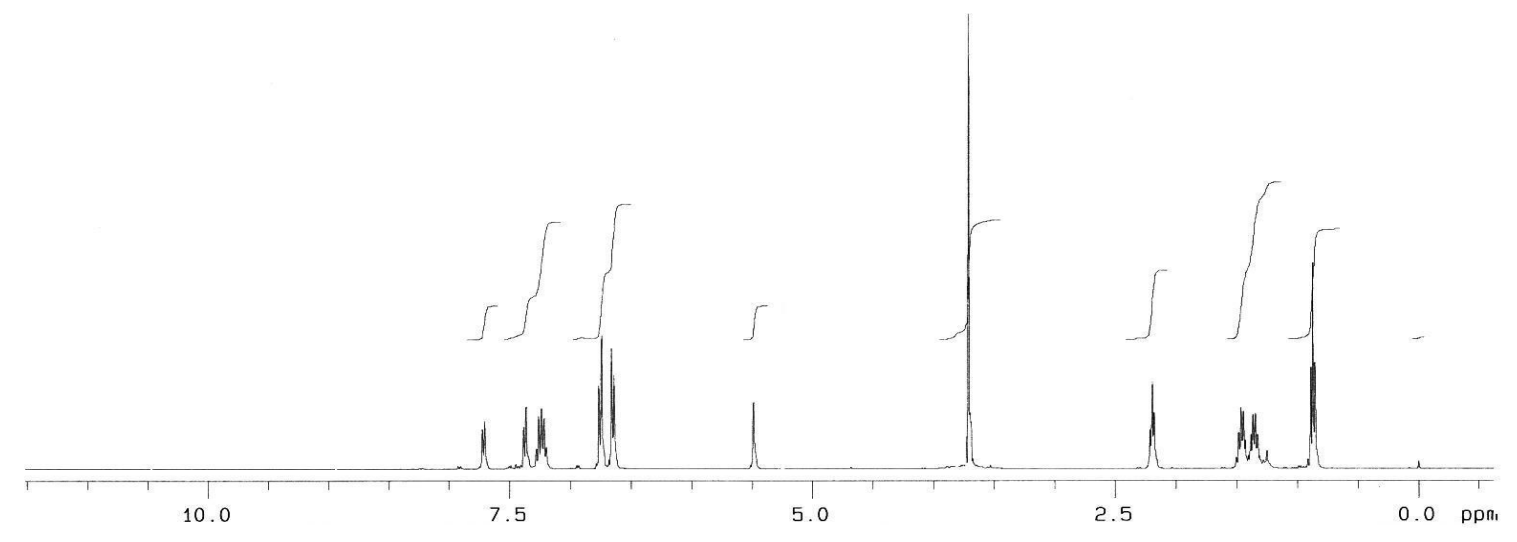

$400 \mathrm{MHz}{ }^{1} \mathrm{H}$ NMR Spectra for compound (Table 4, Entry 11).

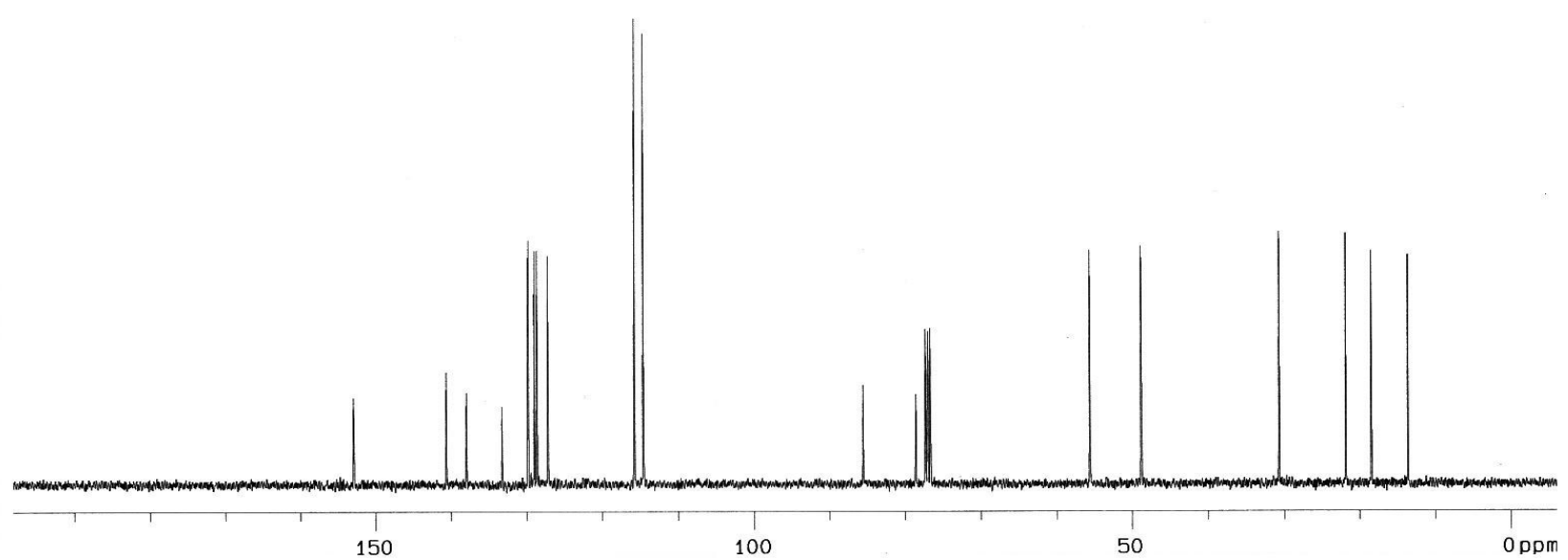

$100 \mathrm{MHz}{ }^{13} \mathrm{C}$ NMR Spectra for compound (Table 4, Entry 11). 

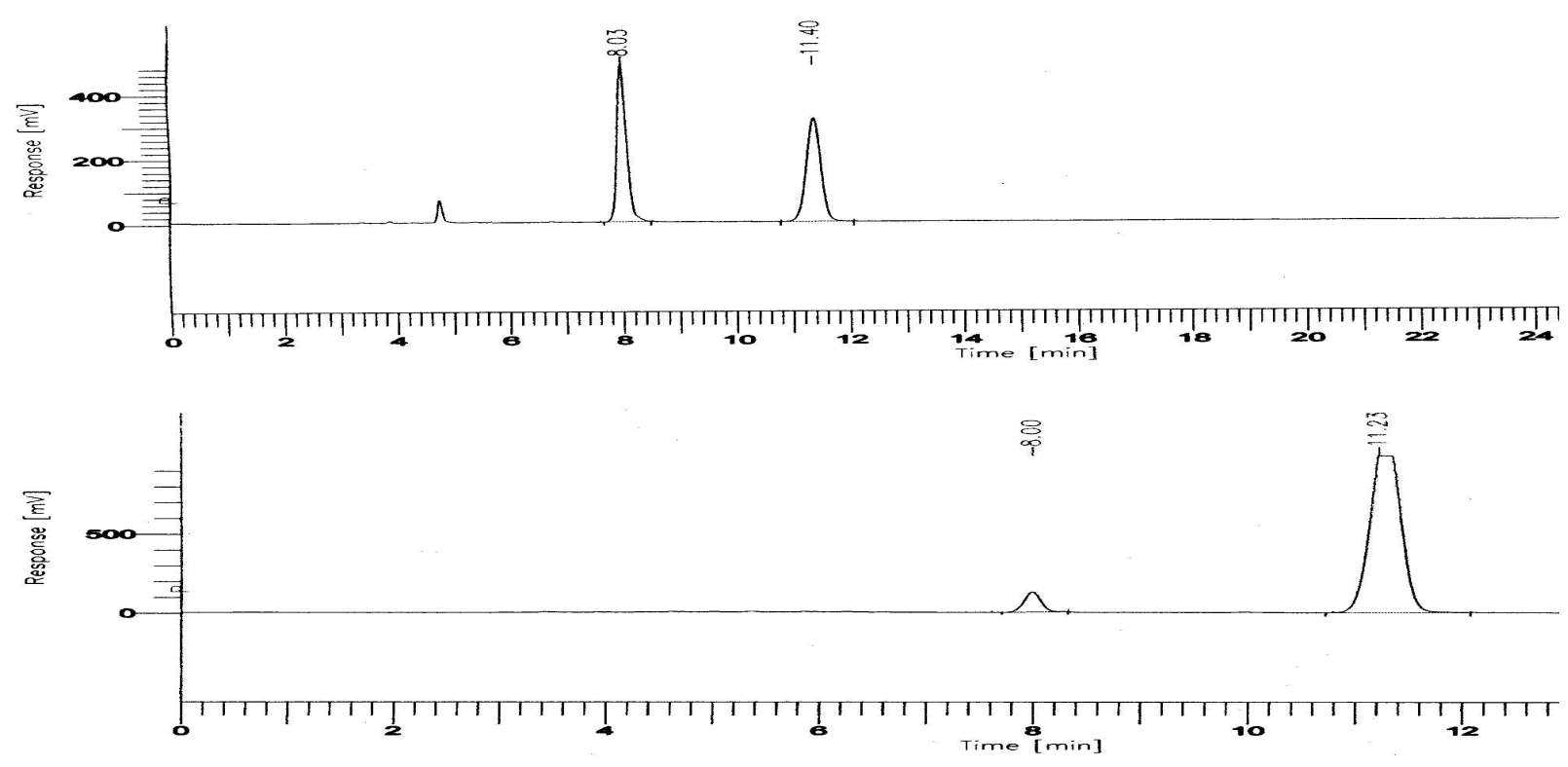

HPLC graph for racemic and enanrioenriched compound (Table 4, Entry 11).

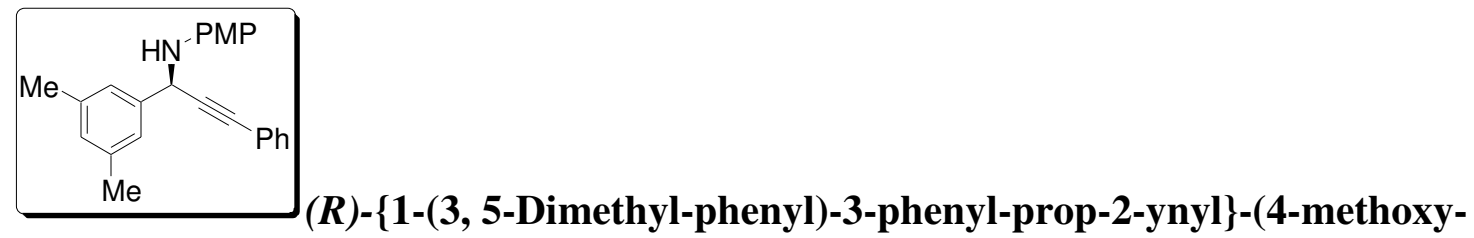

phenyl)-amine (Table 4, Entry 12). 93\% ee; isolated in $98 \%$ yield as yellow gel; $R_{\mathrm{f}}=$ 0.58 (EtOAc: petroleum ether = 1:9); HPLC conditions: Diacel chiralpak AD-H $(4.6 \mathrm{~cm}$ I.D. $\mathrm{x} 25 \mathrm{~cm}$ ), 95:5 hexanes: ${ }^{i}-\mathrm{PrOH}, 1.0 \mathrm{~mL} / \mathrm{min}$ flow rate, $\lambda=254 \mathrm{~nm}, \mathrm{~T}_{\mathrm{R}}$ (major) $=$ $11.08 \mathrm{~min}$ and $\mathrm{T}_{\mathrm{R}}($ minor $)=10.35 \mathrm{~min}$; IR (thin film): 3368 (br), 2927, 2857, 1738, 1602, $1511,1461,1241,1172,1034,821,738,698 \mathrm{~cm}-1 ;{ }^{1} \mathrm{H} \mathrm{NMR}\left(\mathrm{CDCl}_{3}, 400 \mathrm{MHz}\right): \delta 7.24-$ $7.32(\mathrm{~m}, 2 \mathrm{H}), 7.13-7.19(\mathrm{~m}, 5 \mathrm{H}), 6.86(\mathrm{~s}, 1 \mathrm{H}), 6.66-6.73(\mathrm{~m}, 4 \mathrm{H}), 5.23(\mathrm{~s}, 1 \mathrm{H}), 3.65$ (s, $3 \mathrm{H}), 2.25(\mathrm{~s}, 6 \mathrm{H}) ;{ }^{13} \mathrm{C}\left(\mathrm{CDCl}_{3}, 100 \mathrm{MHz}\right): \delta 152.8,140.8,139.8,138.3,131.7,129.7$, 128.1, 125.7, 125.1, 122.9, 115.8, 114.6, 89.0, 84.8, 55.6, 51.7, 21.3; Anal. cacld. for $\mathrm{C}_{24} \mathrm{H}_{23} \mathrm{NO}$ : C, 84.42; H, 6.79; N, 4.10. Found: C, 84.69; H, 6.83; N, 4.16. Optical Rotation: $[\alpha]^{25}+107.8\left(\mathrm{c} 0.8, \mathrm{CHCl}_{3}\right)$. 


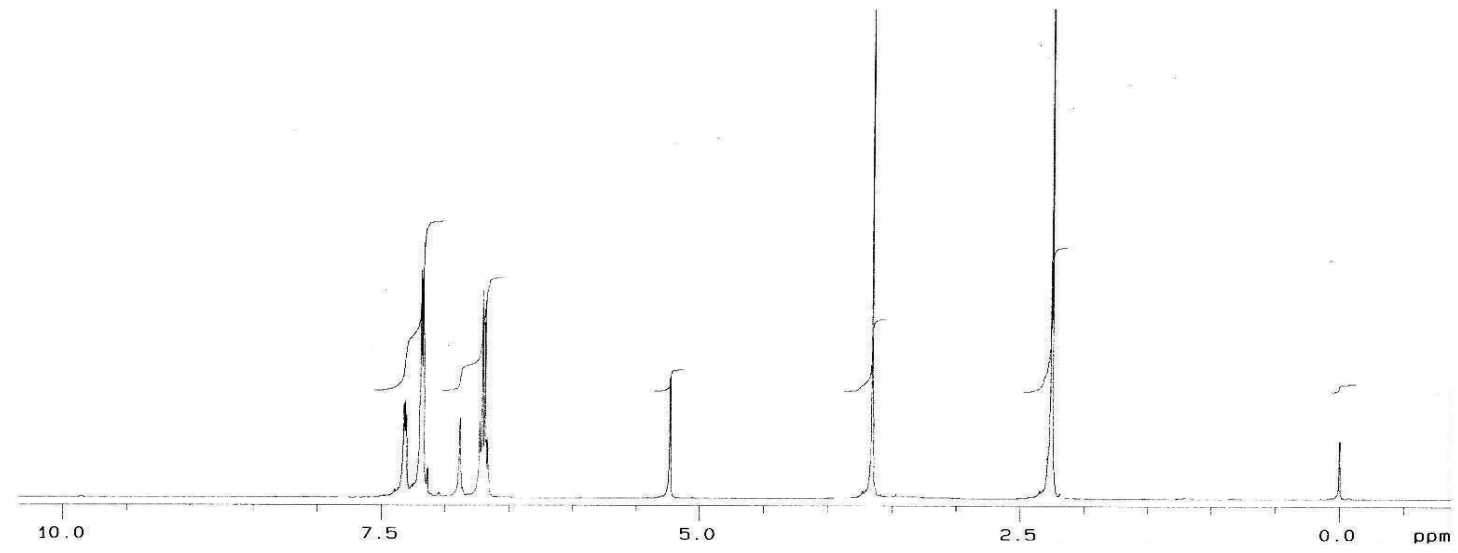

$400 \mathrm{MHz}{ }^{1} \mathrm{H}$ NMR Spectra for compound (Table 4, Entry 12).

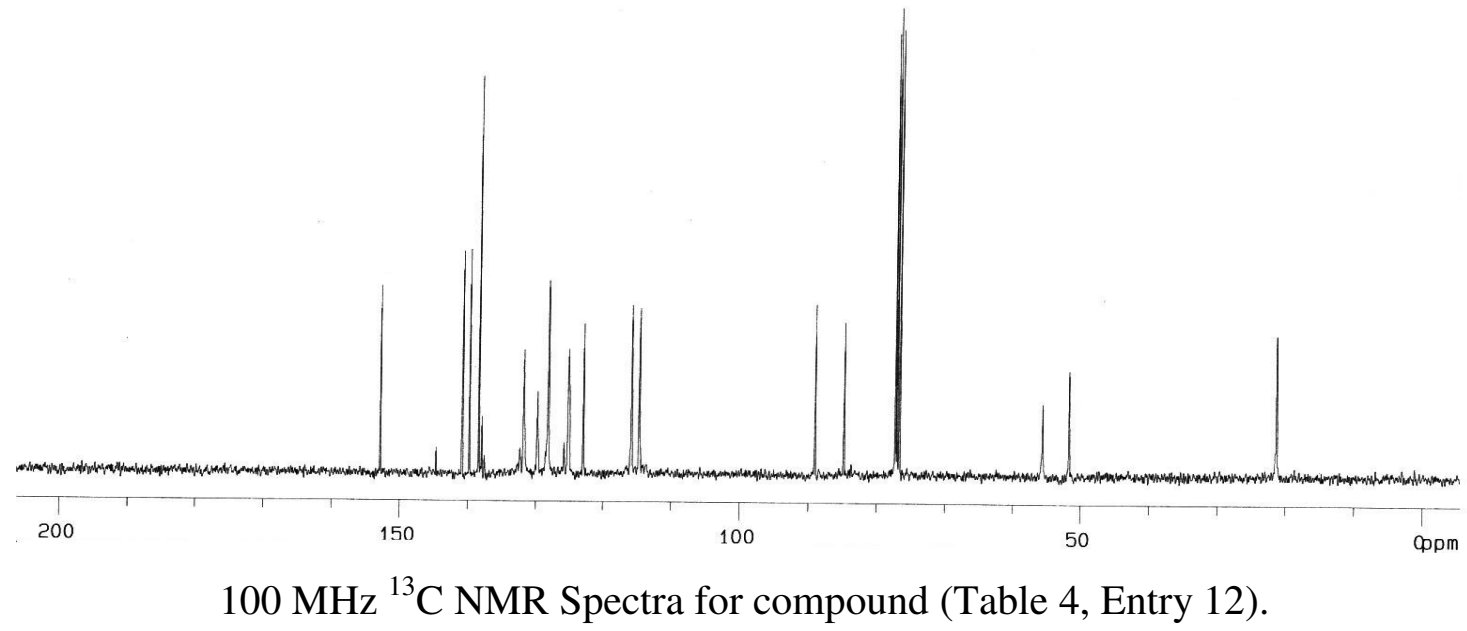



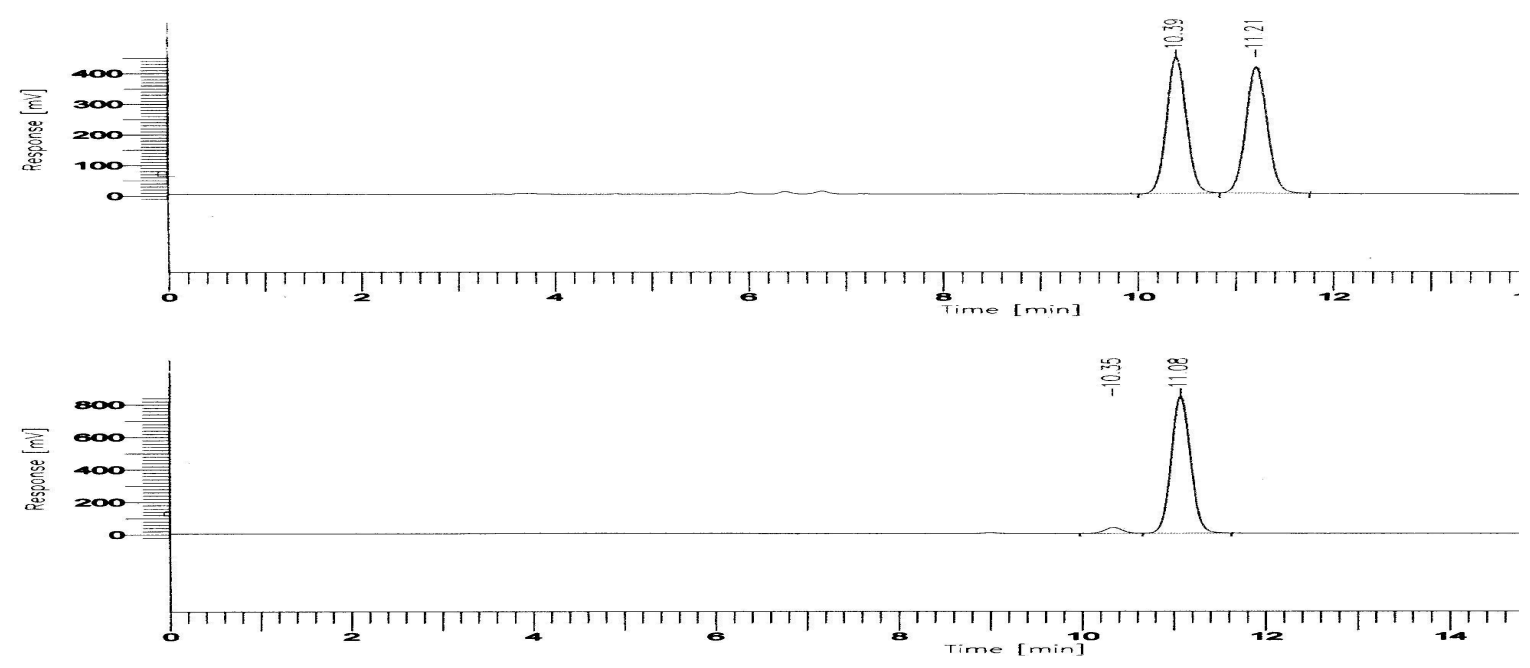

HPLC graph for racemic and enanrioenriched compound (Table 4, Entry 12).

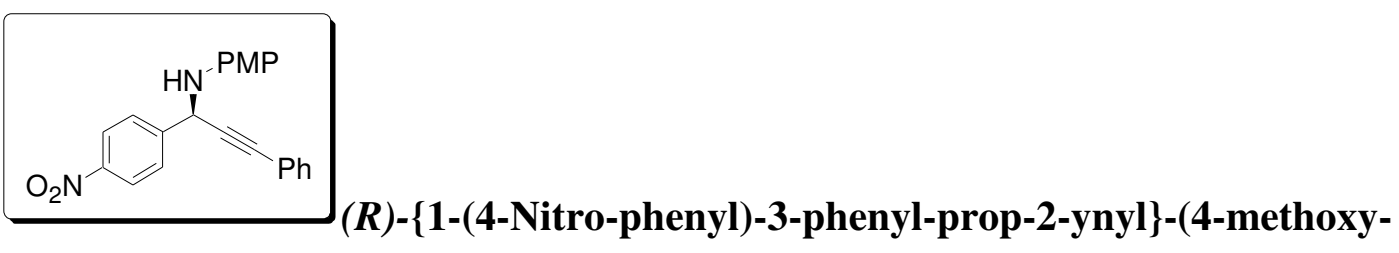

phenyl)-amine (Table 4, Entry 13). $90 \%$ ee; isolated in $90 \%$ yield as yellow gel; $\mathrm{R}_{\mathrm{f}}=$ 0.58 (EtOAc: petroleum ether $=2: 8)$; HPLC conditions: Diacel chiralpak AD-H $(4.6 \mathrm{~cm}$ I.D. $x 25 \mathrm{~cm}$ ), 90:10 hexanes: ${ }^{i}-\mathrm{PrOH}, 1.0 \mathrm{~mL} / \mathrm{min}$ flow rate, $\lambda=254 \mathrm{~nm}, \mathrm{~T}_{\mathrm{R}}$ (major) $=$ $45.61 \mathrm{~min}$ and $\mathrm{T}_{\mathrm{R}}$ (minor) $=37.45 \mathrm{~min} ;{ }^{1} \mathrm{H}$ NMR $\left(\mathrm{CDCl}_{3}, 400 \mathrm{MHz}\right): \delta 8.22(\mathrm{~d}, J=8.52$ $\mathrm{Hz}, 2 \mathrm{H}), 7.81(\mathrm{~d}, J=8.80 \mathrm{~Hz}, 2 \mathrm{H}), 7.33(\mathrm{~m}, 2 \mathrm{H}), 7.30(\mathrm{~m}, 2 \mathrm{H}), 6.95(\mathrm{~m}, 2 \mathrm{H}), 6.79$ (d, $J=$ $9.00 \mathrm{~Hz}, 2 \mathrm{H}), 6.69(\mathrm{~d}, J=9.00 \mathrm{~Hz}, 2 \mathrm{H}), 5.48(\mathrm{~s}, 1 \mathrm{H}), 3.73$ (s, 3H); Anal. cacld. for $\mathrm{C}_{22} \mathrm{H}_{18} \mathrm{~N}_{2} \mathrm{O}_{3}$ : C, 73.73; H, 5.06; N, 7.82. Found: C, 73.56; H, 5.11; N, 7.94. Optical Rotation: $[\alpha]^{25}{ }_{\mathrm{D}}+49.5$ (c 1.0, $\mathrm{CHCl}_{3}$ ). 


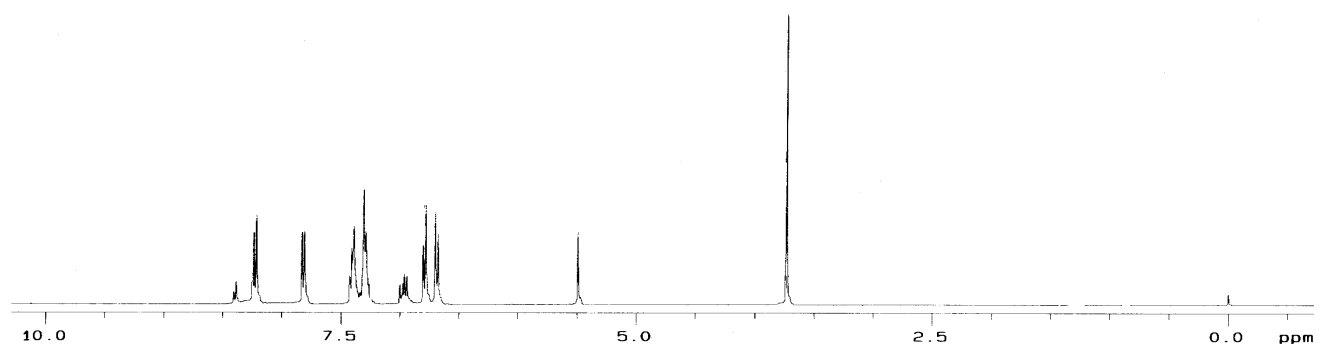

$400 \mathrm{MHz}{ }^{1} \mathrm{H}$ NMR Spectra for compound (Table 4, Entry 13).

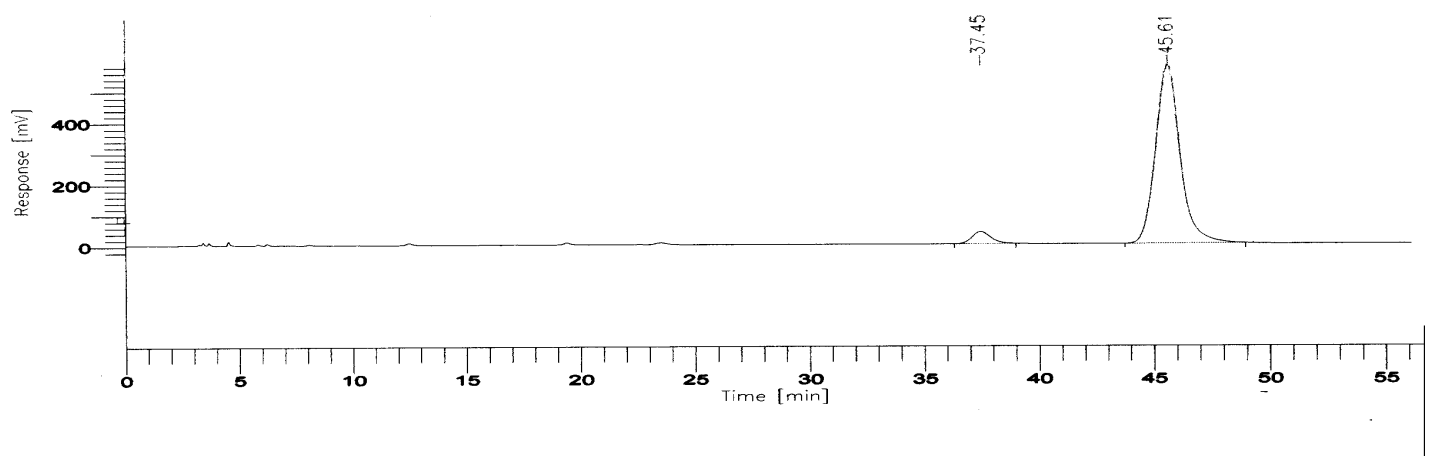

HPLC graph for racemic and enanrioenriched compound (Table 4, Entry 13).

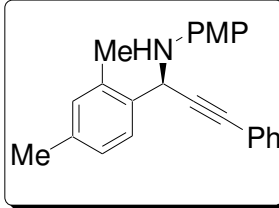

(R)-\{1-(2, 4-Dimethyl-phenyl)-3-phenyl-prop-2-ynyl\}-(4-methoxyphenyl)-amine (Table 4, Entry 14). $99 \%$ ee; isolated in $97 \%$ yield as yellow gel; $R_{\mathrm{f}}=$ 0.52 (EtOAc: petroleum ether = 1:9); HPLC conditions: Diacel chiralpak AD-H $(4.6 \mathrm{~cm}$ I.D. x $25 \mathrm{~cm}$ ), 80:20 hexanes: ${ }^{i}-\mathrm{PrOH}, 1.0 \mathrm{~mL} / \mathrm{min}$ flow rate, $\lambda=254 \mathrm{~nm}, \mathrm{~T}_{\mathrm{R}}$ (major) $=$ $9.90 \mathrm{~min}$ and $\mathrm{T}_{\mathrm{R}}$ (minor) $=8.88 \mathrm{~min}$; IR (thin film): 3368 (br), 2927, 2857, 1738, 1602, 1512, 1461, 1243, 1172, 1039, 841, 738, $699 \mathrm{~cm}-1 ;{ }^{1} \mathrm{H}$ NMR $\left(\mathrm{CDCl}_{3}, 400 \mathrm{MHz}\right): \delta 7.65$ $(\mathrm{d}, J=7.60 \mathrm{~Hz}, 1 \mathrm{H}), 7.38(\mathrm{~m}, 2 \mathrm{H}), 7.25(\mathrm{~m}, 3 \mathrm{H}), 7.05(\mathrm{~m}, 2 \mathrm{H}), 6.78(\mathrm{dd}, \mathrm{J}=32.04,9.04$ $\mathrm{Hz}, 4 \mathrm{H}), 5.44(\mathrm{~s}, 1 \mathrm{H}), 3.73(\mathrm{~s}, 3 \mathrm{H}), 2.42(\mathrm{~s}, 3 \mathrm{H}), 2.32$ (s, 3H); Anal. cacld. for $\mathrm{C}_{24} \mathrm{H}_{23} \mathrm{NO}$ : 
C, 84.42; H, 6.79; N, 4.10. Found: C, 84.70; H, 6.86; N, 4.19. Optical Rotation: $[\alpha]^{25}$ $+132.6\left(\mathrm{c} 1.25, \mathrm{CHCl}_{3}\right)$.

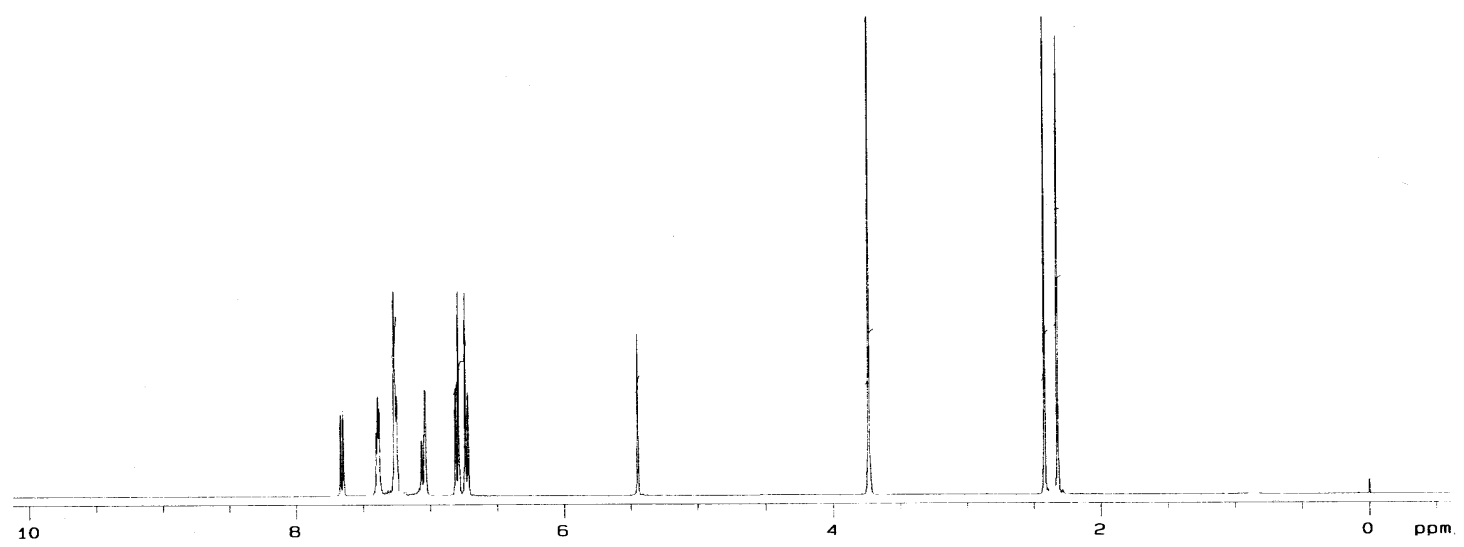

$400 \mathrm{MHz}{ }^{1} \mathrm{H}$ NMR Spectra for compound (Table 4, Entry 14).
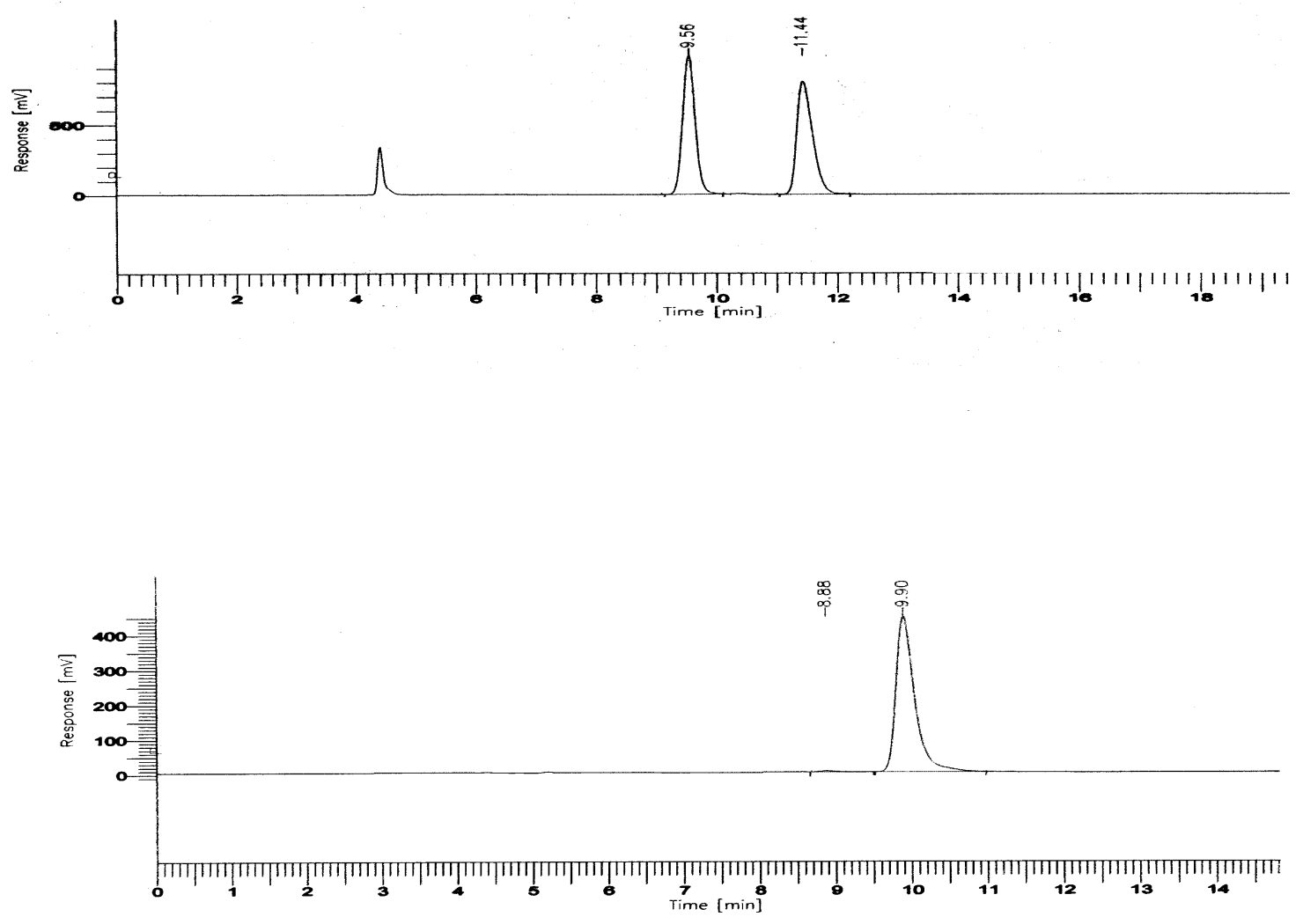

HPLC graph for racemic and enanrioenriched compound (Table 4, Entry 14). 


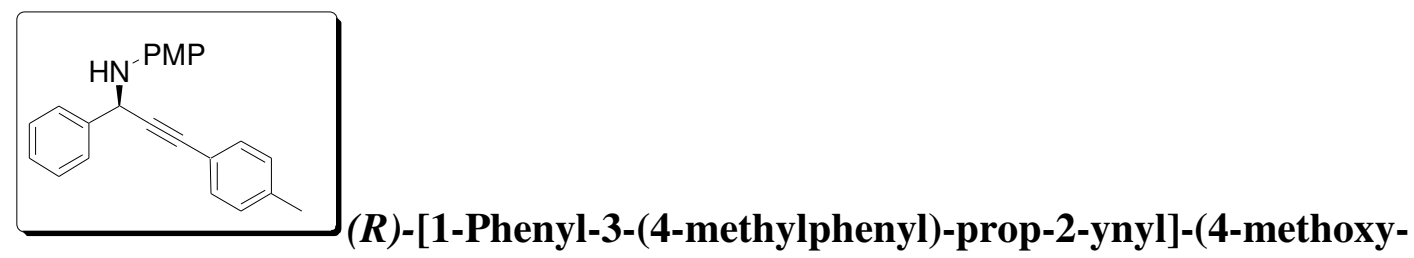

phenyl)-amine (Table 5, Entry 2). 93\% ee; isolated in 98\% yield as yellow gel; $\mathrm{R}_{\mathrm{f}}=$ 0.50 (EtOAc: petroleum ether = 1:9); HPLC conditions: Diacel chiralcel AD-H ( $4.6 \mathrm{~cm}$ I.D. $x 25 \mathrm{~cm}$ ), 80:20 hexanes: ${ }^{i}-\mathrm{PrOH}, 1.0 \mathrm{~mL} / \mathrm{min}$ flow rate, $\lambda=254 \mathrm{~nm}, \mathrm{~T}_{\mathrm{R}}$ (major) $=$ $13.62 \mathrm{~min}$ and $\mathrm{T}_{\mathrm{R}}$ (minor) $=11.37 \mathrm{~min} ;{ }^{1} \mathrm{H}$ NMR $\left(\mathrm{CDCl}_{3}, 400 \mathrm{MHz}\right): \delta 7.65(\mathrm{~d}, J=7.32$ $\mathrm{Hz}, 2 \mathrm{H}), 7.25-7.41(\mathrm{~m}, 5 \mathrm{H}), 7.08(\mathrm{~d}, J=8.04 \mathrm{~Hz}, 2 \mathrm{H}), 6.78(\mathrm{dd}, J=18.64,8.80 \mathrm{~Hz}, 4 \mathrm{H})$, 5.39 (s, $1 \mathrm{H}), 3.74$ (s, 3H), 2.33 (s, 3H); Anal. cacld. for $\mathrm{C}_{23} \mathrm{H}_{21} \mathrm{NO}$ : C, 84.37; H, 6.46; N, 4.28. Found: $\mathrm{C}, 84.63 ; \mathrm{H}, 6.63 ; \mathrm{N}, 4.47$. Optical Rotation: $[\alpha]_{\mathrm{D}}^{25}+123.7$ (c $\left.1.0, \mathrm{CHCl}_{3}\right)$.

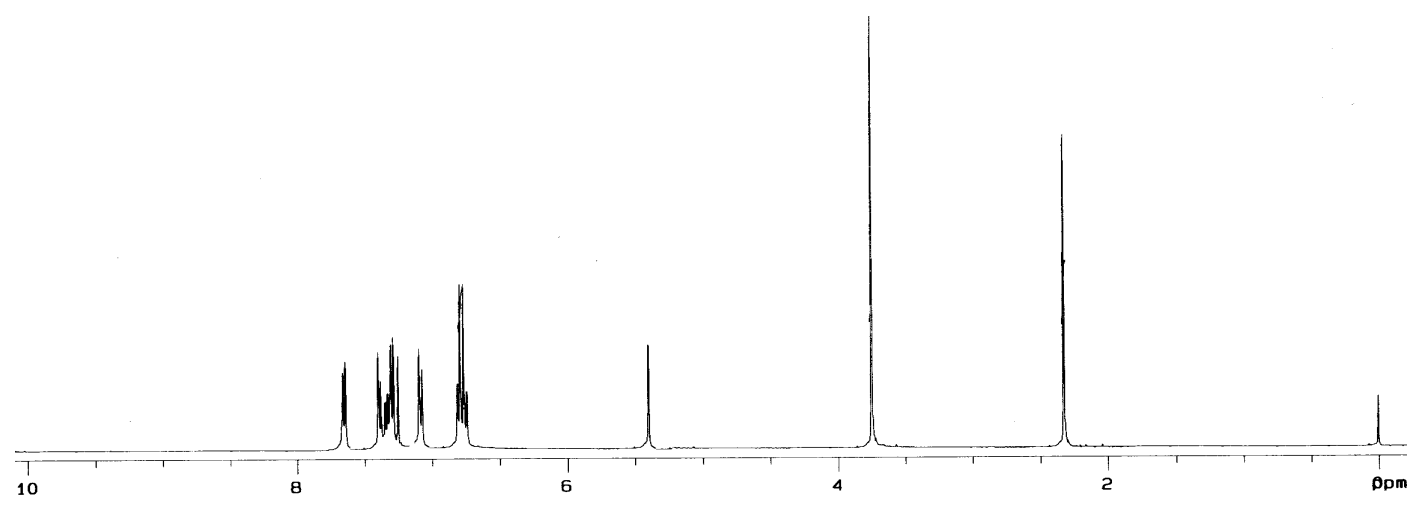

$400 \mathrm{MHz}{ }^{1} \mathrm{H}$ NMR Spectra for compound (Table 5, Entry 2).

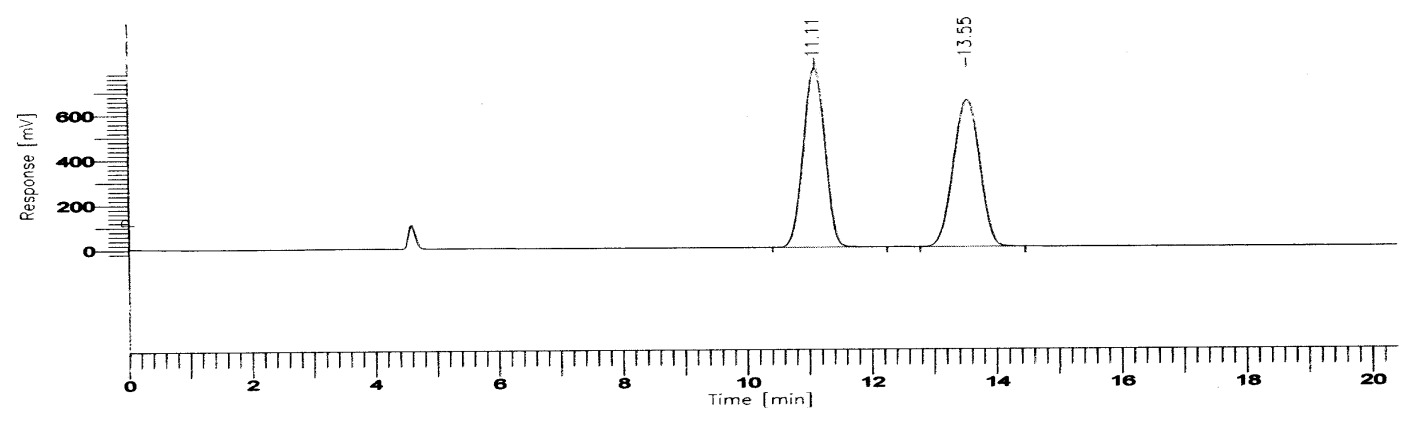




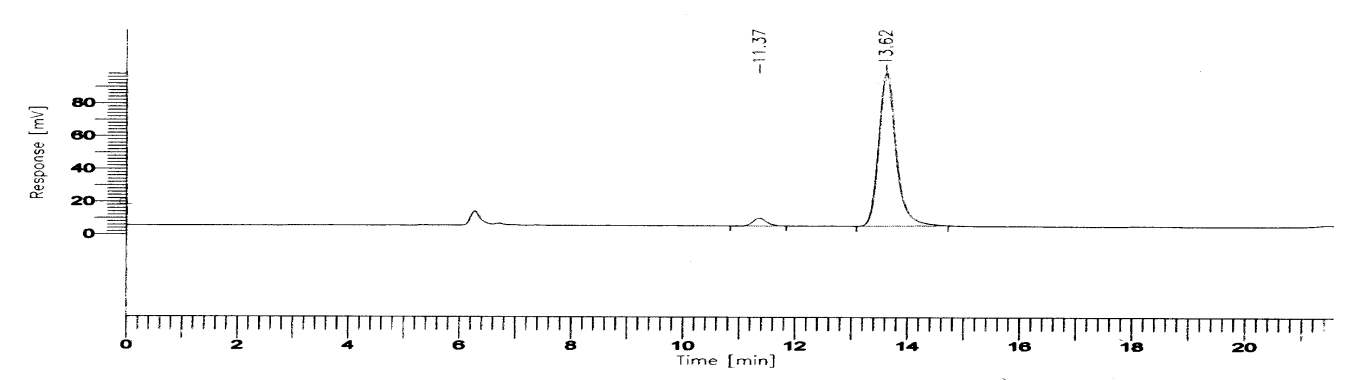

HPLC graph for racemic and enanrioenriched compound (Table 5, Entry 2).

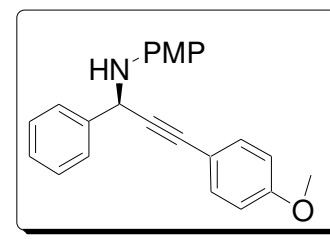

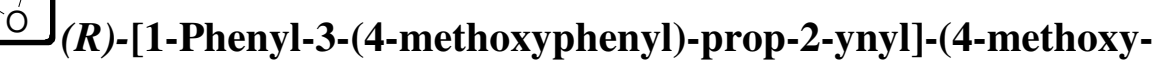

phenyl)-amine (Table 5, Entry 3). 93\% ee; isolated in $96 \%$ yield as yellow gel; $R_{\mathrm{f}}=$ 0.30 (EtOAc: petroleum ether = 1:9); HPLC conditions: Diacel chiralcel AD-H $(4.6 \mathrm{~cm}$ I.D. $x 25 \mathrm{~cm}$ ), 80:20 hexanes: ${ }^{i}-\mathrm{PrOH}, 1.0 \mathrm{~mL} / \mathrm{min}$ flow rate, $\lambda=254 \mathrm{~nm}, \mathrm{~T}_{\mathrm{R}}$ (major) $=$ $22.23 \mathrm{~min}$ and $\mathrm{T}_{\mathrm{R}}($ minor $)=18.10 \mathrm{~min} ;{ }^{1} \mathrm{H} \mathrm{NMR}\left(\mathrm{CDCl}_{3}, 400 \mathrm{MHz}\right): \delta 7.65(\mathrm{~d}, J=8.28$ $\mathrm{Hz}, 2 \mathrm{H}), 7.24-7.41$ (m, 5H), 6.73-6.81 (m, 6H), 5.39 (s, 1H), 3.78 (s, 3H), 3.74 (s, 3H); Anal. cacld. for $\mathrm{C}_{23} \mathrm{H}_{21} \mathrm{NO}_{2}$ : C, 80.44; H, 6.16; N, 4.08. Found: C, 80.67; H, 6.09; N, 4.09. Optical Rotation: $[\alpha]_{\mathrm{D}}^{25}+125.2\left(\mathrm{c} 1.0, \mathrm{CHCl}_{3}\right)$.

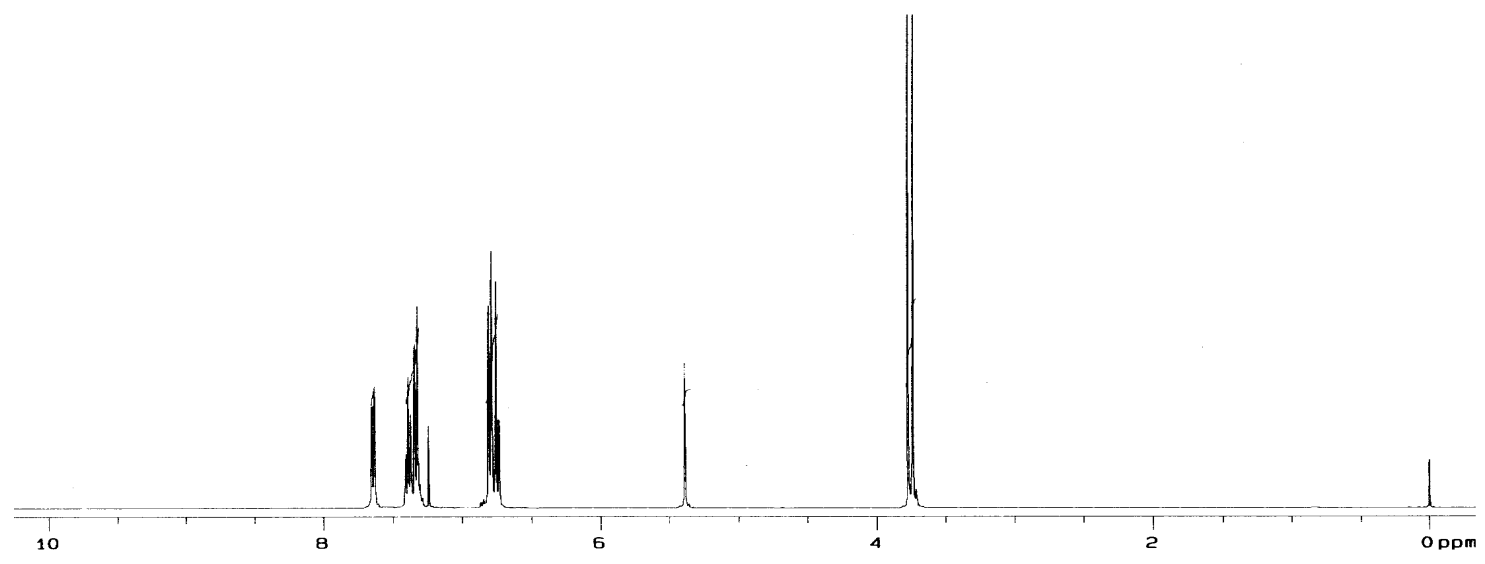

$400 \mathrm{MHz}{ }^{1} \mathrm{H}$ NMR Spectra for compound (Table 5, Entry 3). 

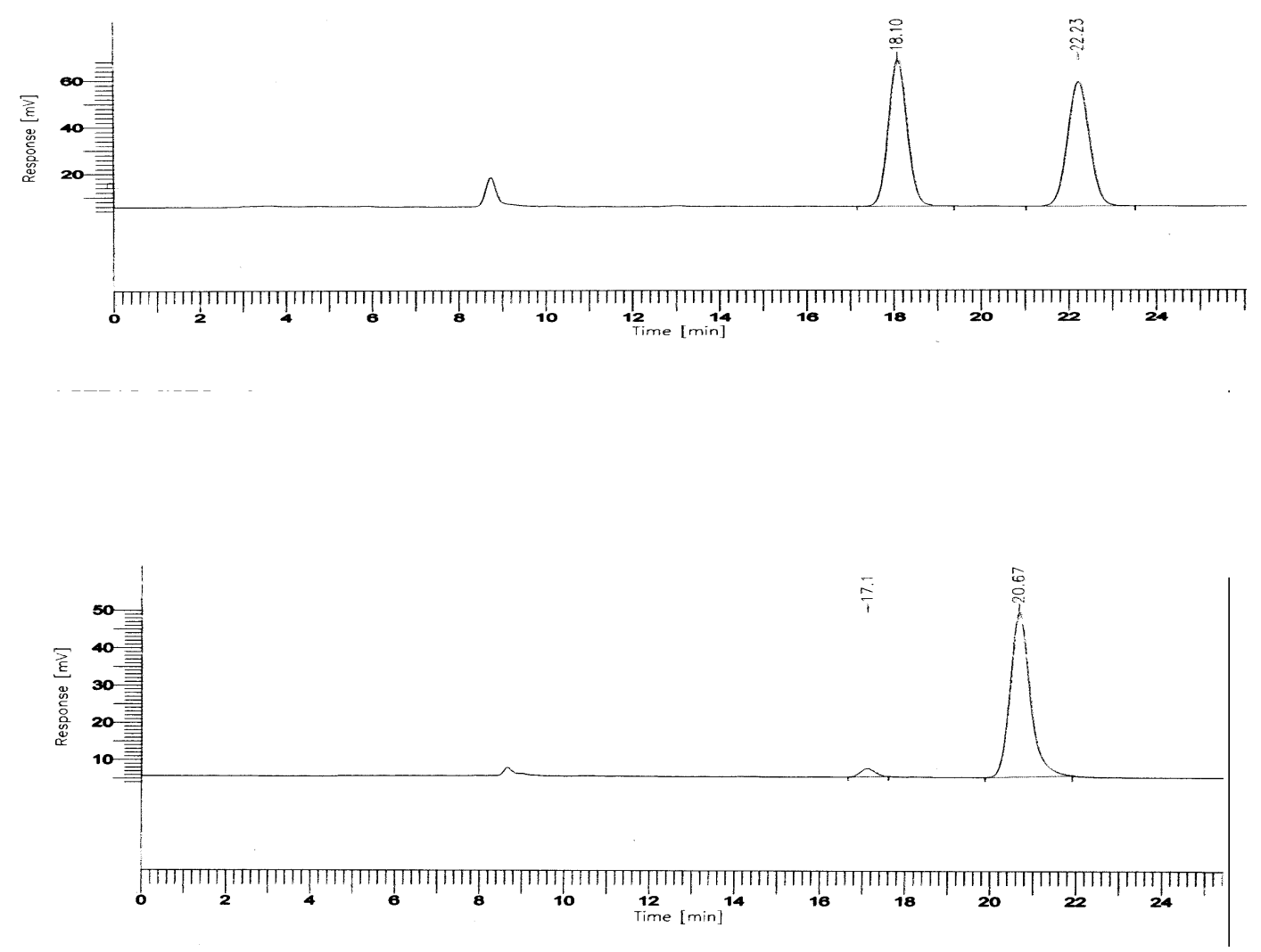

HPLC graph for racemic and enanrioenriched compound (Table 5, Entry 3).

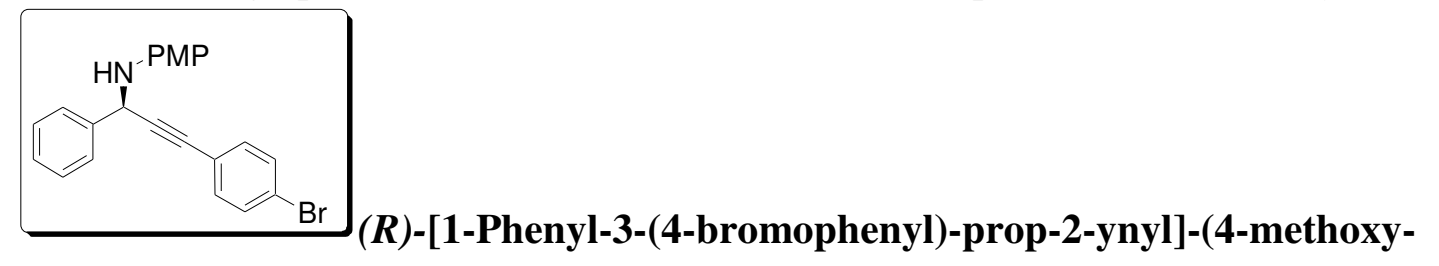

phenyl)-amine (Table 5, Entry 4). 90\% ee; isolated in 93\% yield as yellow gel; $R_{f}=$ 0.53 (EtOAc: petroleum ether = 1:9); HPLC conditions: Diacel chiralcel AD-H $(4.6 \mathrm{~cm}$ I.D. $x 25 \mathrm{~cm}$ ), 80:20 hexanes: ${ }^{i}-\mathrm{PrOH}, 1.0 \mathrm{~mL} / \mathrm{min}$ flow rate, $\lambda=254 \mathrm{~nm}, \mathrm{~T}_{\mathrm{R}}$ (major) $=$ $17.28 \mathrm{~min}$ and $\mathrm{T}_{\mathrm{R}}$ (minor) $=12.95 \mathrm{~min} ;{ }^{1} \mathrm{H} \mathrm{NMR}\left(\mathrm{CDCl}_{3}, 400 \mathrm{MHz}\right): \delta 7.64(\mathrm{~d}, J=7.32$ $\mathrm{Hz}, 2 \mathrm{H}), 7.38-7.46$ (m, 4H), 7.23-7.27 (m, 3H), 6.73-6.82 (m, 4H), 5.39 (s, 1H), 3.74 (s, $3 \mathrm{H}$ ); Anal. cacld. for $\mathrm{C}_{22} \mathrm{H}_{18} \mathrm{BrNO}$ : C, 67.36; H, 4.62; N, 3.57. Found: C, 67.69; H, 4.76; N, 3.60. Optical Rotation: $[\alpha]^{25}{ }_{\mathrm{D}}+89.9\left(\mathrm{c} 1.0, \mathrm{CHCl}_{3}\right)$. 


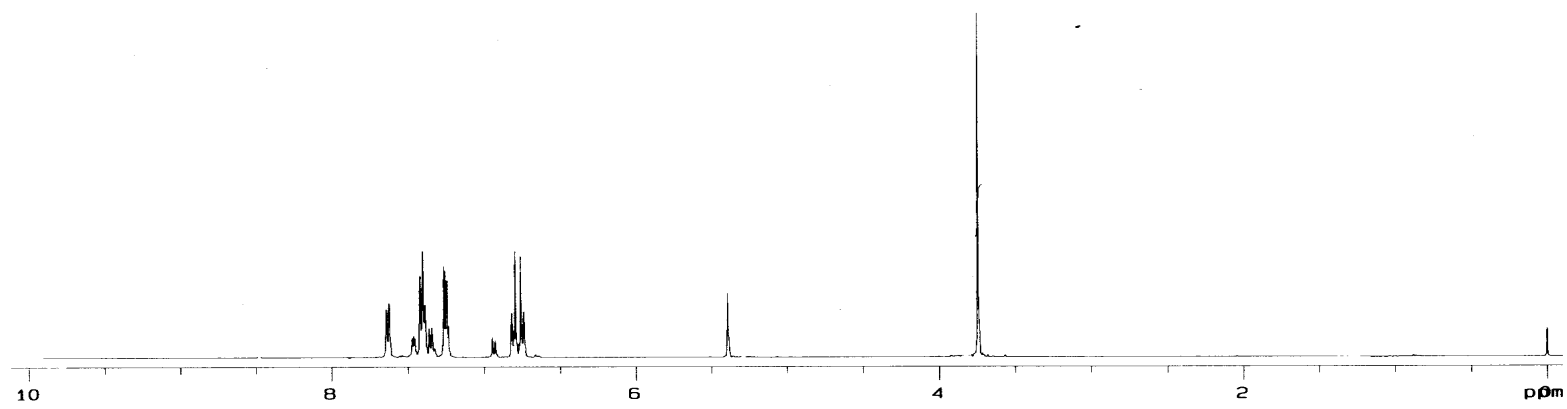

$400 \mathrm{MHz}{ }^{1} \mathrm{H}$ NMR Spectra for compound (Table 5, Entry 4).
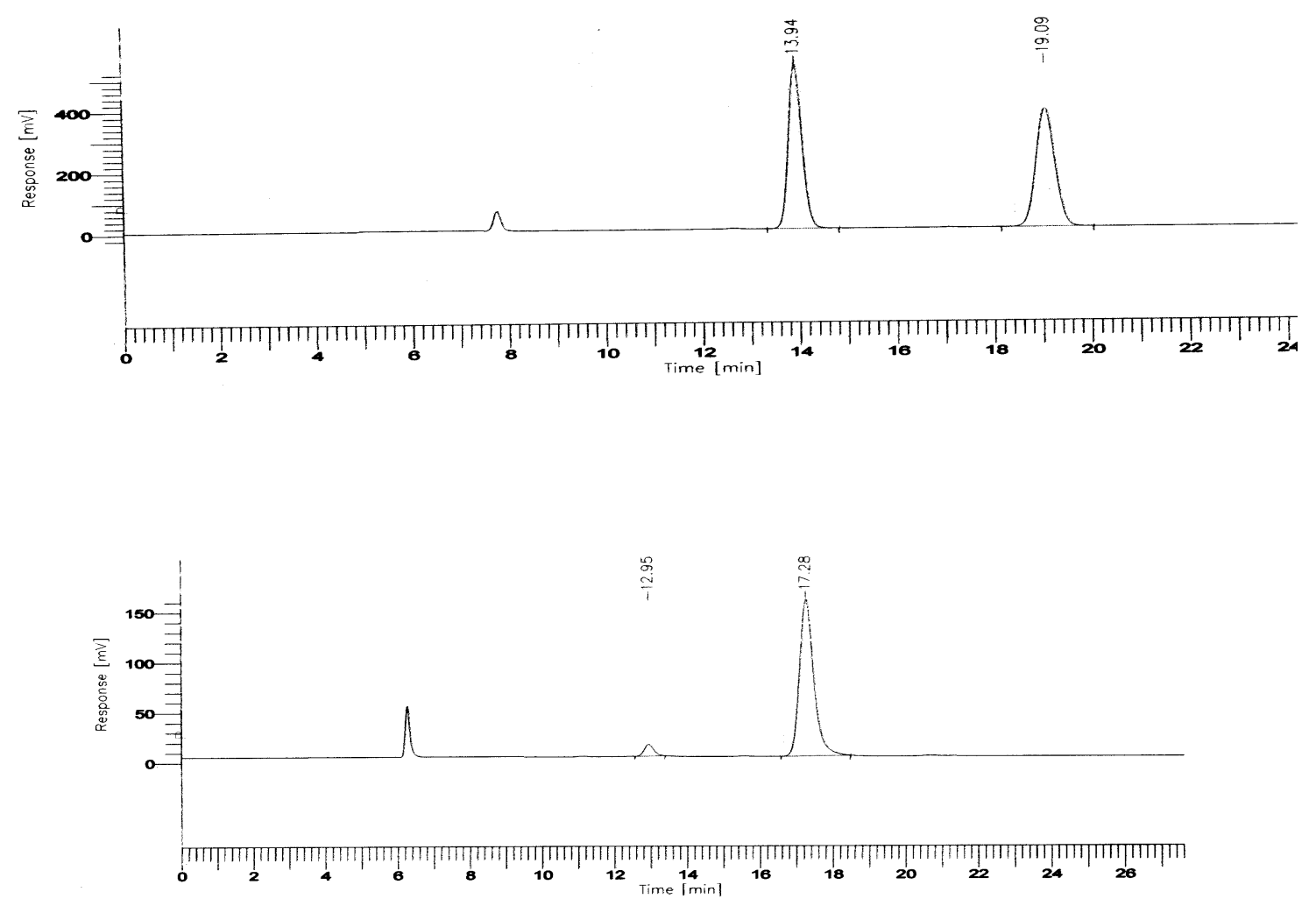

HPLC graph for racemic and enanrioenriched compound (Table 5, Entry 4). 


\section{References:}

1. C. Wei; C-J. Li J. Am. Chem. Soc. 2002, 124, 5638.

2. C. Wei; J. T. Mague; C-J. Li PNAS, 2004, 101, 5749.

3. C-J. Li; C. Wei Chem. Commun. 2002, 268.

4. S. Orlandi; F. Colombo; M. Benaglia Synthesis, 2005, 1689.

5. M. Benaglia; D. Negri; G. Dell'Anna Tetrahedron Lett. 2004, 45, 8705. 